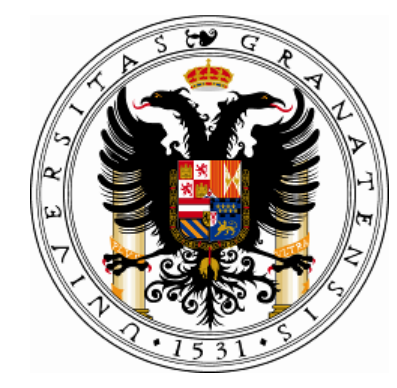

UNIVERSIDAD DE GRANADA

MÁSTER MÉTODOS Y TÉCNICAS AVANZADAS EN FÍSICA

TRABAJO FIN DE MÁSTER

Curso 2006-2007

\title{
SIMULACIÓN DE VIBRACIONES EN PLACAS DE MATERIAL COMPUESTO CFRP MEDIANTE EL MÉTODO DE ELEMENTOS FINITOS
}

PABLO MORENO GARCÍA

Directores:

ANTOLINO GALLEGO MOLINA

JOSE LUIS PÉREZ APARICIO 



\section{Índice general}

1. Introducción 1

2. Materiales compuestos $\quad 5$

2.1. Propiedades de los materiales compuestos . . . . . . . . . . . . 5

2.1.1. Introducción . . . . . . . . . . . . . . . . . . . . . . 5

2.1.2. Ecuaciones constitutivas . . . . . . . . . . . . . . . . . 9

2.1.3. Descripción bidimensional . . . . . . . . . . . . . . . . . 17

2.2. Cálculo de las frecuencias propias . . . . . . . . . . . . . . . . . . . . 19

2.3. Notación para placas multidireccionales . . . . . . . . . . . . . . . . 21

3. El método de los elementos finitos $\quad 23$

3.1. Historia del MEF . . . . . . . . . . . . . . . . . . . . 23

3.2. El Método de los Elementos Finitos (MEF) . . . . . . . . . . . . 25

3.2.1. Definición del Método . . . . . . . . . . . . . . . . . 25

3.2.2. Aplicación del Método . . . . . . . . . . . . . . . . . 25

3.2.3. Otros Detalles . . . . . . . . . . . . . . . . . 27

3.2.4. Funcionamiento de un Programa de Elementos Finitos . . . . . . . 28

3.2.5. Programas de Elementos Finitos utilizados . . . . . . . . . . . . . . 29

4. Placa unidireccional $\quad 31$

4.1. Cargas estáticas . . . . . . . . . . . . . . . . . . 31

4.1.1. Tracción . . . . . . . . . . . . . . . . . . 31

4.1.2. Compresión . . . . . . . . . . . . . . . . . . 32

4.1.3. Cortante . . . . . . . . . . . . . . . . . . 33

4.2. Cargas dinámicas . . . . . . . . . . . . . . . . . . . 35

4.2.1. Vibraciones naturales: Análisis modal . . . . . . . . . . . . . . 35

4.2.2. Vibracions forzadas: Análisis transitorio . . . . . . . . . . . . . . 44 
5. Placa multidireccional

5.1. Descripción de la placa y el experimento . . . . . . . . . . . . . . . 49

5.2. Resultados . . . . . . . . . . . . . . . . . . . . 52

5.2.1. Señales de vibración . . . . . . . . . . . . . . . . . 52

5.2.2. Frecuencias y modos propios . . . . . . . . . . . . . . . . . . 52 


\section{Índice de figuras}

2.1. Tensor de tensiones en una placa unidireccional cuando las coordenadas globales y locales coinciden con las direcciones ortótropas . . . . . . . . . . . . . . . . . . . . . . . 10

2.2. Rotación de los ejes de coordenadas desde un sistema de referencia global $1-2-3$ hasta un sistema local $x-y-z$. . . . . . . . . . . . . . . . . . . . . . . . . . . . . 13

2.3. Criterio de signos . . . . . . . . . . . . . . . . . . . . . . . . . . . . . . . . 17

2.4. Ejemplo de apilado definido según el código $\left[0_{3} / 90_{2} / 45 /-45_{3}\right]_{s}$. . . . . . . . . . . . 22

4.1. Ejemplo de placa [0] sometida a un esfuerzo estático de tracción . . . . . . . . . . . 32

4.2. Ejemplo de placa [0] sometida a un esfuerzo estático de compresión . . . . . . . . . . 33

4.3. Ejemplo de placa [0] sometida a un esfuerzo estático cortante . . . . . . . . . . . . . 34

4.4. Ejemplo de placa $[0]$ simplemente apoyada . . . . . . . . . . . . . . . . . . . . 35

4.5. Ejemplo de placa [0] empotrada . . . . . . . . . . . . . . . . . . . . . . . . . . . 37

4.6. Ejemplo de placa [0] empotrada-simplemente apoyada . . . . . . . . . . . . . . . . 38

4.7. Excitación y respuesta en dos puntos de una placa [0] simplemente apoyada en los dominios del tiempo y la frecuencia. Excitación: ruido gaussiano . . . . . . . . . . 45

4.8. Excitación y respuesta en dos puntos de una placa [0] simplemente apoyada en los dominios del tiempo y la frecuencia. Excitación: Pulso gaussiano . . . . . . . . . . . . 46

4.9. Excitación y respuesta en dos puntos de una placa [0] simplemente apoyada en los dominios del tiempo y la frecuencia. Excitación: Seno de $2.5 \mathrm{~Hz}$. . . . . . . . . . . . 47

4.10. Excitación y respuesta en dos puntos de una placa [0] simplemente apoyada en los dominios del tiempo y la frecuencia. Excitación: Seno de $40 \mathrm{~Hz}$. . . . . . . . . . . . 48

5.1. Esquema de la disposición de puntos en los que se colocaron los acelerómetros durante el experimento y dimensiones en cm . . . . . . . . . . . . . . . . . . . . . . . . . 50

5.2. Fotografía de la placa durante el experimento. Los acelerómetros se encuentran en la configuración 1. . . . . . . . . . . . . . . . . . . . . 51

5.3. (a) Señal de vibración registrada por el acelerómetro situado en el punto 53 durante el ensayo 1 (b) Transformada de Fourier de dicha señal . . . . . . . . . . . . . . . . . 53

5.4. Modos de vibración 1, 2, 3 y 4. Izquierda experimental, derecha numérico . . . . . . . 55 
5.5. Modo de vibración 5 , numérico . . . . . . . . . . . . . . . . 56

5.6. Modos de vibración 6 y 7. Izquierda experimental, derecha numérico . . . . . . . . 56

5.7. Modo de vibración 8. Izquierda experimental, derecha numérico . . . . . . . . . . . 57

5.8. Modos de vibración 9 y 10. Izquierda experimental, derecha numérico . . . . . . . . . 57

5.9. Modos de vibración 11 y 12. Izquierda experimental, derecha numérico . . . . . . . . 58

5.10. Modo de vibración 13. Izquierda experimental, derecha numérico . . . . . . . . . . 58

5.11. Modos de vibración 14 y 15. Izquierda experimental, derecha numérico . . . . . . . . 59

5.12. Modos de vibración 17 y 18. Izquierda experimental, derecha numérico . . . . . . . . 59

5.13. Modos de vibración 22 y 24. Izquierda experimental, derecha numérico . . . . . . . . 60 


\section{Indice de tablas}

2.1. Propiedades de las fibras . . . . . . . . . . . . . . . . . . . . . . 6

2.2. Propiedades de las matrices . . . . . . . . . . . . . . . . . . . . . . . . . . 7

2.3. Propiedades de diferentes materiales compuestos . . . . . . . . . . . . . . . . . . . 9

4.1. Deformación de una placa sometida a un esfuerzo estático de tracción. Comparación de los resultados teóricos y de simulación con FEAP . . . . . . . . . . . . . . . . . . 32

4.2. Deformación de una placa sometida a un esfuerzo estático de compresión. Comparación de los resultados teóricos y de simulación con FEAP . . . . . . . . . . . . . . . 33

4.3. Deformación de una placa sometida a un esfuerzo estático cortante. Comparación de los resultados teóricos y de simulación con FEAP ～. . . . . . . . . . . . . . . . . . 34

4.4. Frecuencias propias de una placa [0] simplemente apoyada obtenidas teóricamente y con los programas FEAP y Ansys, todos los valores en $\mathrm{Hz}$. . . . . . . . . . . . 36

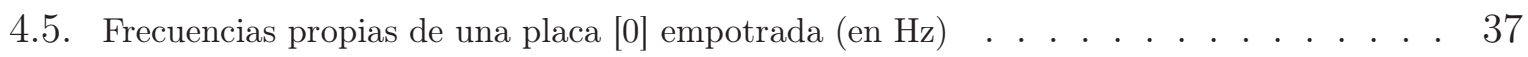

4.6. Frecuencias propias de una placa [0] empotrada-simplemente apoyada (en $\mathrm{Hz}$ ) . . . . . 38

4.7. Frecuencias propias de una placa cuadrada empotrada con fibras a diferentes ángulos $(\mathrm{en} \mathrm{Hz}) \quad \ldots \ldots \ldots$

4.8. Frecuencias propias de una placa rectangular empotrada con fibras a diferentes ángulos

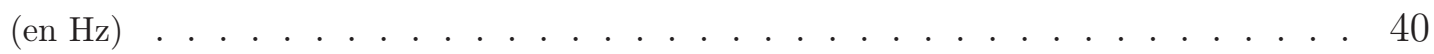

4.9. Modos propios y frecuencias propias (en $\mathrm{Hz}$ ) de una placa [90] cuadrada empotrada . . 42

4.10. Modos propios y frecuencias propias (en $\mathrm{Hz}$ ) de una placa [45] cuadrada empotrada . . 43

5.1. Disposición de los acelerómetros en cada una de las 17 configuraciones . . . . . . . 51

5.2. Frecuencias propias de la placa obtenidas en cada uno de los ensayos y su media, en $\mathrm{Hz} \quad 54$

5.3. Frecuencias propias de la placa obtenidas de forma experimental y numérica, en $\mathrm{Hz} \quad$. . 54 



\section{Capítulo 1}

\section{Introducción}

Los materiales compuestos son hoy día muy usados en diversos sectores industriales. $\mathrm{Su}$ baja densidad y alta resistencia y rigidez, son propiedades físicas que les han permitido su introducción en sectores tan exigentes como el aeroespacial. Entre ellos, unos de los más usados son los materiales CFRP (Polímeros Reforzados con Fibra de Carbono). Sin embargo, es bien conocido que sus propiedades mecánicas pueden verse considerablemente degradadas en presencia de daño. En sectores como el de la construcción aeroespacial, el tamaño de fallo crítico admitido es considerablemente pequeño.

Debido a su construcción multicapa, el daño más habitual y que más peligro potencial proporciona, consiste en una delaminación interna, que además frecuentemente se produce tras un impacto de un objeto externo o en el proceso de manufactura [4]. Esto introduce cambios en la rigidez y amortiguamiento de la estructura fabricada con dicho material, causando variaciones en sus respuesta vibracional (una variación en sus resonancias naturales, coeficientes de amortiguamiento y modos propios), que pueden ser usados como indicadores de daño. Estos cambios son en general muy pequeños y afectan sobre todo a las resonancias naturales de alto orden, dejando casi intactas las resonancias de baja frecuencia. Los cambios en las resonancias de baja frecuencia se hacen más importantes a medida que el tamaño de fallo crece.

Por motivos de seguridad y bajos costes, es conveniente desarrollar sistemas de diagnóstico poco invasivos, automáticos y que permitan hacer un diagnóstico espaciotemporal extenso de la estructura, y permitan detectar daño en etapas tempranas. El reto es desarrollar un sistema con una alta capacidad de discriminación entre estructuras intactas y dañadas, que permita la detección temprana del daño, para prevenir su deterioro o fallo catastrófico. 
Este aspecto ha sido objeto de estudio de muchas investigaciones, desarrollándose múltiples técnicas de lo que genéricamente se denominan "Ensayos No Destructivos (NDT)", como Ensayos de Vibraciones, Ultrasonidos, Emisión Acústica, Termografía, etc. Todas ellas inspeccionan a la estructura en intervalos de tiempo y posiciones fijas, es decir, cuando los técnicos lo realizan. Se denominan "métodos locales". En contraste, durante la última década ha tomado mucha fuerza la idea de inspeccionar la "salud" de toda la estructura de manera continuada en el tiempo, extrayendo características de la misma que permitan aportar permanentemente conclusiones sobre su estado, y por tanto, sobre su tiempo de vida y la necesidad de eventuales reparaciones ("métodos globales"). Esta idea constituye una disciplina propia de trabajo $\mathrm{I}+\mathrm{D}+\mathrm{I}$, denominada "Structural Health Monitoring (SHM)". Véase el trabajo de Chang [3] donde se indican varios aspectos científicos, económicos y aplicaciones del SHM. De acuerdo con Rytter [10], existen 4 niveles de diagnóstico de daño estructural: Nivel 1, detección de la existencia de daño, Nivel 2, localización del daño, Nivel 3: extensión del daño y Nivel 4, pronóstico del tiempo de vida restante de la estructura.

Existen varios procedimientos para abordar la detección de daño (Nivel 1), dependiendo del tipo de estructura. Quizás los más desarrollados son los que se basan en extraer los modos propios de vibración de la estructura (análisis modal), intentando observar variaciones de los mismos cuando la estructura está dañada [9]. No obstante, cuando el tamaño del defecto es pequeño, estos procedimientos con los tratamientos de señal que habitualmente se usan, no llegan a tener la suficiente capacidad de discriminación. Es por ello que recientemente están teniendo más auge los procedimientos en el análisis de la respuesta de vibración transitoria de la estructura en el dominio del tiempo [6]. No obstante, el desarrollo actual de estas técnicas no es el mismo que el de las primeras, fundamentalmente por la escasez de algoritmos capaces de correlacionar la respuesta experimental de la estructura con su daño y llevar esta información a una forma útil, sin falsas alarmas.

En definitiva, para aumentar la sensibilidad a la existencia del defecto, e incluso para mejorar su ubicación (Nivel 2), se requiere introducir modelos computacionales [3], [6]. Además, tal y como se indica en [6], se hace necesaria la participación en el problema de técnicas avanzadas de tratamiento de señales que permitan aumentar la capacidad de discriminación entre estructuras dañadas y no dañadas, a partir de un análisis más productivo de los datos captados por los sensores. Debido a la composición del material, es difícil establecer modelos analíticos precisos, por lo que el modelo numérico elegido juega un importante papel en la detección del daño.

Este Trabajo de Investigación Tutelada (TIT) constituye la fase inicial del trabajo predoctoral a desarrollar dentro de una beca FPI (Formación del Personal Investigador) asociada al proyecto del Plan Nacional Diseño y Producción Industrial titulado "Aplicación 
de técnicas avanzadas de tratamiento de señales a la detección y caracterización de daño (acrónimo SEDA; código DPI2006-02970", Dirigido por el Dr. Gallego, cuyos objetivos son los siguientes:

1. Simular numéricamente mediante el Método de los Elementos Finitos (MEF) las vibraciones de una placa de material compuesto CFRP intacta y con una delaminación interna.

2. Desarrollar algoritmos basados en modelos ARMA y la Transformada Wavelet para la discriminación entre placas CFRP dañadas y no dañadas (con una delaminación interna) a partir de señales transitorias de vibración.

3. Verificar y comparar los algoritmos anteriores con datos de simulación y experimentales. Se realizarán experimentos de vibraciones con sensores y actuadores piezoelectricos tipo PZT, más ligeros y adaptables a la estructura que los convencionales acelerómetros.

Como primera fase de la etapa de simulación numérica con el MEF, en este TIT se ha validado un modelo de elementos finitos para placas sin daño (intactas). Se ha trabajado con los programas comerciales FEAP y Ansys. El programa FEAP, a cuyo código fuente se tiene acceso y sobre el que se pueden programar otras aplicaciones o variantes, tiene desarrollados elementos finitos solo para placas unidireccionales. Sin embargo, el programa Ansys, a cuyo código fuente no se puede acceder y por tanto no permite la realización de ampliaciones, tiene disponible la posibilidad de simular placas multidireccionales. Como el objetivo en la futura tesis doctoral, tal y como se ha comentado anteriormente, es analizar placas con una delaminación interna, es necesario modelar un nuevo elemento finito válido para este tipo de placas, por lo que no será posible trabajar con el programa Ansys, sino con FEAP. Esta es la razón por la que en esta primera fase se ha trabajado con los dos códigos.

La validación se ha realizado sobre dos tipos de placas:

- Placas unidireccionales (Capítulo 4): Validación de los programas FEAP y Ansys, comparándolos con los resultados teóricos. En este caso se han realizado las siguientes tareas:

- Se han aplicado cargas estáticas de tracción, compresión y cortante, comparando los deformaciones de la placa obtenidas con el modelo numérico y las predichas por las ecuaciones constitutivas de la elasticidad lineal, a partir de las propiedades de la placa. 
- Se han obtenido las frecuencias y modos propios de diferentes tipos de placas según la orientación de sus fibras y sus condiciones de contorno. Se han comparado con los resultados teóricos.

- Se ha obtenido la respuesta temporal de la placa en cualquier punto de la misma, excitándola en un punto arbitrario, con diferentes tipos de excitación: senoidal, tipo ruido blanco y tipo pulso gausiano. En este caso la validación se ha realizado en el dominio de la frecuencia (comparando con las frecuencias propias de vibración teóricas) pues no se dispone de solución analítica en el dominio del tiempo.

- Placas multidireccionales (Capítulo 5): Validación del programa Ansys, comparando los resultados de simulación con los obtenidos experimentalmente en un ensayos de vibración por martillo de impacto realizados en el proyecto del Plan Nacional DPI "Diagnóstico de daño en estructuras de alta responsabilidad hechas de material compuesto (acrónimo DIDA; código DPI2002-04472-C02-02)", dirigido por el Dr. Pérez-Aparicio y coordinado con el Dr. Güemes de la Escuela de Ingenierios Aeronáuticos de la UPM, y en el que ha participado el Dr. Gallego. En este caso se han comparado las frecuencias de resonancia experimentales y simuladas, así como los modos de vibración obtenidos mediante simulación y los obtenidos mediante el programa comercial Artemis, a partir de las señales de vibración recogidas en 85 puntos de la placa.

La Memoria se completa con los Capítulos 2 y 3 dedicados a propiedades elásticas de los materiales compuestos, y a una introducción al Método de Elementos Finitos y los programas FEAP y Ansys, respectivamente. 


\section{Capítulo 2}

\section{Materiales compuestos}

\subsection{Propiedades de los materiales compuestos}

Esta sección tiene como finalidad exponer las propiedades fundamentales de los materiales compuestos. En primer lugar comentaremos las propiedades generales de estos materiales así como su proceso de fabricación para pasar posteriormente a analizar las ecuaciones que rigen su comportamiento caracterizado principalmente por su anisotropía.

\subsubsection{Introducción}

Los materiales compuestos son ideales para aplicaciones donde se requiere una buena relación resistencia-peso y rigidez-peso. Su estudio comprende muchas áreas como los procesos de fabricación, la elasticidad anisótropa, la resistencia de materiales anisótropos y la micromecánica.

La palabra compuesto significa que dos o más materiales son combinados a escala macroscópica para formar un nuevo material de mejores características. Los materiales compuestos pueden presentar varias estructuras, por un lado podemos tener un material formado por la superposición de placas de varios materiales, y por otro se puede conseguir un material compuesto agregando partículas de uno o varios materiales en una matriz. También podemos formar el material compuesto mediante fibras dentro de una matriz. Este último caso, de escala macroscópica, es el tipo de materiales compuestos que hemos estudiado en este trabajo [8].

Existen algunos materiales que presentan mayor rigidez y resistencia a la rotura en la forma de fibras largas que en otras formas de mayor volumen. Por ejemplo, un cristal de vidrio es mucho más débil que las fibras del mismo material. La geometría es obviamente un factor crucial en las propiedades del material, debido a que en su forma de fibra la estructura del vidrio es más perfecta, con un mejor alineamiento de moléculas y menos 
defectos en su malla. Una fibra está caracterizada geométricamente no sólo por su relación longitud-radio sino también por el diámetro de las fibras, habitualmente cercano al tamaño de la malla de cristalización. Las fibras utilizadas en materiales compuestos tienen un diámetro de alrededor de $10(\mu m)$. La razón de este tamaño óptimo tan pequeño fue descubierto por Griffith (1921) cuando experimentó que probetas de vidrio de diferente diámetro tenían resistencias muy diferentes.

Las propiedades típicas que presentan las fibras son elevada resistencia mecánica, flexibilidad, ligereza de peso y facilidad de proceso. Las fibras más habituales son de vidrio, de carbono y de aramida.

El vidrio es un material amorfo obtenido en un proceso de enfriamiento rápido que evita la cristalización en forma de cuarzo pues su componente principal es Silicio. La fibra de vidrio en comparación con el resto de las fibras se caracteriza por su bajo coste, buena resistencia a tracción y a agentes químicos, buen comportamiento como aislante eléctrico, baja rigidez, propensión a sufrir daño superficial, baja resistencia a fatiga y alta dureza, lo que conlleva un desgaste elevado del utillaje. La fibra de vidrio se clasifica en tres grupos. El E-vidrio es utilizado en aplicaciones eléctricas y generales debido a que es un buen aislante eléctrico. Además tiene alta durabilidad, su coste es bajo y absorbe poco la humedad. El A-vidrio presenta elevada resistencia en medios alcalinos aunque posee propiedades mecánicas menores. El tercer tipo es el S-vidrio que se caracteriza por su elevada resistencia.

Las fibras de carbono contienen una mezcla de carbón amorfo y grafito. Respecto a otras fibras la fibra de carbono tiene un peso bajo, presenta una rigidez y resistencia altas, tanto absolutas como específicas, su coeficiente de expansión lineal es muy bajo y su resistencia a fatiga alta. Como desventajas encontramos su alto coste, baja resistencia a impactos y su alta conductividad eléctrica.

Un ejemplo de aramida (poliamida aromática) es la K49 que tiene la densidad específica mínima y la resistencia específica más alta de cualquier fibra comercial. Otras fibras pueden ser la HM-50 (aramida copolimérica) o la Spectra (polietileno).

\begin{tabular}{|c|c|c|c|c|c|}
\hline & E-vidrio & A-vidrio & S-vidrio & Carbono & Aramida \\
\hline \hline Densidad $\left(\mathrm{g} / \mathrm{cm}^{3}\right)$ & 2.58 & 2.50 & 2.48 & 1.75 & 1.44 \\
\hline Resistencia $(\mathrm{GPa})$ & 3.50 & 3.00 & 4.60 & 3.31 & 2.80 \\
\hline Rigidez $(\mathrm{GPa})$ & 72.5 & 69.0 & 86.0 & 228.0 & 133.0 \\
\hline Coef. de dilat. térmica $\left(10^{-6} / \mathrm{K}\right)$ & 5.0 & 8.6 & 5.6 & $-0.7 / 9.0$ & $-2.0 / 60.0$ \\
\hline
\end{tabular}

Tabla 2.1: Propiedades de las fibras

La matriz es el material que rodea, protege y soporta a las fibras para transferir tensiones entre ellas, protegerlas de las condiciones ambientales, proteger su superficie contra 
el desgaste y prevenir su pandeo. Las propiedades de la matriz afectan poco a las propiedades mecánicas a tracción, bastante a las propiedades mecánicas a cortante, mucho a las propiedades mecánicas a compresión y también deberemos tenerla en cuenta al estudiar la fabricabilidad (viscosidad, punto de fusión y temperatura de curado). Los materiales más utilizados para la fabricación de las matrices serán los materiales termoestables (epoxy, poliester, vinilester, fenólica y poliamidas). Este tipo de material se caracteriza por su viscosidad muy baja antes del curado, su estabilidad térmica y resistencia química, su poca fluencia y relajación por tensión, su facilidad de fabricación, su buena capacidad de preimpregnado y su bajo coste.

El epoxy es una resina líquida de bajo peso orgánico que contiene grupos epóxidos. El curado se hace mediante polimerizado y la estructura molecular resultante es sólida e irreversible. Sus ventajas son la ausencia de volátiles y baja contracción durante el curado, la estabilidad contra agentes químicos y disolventes y la adhesión a casi todas las fibras. Sus inconvenientes principales son su elevado coste y el largo tiempo de curado. El poliester es un compuesto con enlaces de carbono dobles diluidos en un reactivo (estireno) que presenta una reacción química de curado mediante catalizador. Sus propiedades son la baja viscosidad, el tiempo de curado rápido y el bajo coste, además de la alta contracción durante el curado y las propiedades mecánicas inferiores al epoxy. Químicamente el vinilester se define como cadenas de moléculas epoxy con dobles enlaces en los finales. Su proceso de curado es similar al del poliester. Presenta estabilidad contra agentes químicos y disolventes, buenas propiedades mecánicas, baja viscosidad, tiempo de curado rápido y buena adhesibilidad a las fibras aunque sufre una alta contracción durante el curado.

\begin{tabular}{|c|c|c|c|c|c|c|}
\hline & $\begin{array}{c}\text { Coef. de dilatación } \\
\text { térmica }\left(\frac{10^{-6} \mathrm{~mm}}{{ }^{0} \mathrm{C}}\right)\end{array}$ & $\begin{array}{c}\text { Densidad } \\
\left(\frac{\mathrm{g}}{\mathrm{cm}^{3}}\right)\end{array}$ & $\begin{array}{c}\text { Resistencia } \\
(M P a)\end{array}$ & $\begin{array}{c}\text { Rigidez } \\
(G P a)\end{array}$ & $\begin{array}{c}\text { Elong. } \\
(\%)\end{array}$ & $\begin{array}{c}\text { Curado } \\
(\%)\end{array}$ \\
\hline \hline Epoxy & $50-80$ & $1.2-1.3$ & $55-130$ & $2.7-4.1$ & $2.0-3.2$ & $1.0-5.0$ \\
\hline Poliester & $60-205$ & $1.1-1.4$ & $35-103$ & $2.1-4.1$ & $1.0-5.0$ & $5.0-12.0$ \\
\hline Vinilester & $93-135$ & $1.1-1.3$ & $73-81$ & $3.0-3.5$ & $3.5-5.5$ & $5.4-10.3$ \\
\hline
\end{tabular}

Tabla 2.2: Propiedades de las matrices

Los materiales compuestos presentan unas características que los hacen diferentes de los materiales más convencionales en ingeniería y que condicionan la acometida de proyectos en los que se presenten estos materiales. Estas son su falta de homogeneidad y de isotropía (nos encontramos ante materiales ortótropos y en general anisótropos). Esto hace que estos materiales requieran herramientas analíticas y experimentales especiales.

Una placa es la forma más sencilla en la que se puede presentar un material compuesto. Una placa es una placa con fibras alineadas en una dirección dentro de una matriz de otro material. Las fibras son los elementos que aguantan la carga sobre la placa. La principal 
función de la matriz es soportar las fibras y distribuir la carga entre todas ellas. La última función de la matriz es, en caso de rotura de alguna de las fibras, transferir carga desde un extremo de esta fibra rota al otro. El comportamiento de las fibras es claramente elástico lineal, aunque las matrices de materiales resinosos, el caso más habitual, presentan un comportamiento más complejo, pudiendo ser viscoelástico o viscoplástico. Aún así a los materiales compuestos que estudiaremos les asignaremos un comportamiento lineal elástico, pues la mayor parte de su rigidez y resistencia se debe a las fibras.

Por otro lado tenemos el laminado. El laminado es un apilado de varias placas que pueden presentar diferentes direcciones de fibras. Las capas de un laminado están pegadas por el mismo material de la matriz. Normalmente el motivo del apilado en diferentes direcciones de las placas es ajustar las propiedades de la estructura con los requerimientos de carga de cada caso particular.

El proceso de fabricación de los materiales compuestos condiciona en gran parte su uso posterior. Este proceso consta de dos pasos fundamentales, el apilado y el curado. El apilado es el ordenado de las fibras en placas y de las placas en capas o laminados. El curado es el secado o polimerización de la matriz resinosa para formar una unión permanente entre las fibras y las placas. El curado puede ocurrir sin ayuda o puede consistir en aplicar calor y/o presión para acelerar el proceso de polimerización. Pasos secundarios en el proceso de fabricación son el pegado después del curado, el pintado, el mecanizado y el ensamblaje. Hay tres procesos de apilado principales para los materiales compuestos reforzados mediante fibras: el enrollado continuo y apilado, el moldeo y la laminación continua. La elección del proceso se hará tras analizar el coste, la efectividad del proceso, la experiencia en las diferentes técnicas, etc.

El proceso de enrollamiento continuo y apilado de capas consta de tres partes. Primero las fibras pasan a través de la resina líquida y son enrolladas en un útil. Las fibras son entonces orientadas con diferentes direcciones sobre el útil. La pieza completa es curada sobre el útil después de lo cual éste es separado. El proceso de apilado por capas comienza con capas de fibras preimpegnadas que se mantienen unidas mediante una material separable que sirve de apoyo. Las capas se colocan de acuerdo a la forma y dirección de fibras deseadas.

El moldeo es una operación de menor importancia. El proceso comienza con la colocación manual o automática de las fibras preimpregnadas en capas. Posteriormente las capas son comprimidas a elevadas temperaturas para formar el laminado final. El moldeo se emplea, por ejemplo, para fabricar cúpulas de radar con pequeñas tolerancias en espesor. La laminación continua implica la unión de placas mediante presión por rodillos. De esta forma se fabrican elementos para la construcción de estructuras. Este proceso es conocido también como pultrusión o tractrusión. 
Por lo expuesto los materiales compuestos presentan numerosas ventajas frente a los materiales convencionales. Las principales ventajas de estos materiales son la ligereza, su resistencia a la corrosión y al daño por fatiga, su menor gasto de mecanizado (las piezas de materiales compuestos no son normalmente mecanizadas, salen directamente de molde), la menor necesidad de elementos de unión (estos materiales no se suelen soldar, roblonar ni atornillar, por lo que las piezas se hacen enteras), la capacidad de optimizar la rigidez y resistencia mediante el diseño, etc. También cabe reseñar las desventajas que presenta el material compuesto para tenerlas en cuenta en el proceso de diseño. Las principales son su elevado coste debido a su baja producción, la falta de normativa y experiencia en el diseño, la degradación por agentes ambientales, su pobre capacidad de absorber impactos en general y la dificultad de inspección no destructiva.

\begin{tabular}{|c|c|c|c|c|c|}
\hline Fibra & T300 & B(4) & AS & E-glass & Kev 49 \\
\hline Matriz & N5208 & N5505 & 3501 & Epoxy & Epoxy \\
\hline$E_{x x}(G P a)$ & 181 & 204 & 138 & 38.6 & 76 \\
\hline$E_{y y}(G P a)$ & 10.3 & 18.5 & 8.96 & 8.27 & 5.5 \\
\hline$\nu_{x}$ & 0.28 & 0.23 & 0.30 & 0.26 & 0.34 \\
\hline$E_{s}(G P a)$ & 7.17 & 5.59 & 7.1 & 4.14 & 2.3 \\
\hline$\nu_{f}$ & 0.70 & 0.50 & 0.66 & 0.45 & 0.60 \\
\hline$\rho\left(\mathrm{g} / \mathrm{cm}^{3}\right)$ & 1.6 & 2.0 & 1.6 & 1.8 & 1.46 \\
\hline
\end{tabular}

Tabla 2.3: Propiedades de diferentes materiales compuestos

\subsubsection{Ecuaciones constitutivas}

\section{Rigidez de los materiales compuestos unidireccionales}

En esta sección desarrollamos las ecuaciones constitutivas de los materiales compuestos cuando el sistema de referencia local orientado según la dirección de la fibras y el sistema de referencia global según la dirección de la placa coinciden [1], véase la Figura 2.1. Como es bien conocido, los tensores tensión y deformación son simétricos: sólo se consideran seis componentes por lo que los tensores se representan mediante vectores y se usa la siguiente notación para la tensión y la deformación, respectivamente:

$$
\begin{aligned}
& \{\sigma\}=\left\{\sigma_{1}, \sigma_{2}, \sigma_{3}, \tau_{23}, \tau_{13}, \tau_{12}\right\}^{T}, \\
& \{\epsilon\}=\left\{\epsilon_{1}, \epsilon_{2}, \epsilon_{3}, \gamma_{23}, \gamma_{13}, \gamma_{12}\right\}^{T},
\end{aligned}
$$

lo que corresponde en notación contraída con: 


$$
\begin{gathered}
\{\sigma\}=\left\{\sigma_{1}, \sigma_{2}, \sigma_{3}, \sigma_{4}, \sigma_{5}, \sigma_{6}\right\}^{T}, \\
\{\epsilon\}=\left\{\epsilon_{1}, \epsilon_{2}, \epsilon_{3}, \epsilon_{4}, \epsilon_{5}, \epsilon_{6}\right\}^{T} .
\end{gathered}
$$

La ecuación constitutiva es:

$$
\{\sigma\}=[C]\{\epsilon\}
$$

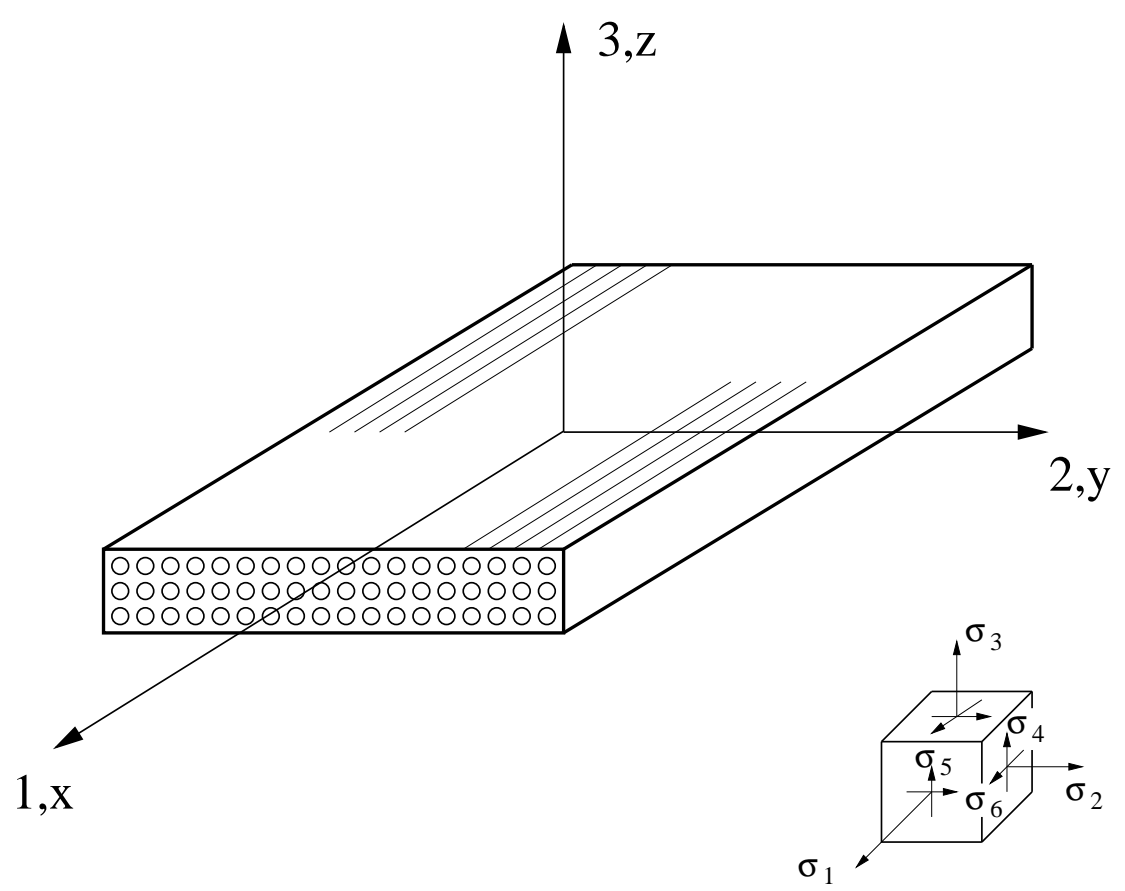

Figura 2.1: Tensor de tensiones en una placa unidireccional cuando las coordenadas globales y locales coinciden con las direcciones ortótropas

La matriz de rigidez $[C]$ tiene dimensión $(6 \times 6)$ así que en su expresión más general tiene 36 componentes. Sin embargo sólo 21 términos son independientes ya que esta matriz es simétrica como se demuestra a continuación.

Para los materiales elásticos la energía de deformación por unidad de volumen vale

$$
d W=\sigma_{i} d \epsilon_{i}
$$

Sustituyendo (2.5) en (2.6), de las relaciones entre tensión y deformación pasamos a

$$
d W=C_{i j} \epsilon_{j} d \epsilon_{i}
$$

que integrada obtenemos

$$
W=\frac{1}{2} C_{i j} \epsilon_{i} \epsilon_{j} .
$$


Derivando dos veces llegamos a

$$
\frac{\partial^{2} W}{\partial \epsilon_{i} \partial \epsilon_{j}}=C_{i j}
$$

De forma análoga podemos llegar a

$$
\frac{\partial^{2} W}{\partial \epsilon_{j} \partial \epsilon_{i}}=C_{j i}
$$

pero como el orden de derivación de $W$ es indiferente, se obtiene que

$$
C_{i j}=C_{j i}
$$

Por tanto, para los materiales anisótropos la relación tensión-deformación queda como

$$
\left\{\begin{array}{c}
\sigma_{1} \\
\sigma_{2} \\
\sigma_{3} \\
\tau_{23} \\
\tau_{13} \\
\tau_{12}
\end{array}\right\}=\left[\begin{array}{cccccc}
C_{11} & C_{12} & C_{13} & C_{14} & C_{15} & C_{16} \\
& C_{22} & C_{23} & C_{24} & C_{25} & C_{26} \\
& & C_{33} & C_{34} & C_{35} & C_{36} \\
& & & C_{44} & C_{45} & C_{46} \\
& & & & C_{55} & C_{56} \\
& & & & & C_{66}
\end{array}\right]\left\{\begin{array}{c}
\epsilon_{1} \\
\epsilon_{2} \\
\epsilon_{3} \\
\gamma_{23} \\
\gamma_{13} \\
\gamma_{12}
\end{array}\right\}
$$

En presencia de un plano de simetría para las propiedades del material, la relación tensión deformación queda

$$
\left\{\begin{array}{c}
\sigma_{1} \\
\sigma_{2} \\
\sigma_{3} \\
\tau_{23} \\
\tau_{13} \\
\tau_{12}
\end{array}\right\}=\left[\begin{array}{cccccc}
C_{11} & C_{12} & C_{13} & 0 & 0 & C_{16} \\
& C_{22} & C_{23} & 0 & 0 & C_{26} \\
& & C_{33} & 0 & 0 & C_{36} \\
& & & C_{44} & C_{45} & 0 \\
& & & & C_{55} & 0 \\
& & & & & C_{66}
\end{array}\right]\left\{\begin{array}{c}
\epsilon_{1} \\
\epsilon_{2} \\
\epsilon_{3} \\
\gamma_{23} \\
\gamma_{13} \\
\gamma_{12}
\end{array}\right\}
$$

donde el plano de simetría es el $z=0$. Para este tipo de materiales hay 13 constantes elásticas independientes. Con dos planos de simetría ortogonales al material se le designa ortótropo y la relación queda 


$$
\left\{\begin{array}{c}
\sigma_{1} \\
\sigma_{2} \\
\sigma_{3} \\
\tau_{23} \\
\tau_{13} \\
\tau_{12}
\end{array}\right\}=\left[\begin{array}{cccccc}
C_{11} & C_{12} & C_{13} & 0 & 0 & 0 \\
& C_{22} & C_{23} & 0 & 0 & 0 \\
& & C_{33} & 0 & 0 & 0 \\
& & & C_{44} & 0 & 0 \\
& & & & C_{55} & 0 \\
& & & & & C_{66}
\end{array}\right]\left\{\begin{array}{c}
\epsilon_{1} \\
\epsilon_{2} \\
\epsilon_{3} \\
\gamma_{23} \\
\gamma_{13} \\
\gamma_{12}
\end{array}\right\}
$$

Los materiales compuestos unidireccionales presentan dos planos de simetría ortogonales, uno de ellos en la dirección de las fibras y el otro perpendicular a ellas. Cuando las tensiones y deformaciones se miden en la dirección de las fibras, se considera al material ortótropo y $[C]$ sólo tiene 9 términos independientes. Las propiedades del material se miden habitualmente mediante la matriz de flexibilidad $[S]=[C]^{-1}$. Debido al tipo de ensayos que caracterizan al material las componentes de $[S]$ se expresan en términos de constantes de ingeniería de la forma

$$
\left\{\begin{array}{c}
\epsilon_{1} \\
\epsilon_{2} \\
\epsilon_{3} \\
\gamma_{23} \\
\gamma_{13} \\
\gamma_{12}
\end{array}\right\}=\left[\begin{array}{cccccc}
\frac{1}{E_{1}} & -\frac{\nu_{12}}{E_{2}} & -\frac{\nu_{13}}{E_{3}} & 0 & 0 & 0 \\
& \frac{1}{E_{2}} & -\frac{\nu_{23}}{E_{3}} & 0 & 0 & 0 \\
& & \frac{1}{E_{3}} & 0 & 0 & 0 \\
& & & \frac{1}{G_{23}} & 0 & 0 \\
& & & & \frac{1}{G_{13}} & 0 \\
& & & & & \frac{1}{G_{12}}
\end{array}\right]\left\{\begin{array}{c}
\sigma_{1} \\
\sigma_{2} \\
\sigma_{3} \\
\tau_{23} \\
\tau_{13} \\
\tau_{12}
\end{array}\right\} .
$$

Las propiedades del material en las direcciones 2 y 3 dependen ambas principalmente de la matriz (fase aglomerante) del material compuesto por lo que

$$
E_{3}=E_{2} ; \quad G_{13}=G_{12} .
$$

Además, por las condiciones de simetría sabemos que

$$
\frac{\nu_{i j}}{E_{i}}=\frac{\nu_{j i}}{E_{j}} ; \quad i, j=1,2,3
$$

con lo que aplicando las dos ecuaciones anteriores tenemos

$$
\nu_{13}=\nu_{12}
$$


También sabemos que al ser el material isótropo en la dirección perpendicular a la fibra, se cumple que

$$
S_{44}=2\left(S_{22}-S_{23}\right) \quad \Rightarrow \quad G_{23}=\frac{E_{2}}{2\left(1+\nu_{23}\right)} .
$$

Esta última relación procede de la equivalencia de estados de tensión de cortante puro y de tracción-compresión puro y sólo es valida para la dirección en la que las propiedades son isótropas.

Por tanto, y resumiendo, podemos decir que el número de constantes elásticas del material en tres dimensiones se reducen a cinco:

$$
E_{1}, E_{2}, \nu_{12}, \nu_{23}, G_{12}
$$

\section{Propiedades del material en ejes rotados}

Cuando el sistema de referencia global $(1-2-3)$ no está orientado en la misma dirección (de las fibras) que el sistema de referencia local (x-y-z), Figura 2.2, el material ya no es ortótropo y hay acoplamiento entre los esfuerzos y deformaciones normales y angulares. Estudiamos dos tipos de rotaciones, por un lado tendremos un tensor de segundo orden para rotar las tensiones, deformaciones y fuerzas y por otro un tensor de cuarto orden para la rotación de las matrices de rigidez.

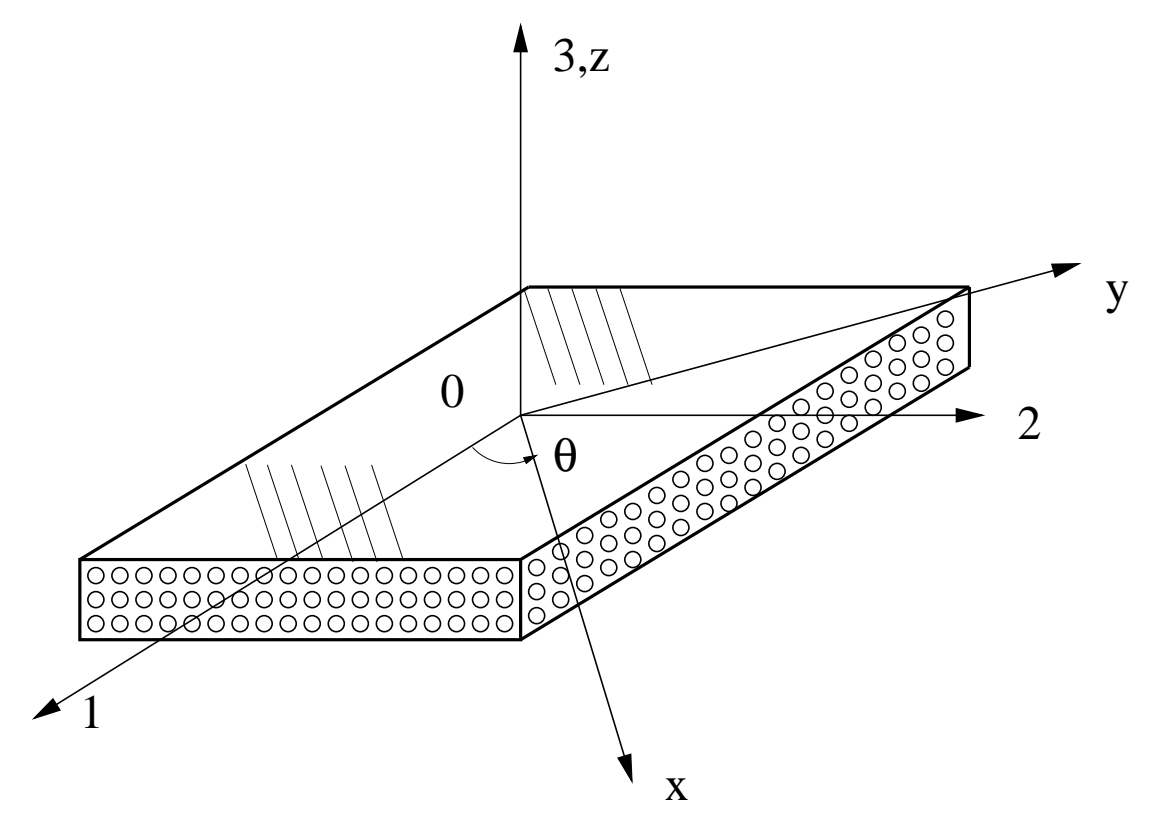

Figura 2.2: Rotación de los ejes de coordenadas desde un sistema de referencia global $1-2-3$ hasta un sistema local $x-y-z$

La transformación de tensiones correspondiente a una rotación de ángulo $\theta$ alrededor 
del punto $O$, desde coordenadas globales a locales es:

$$
\begin{gathered}
{[\sigma]_{x-y-z}=[\beta][\sigma]_{1-2-3}[\beta]^{T},} \\
{[\beta]=\left[\begin{array}{ccc}
m & n & 0 \\
-n & m & 0 \\
0 & 0 & 1
\end{array}\right] \quad\left\{\begin{array}{l}
m=\cos \theta \\
n=\operatorname{sen} \theta
\end{array} .\right.}
\end{gathered}
$$

Esta relación se puede expresar de forma matricial como

$$
\left\{\begin{array}{c}
\sigma_{1} \\
\sigma_{2} \\
\sigma_{3} \\
\tau_{23} \\
\tau_{13} \\
\tau_{12}
\end{array}\right\}_{x-y-z}=\left[\begin{array}{cccccc}
m^{2} & n^{2} & 0 & 0 & 0 & 2 m n \\
n^{2} & m^{2} & 0 & 0 & 0 & -2 m n \\
0 & 0 & 1 & 0 & 0 & 0 \\
0 & 0 & 0 & m & -n & 0 \\
0 & 0 & 0 & n & m & 0 \\
-m n & m n & 0 & 0 & 0 & m^{2}-n^{2}
\end{array}\right]\left\{\begin{array}{c}
\sigma_{1} \\
\sigma_{2} \\
\sigma_{3} \\
\tau_{23} \\
\tau_{13} \\
\tau_{12}
\end{array}\right\}_{1-2-3}
$$

La rotación contraria se puede calcular mediante la inversa de esta matriz o mediante un giro de valor $-\theta$ en vez de $\theta$, obteniendo las siguientes expresiones

$$
\begin{gathered}
\left\{\begin{array}{c}
\sigma_{1} \\
\sigma_{2} \\
\sigma_{3} \\
\tau_{23} \\
\tau_{13} \\
\tau_{12}
\end{array}\right\}_{1-2-3}=\left[\begin{array}{cccccc}
m^{2} & n^{2} & 0 & 0 & 0 & -2 m n \\
n^{2} & m^{2} & 0 & 0 & 0 & 2 m n \\
0 & 0 & 1 & 0 & 0 & 0 \\
0 & 0 & 0 & m & n & 0 \\
0 & 0 & 0 & -n & m & 0 \\
m n & -m n & 0 & 0 & 0 & m^{2}-n^{2}
\end{array}\right]\left\{\begin{array}{c}
\sigma_{1} \\
\sigma_{2} \\
\sigma_{3} \\
\tau_{23} \\
\tau_{13} \\
\tau_{12}
\end{array}\right\}_{x-y-z}, \\
\{\sigma\}_{1-2-3}=[T]^{-1}\{\sigma\}_{x-y-z}
\end{gathered}
$$

Para hallar las transformaciones de las deformaciones debemos tener en cuenta la diferencia entre deformaciones tensoriales e ingenieriles. La relación entre ambas es

$$
\begin{aligned}
& \{\epsilon\}_{x-y-z}=[R]\{e\}_{x-y-z}, \\
& \{\epsilon\}_{1-2-3}=[R]\{e\}_{1-2-3},
\end{aligned}
$$

donde $\{e\}$ es la deformación tensorial y $\{\epsilon\}$ la deformación ingenieril, ambas expresadas en coordenadas globales o locales. $[R]$ es la matriz de Reuter que consiste en una matriz diagonal con 1 en las filas de las deformaciones lineales y 2 en las filas de las deformaciones 
angulares. La relación entre deformaciones es

$$
\begin{gathered}
\left\{\begin{array}{c}
\epsilon_{1} \\
\epsilon_{2} \\
\epsilon_{3} \\
\gamma_{23} \\
\gamma_{13} \\
\gamma_{12}
\end{array}\right\}_{x-y-z}=\left[\begin{array}{cccccc}
m^{2} & n^{2} & 0 & 0 & 0 & m n \\
n^{2} & m^{2} & 0 & 0 & 0 & -m n \\
0 & 0 & 1 & 0 & 0 & 0 \\
0 & 0 & 0 & m & -n & 0 \\
0 & 0 & 0 & n & m & 0 \\
-2 m n & 2 m n & 0 & 0 & 0 & m^{2}-n^{2}
\end{array}\right]\left\{\begin{array}{c}
\epsilon_{1} \\
\epsilon_{2} \\
\epsilon_{3} \\
\gamma_{23} \\
\gamma_{13} \\
\gamma_{12}
\end{array}\right\}_{1-2-3}, \\
\{\epsilon\}_{x-y-z}=[R][T]^{-1}\{\epsilon]_{1-2-3}, \\
\{\epsilon\}_{x-y-z}=[T]^{-T}\{\epsilon\}_{1-2-3} .
\end{gathered}
$$

Igual que en el caso de las tensiones se puede obtener fácilmente la transformación inversa

$$
\begin{aligned}
& \left\{\begin{array}{c}
\epsilon_{1} \\
\epsilon_{2} \\
\epsilon_{3} \\
\gamma_{23} \\
\gamma_{13} \\
\gamma_{12}
\end{array}\right\}_{1-2-3}=\left[\begin{array}{cccccc}
m^{2} & n^{2} & 0 & 0 & 0 & -m n \\
n^{2} & m^{2} & 0 & 0 & 0 & m n \\
0 & 0 & 1 & 0 & 0 & 0 \\
0 & 0 & 0 & m & n & 0 \\
0 & 0 & 0 & -n & m & 0 \\
2 m n & -2 m n & 0 & 0 & 0 & m^{2}-n^{2}
\end{array}\right]\left\{\begin{array}{c}
\epsilon_{1} \\
\epsilon_{2} \\
\epsilon_{3} \\
\gamma_{23} \\
\gamma_{13} \\
\gamma_{12}
\end{array}\right\}_{x-y-z} \\
& \{\epsilon\}_{1-2-3}=[T]^{T}\{\epsilon\}_{x-y-z} .
\end{aligned}
$$

La última transformación que veremos es la de la matriz de rigidez que será la que finalmente implementaremos en el programa de elementos finitos. Como ya vimos la ecuación constitutiva en coordenadas locales es

$$
\left\{\begin{array}{c}
\sigma_{1} \\
\sigma_{2} \\
\sigma_{3} \\
\tau_{23} \\
\tau_{13} \\
\tau_{12}
\end{array}\right\}_{x-y-z}=\left[\begin{array}{cccccc}
C_{11} & C_{12} & C_{12} & 0 & 0 & 0 \\
C_{12} & C_{22} & C_{23} & 0 & 0 & 0 \\
C_{12} & C_{23} & C_{22} & 0 & 0 & 0 \\
0 & 0 & 0 & C_{44} & 0 & 0 \\
0 & 0 & 0 & 0 & C_{55} & 0 \\
0 & 0 & 0 & 0 & 0 & C_{55}
\end{array}\right]_{x-y-z} \quad\left\{\begin{array}{c}
\epsilon_{1} \\
\epsilon_{2} \\
\epsilon_{3} \\
\gamma_{23} \\
\gamma_{13} \\
\gamma_{12}
\end{array}\right\}_{x-y-z}
$$


Aplicando a la relación constitutiva (2.31) las transformaciones de giro tensorial (2.23) y (2.28) se obtiene

$$
\begin{aligned}
\{\sigma\}_{x-y-z} & =[C]_{x-y-z}\{\epsilon\}_{x-y-z} \\
{[T]\{\sigma\}_{1-2-3} } & =[C]_{x-y-z}[T]^{-T}\{\epsilon\}_{1-2-3}, \\
\{\sigma\}_{1-2-3} & =[T]^{-1}[C]_{x-y-z}[T]^{-T}\{\epsilon\}_{1-2-3} \\
\{\sigma\}_{1-2-3} & =[C]_{1-2-3}\{\epsilon\}_{1-2-3} \\
{[C]_{1-2-3} } & =[T]^{-1}[C]_{x-y-z}[T]^{-T}
\end{aligned}
$$

y en forma matricial:

$$
\left\{\begin{array}{c}
\sigma_{1} \\
\sigma_{2} \\
\sigma_{3} \\
\tau_{23} \\
\tau_{13} \\
\tau_{12}
\end{array}\right\}_{1-2-3}=\left[\begin{array}{cccccc}
C_{11} & C_{12} & C_{13} & 0 & 0 & C_{16} \\
C_{12} & C_{22} & C_{23} & 0 & 0 & C_{26} \\
C_{13} & C_{23} & C_{33} & 0 & 0 & C_{36} \\
0 & 0 & 0 & C_{44} & C_{45} & 0 \\
0 & 0 & 0 & C_{45} & C_{55} & 0 \\
C_{16} & C_{26} & C_{36} & 0 & 0 & C_{66}
\end{array}\right]_{1-2-3} \quad\left\{\begin{array}{c}
\epsilon_{1} \\
\epsilon_{2} \\
\epsilon_{3} \\
\gamma_{23} \\
\gamma_{13} \\
\gamma_{12}
\end{array}\right\}_{1-2-3}
$$

Desarrollando las ecuaciones (2.32) y (2.33) se obtiene que:

$$
\left\{\begin{array}{l}
C_{11} \\
C_{22} \\
C_{12} \\
C_{33} \\
C_{13} \\
C_{23} \\
C_{16} \\
C_{26} \\
C_{36} \\
C_{44} \\
C_{55} \\
C_{45} \\
C_{66}
\end{array}\right\}=\left[\begin{array}{cccccc}
m^{4} & n^{4} & 2 m^{2} n^{2} & 0 & 0 & 4 m^{2} n^{2} \\
n^{4} & m^{4} & 2 m^{2} n^{2} & 0 & 0 & 4 m^{2} n^{2} \\
m^{2} n^{2} & m^{2} n^{2} & m^{4}+n^{4} & 0 & 0 & -4 m^{2} n^{2} \\
0 & 1 & 0 & 0 & 0 & 0 \\
0 & 0 & m^{2} & n^{2} & 0 & 0 \\
0 & 0 & n^{2} & m^{2} & 0 & 0 \\
m^{3} n & -m n^{3} & m n^{3}-m^{3} n & 0 & 0 & 2\left(m n^{3}-m^{3} n\right) \\
m n^{3} & -m^{3} n & m^{3} n-m n^{3} & 0 & 0 & 2\left(m^{3} n-m n^{3}\right) \\
0 & 0 & m n & -m n & 0 & 0 \\
0 & 0 & 0 & 0 & m^{2} & n^{2} \\
0 & 0 & 0 & 0 & n^{2} & m^{2} \\
m^{2} n^{2} & m^{2} n^{2} & -2 m^{2} n^{2} & 0 & 0 & \left(m^{2}-n^{2}\right)^{2}
\end{array}\right]\left\{\begin{array}{c}
C_{11} \\
C_{22} \\
C_{12} \\
C_{23} \\
C_{44} \\
C_{55}
\end{array}\right\}
$$

donde el primer vector contiene rigideces en el sistema de coordenadas $\{1-2-3\}$ y el segundo en el $\{x-y-z\}$. 


\subsubsection{Descripción bidimensional}

En esta sección las ecuaciones constitutivas anteriores se describen en dos dimensiones [8]. La rigidez de los materiales compuestos unidireccionales, como la del resto de los materiales estructurales, puede definirse mediante relaciones tensión-deformación. Para desarrollar estas relaciones debemos saber que cuando hablamos de tensión en los materiales compuestos debemos definirla a tres niveles. Por un lado, a escala microscópica tenemos una definición que proviene de distinguir la tensión en las fibras, en la matriz, y en algunos caso en la unión entre capas. Por otro, la media de estas tensiones es la tensión macroscópica de capa. Y por último tenemos el esfuerzo unitario normal resultante $N$ y el momento resultante $M$ como media en un laminado de las tensiones de capa.

El estado de tensión habitual en un laminado de material compuesto es el estado plano de tensiones, ya que se construyen en forma de placa. Para los materiales compuestos debemos respetar cuidadosamente el convenio de signos en las tensiones (véase la Figura 2.3) pues la diferencia entre las resistencias a tracción y compresión pueden ser grande.

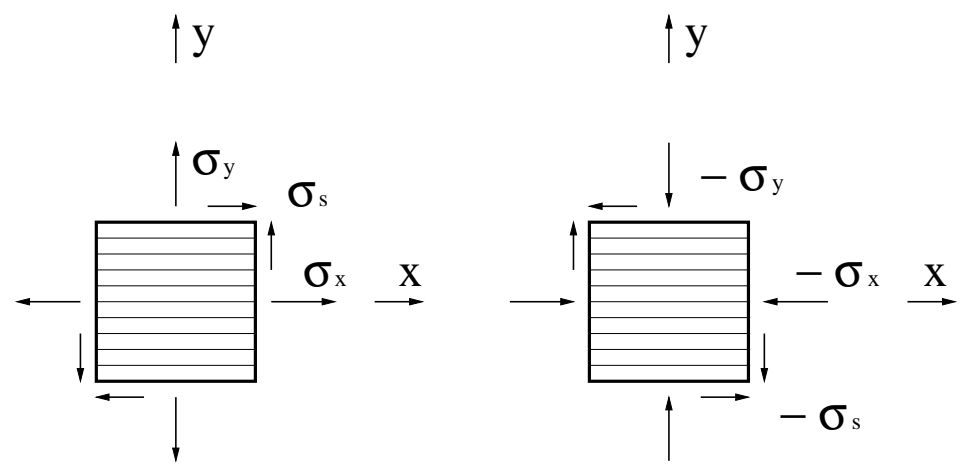

Figura 2.3: Criterio de signos

Las deformaciones las definimos como la variación espacial de los desplazamientos. Siendo $u$ un desplazamiento a lo largo de la dirección $x$ y $v$ un desplazamiento a lo largo de la dirección $y$, podemos definir:

$$
\begin{gathered}
\epsilon_{x}=\lim _{\Delta x \rightarrow 0} \frac{\Delta u}{\Delta x}=\frac{\partial u}{\partial x} \\
\epsilon_{y}=\lim _{\Delta y \rightarrow 0} \frac{\Delta v}{\Delta y}=\frac{\partial v}{\partial y} \\
\epsilon_{s}=\frac{\partial v}{\partial x}+\frac{\partial v}{\partial y} .
\end{gathered}
$$

en donde $\epsilon_{s}$ es la deformación angular ingenieril, dos veces la tensorial, utilizada porque mide la deformación angular total.

Como dijimos el estudio de este trabajo se basa en el comportamiento elástico lineal de 
los materiales compuestos. La linealidad nos permite utilizar el principio de superposición y la elasticidad implica la ausencia de ciclos de histéresis o deformaciones permanentes. Experimentalmente se comprueba que el comportamiento de los materiales compuestos se ajusta más a la elasticidad lineal que casi todos los metales y los plásticos. Como se comentó en el apartado anterior, para los compuestos unidireccionales existen dos planos de simetría ortogonales: uno paralelo a las fibras y otro transversal a ellas. Cuando los ejes de referencia coinciden con los ejes de simetría del material es relativamente sencillo construir la matriz de elasticidad del material

$$
\left\{\begin{array}{c}
\epsilon_{x} \\
\epsilon_{y} \\
\epsilon_{s}
\end{array}\right\}=\left[\begin{array}{ccc}
\frac{1}{E_{x}} & -\frac{\nu_{y}}{E_{y}} & 0 \\
-\frac{\nu_{x}}{E_{x}} & \frac{1}{E_{y}} & 0 \\
0 & 0 & \frac{1}{E_{s}}
\end{array}\right]\left\{\begin{array}{c}
\sigma_{x} \\
\sigma_{y} \\
\sigma_{s}
\end{array}\right\}=\left[\begin{array}{ccc}
S_{x x} & S_{x y} & 0 \\
S_{y x} & S_{y y} & 0 \\
0 & 0 & S_{s s}
\end{array}\right]\left\{\begin{array}{c}
\sigma_{x} \\
\sigma_{y} \\
\sigma_{s}
\end{array}\right\} .
$$

Si invertimos la matriz de flexibilidades obtenemos la matriz de rigidez

$$
\left\{\begin{array}{c}
\sigma_{x} \\
\sigma_{y} \\
\sigma_{s}
\end{array}\right\}=\left[\begin{array}{ccc}
Q_{x x} & Q_{x y} & 0 \\
Q_{y x} & Q_{y y} & 0 \\
0 & 0 & Q_{s s}
\end{array}\right]\left\{\begin{array}{l}
\epsilon_{x} \\
\epsilon_{y} \\
\epsilon_{s}
\end{array}\right\}
$$

donde si definimos $p=\left[1-\nu_{x} \nu_{y}\right]^{-1}$ podemos llegar a las expresiones

$$
\left\{\begin{array}{cl}
Q_{x x}=p E_{x} ; & Q_{x x}=p E_{x} ; \quad Q_{s s}=E_{s} \\
Q_{y x}=p \nu_{x} E_{y} ; & Q_{x y}=p \nu_{y} E_{x}
\end{array}\right.
$$

En el caso de ejes rotados la matriz $[T]$ será

$$
[T]=\left[\begin{array}{ccc}
m^{2} & n^{2} & 2 m n \\
n^{2} & m^{2} & -2 m n \\
-m n & m n & m^{2}-n^{2}
\end{array}\right]
$$

y todos las ecuaciones matriciales serán análogas al caso tridimensional, de forma que las relaciones entre tensión y deformación serán:

$$
\begin{gathered}
\{\sigma\}_{1-2-3}=[T]^{-1}[C]_{x-y-z}[T]^{-T}\{\epsilon\}_{1-2-3} \\
\{\epsilon\}_{1-2-3}=[T]^{T}[S]_{x-y-z}[T]\{\sigma\}_{1-2-3} .
\end{gathered}
$$

La matriz $[S]$ nos indica que en caso de una placa unidireccional con las fibras orienta- 
das en la dirección de los ejes principales, al aplicar una fuerza de tracción/compresión en la dirección de las fibras o en la dirección perpendicular a las fibras $\left(\sigma_{x}\right.$ y $\sigma_{y}$ distintas de cero) la placa sufrirá un cambio en sus dimensiones (acortamiento o estrechamiento), pero no sufrirá deformación angular, y si aplicamos una fuerza de tipo cortante $\left(\sigma_{s}\right.$ distinta de cero) la placa sufrirá deformación angular pero no cambios en sus dimensiones.

Sin embargo, si la dirección de las fibras en la placa unidireccional es diferente de cero o noventa grados, la placa sufrirá además de un cambio en sus dimensiones una deformación angular, y si aplicamos un cortante sufrirá un cambio en sus dimensiones además de una deformación angular.

\subsection{Cálculo de las frecuencias propias}

En esta sección se presenta el procedimiento analítico para el cálculo de las frecuencias propias de vibración para placas unidireccionales. El cálculo es válido para cualquier ángulo de orientación de las fibras. Para placas multidireccionales no es posible calcular las frecuencias propias analíticamente.

Para calcular las frecuencias propias se ha usado el método de Ritz, basado en consideraciones energéticas. Un cálculo más detallado se puede ver en [11].

Este método nos dice que en vibraciones libres, en ausencia de cargas laterales y en el propio plano de la placa, podemos suponer que

$$
U-T=\text { valor estacionario, }
$$

siendo $U$ la energía de deformación y $T$ la energía cinética total de la placa.

En estas condiciones

$$
\begin{gathered}
U=\frac{1}{2} \int_{0}^{b} \int_{0}^{a}\left[D_{11}\left(\frac{\partial^{2} \mathrm{w}}{\partial x^{2}}\right)^{2}+2 D_{12} \frac{\partial^{2} \mathrm{w}}{\partial x^{2}} \frac{\partial^{2} \mathrm{w}}{\partial y^{2}}+D_{22}\left(\frac{\partial^{2} \mathrm{w}}{\partial y^{2}}\right)^{2}+\right. \\
\left.+4 D_{66}\left(\frac{\partial^{2} \mathrm{w}}{\partial x \partial y}\right)^{2}+4 D_{16} \frac{\partial^{2} \mathrm{w}}{\partial x^{2}} \frac{\partial^{2} \mathrm{w}}{\partial x \partial y}+4 D_{26} \frac{\partial^{2} \mathrm{w}}{\partial y^{2}} \frac{\partial^{2} \mathrm{w}}{\partial x \partial y}\right] \mathrm{d} x \mathrm{~d} y \\
T=\frac{1}{2} \int_{0}^{b} \int_{0}^{a} \rho \omega^{2} \mathrm{w}^{2} \mathrm{~d} x \mathrm{~d} y
\end{gathered}
$$

donde

$$
D_{i j}=\int_{-h / 2}^{h / 2} Q_{i j} z^{2} \mathrm{~d} z,
$$

$a, b$ y $h$ las dimensiones de la placa, $\rho$ la densidad por unidad de superficie, w(x,y) el desplazamiento en el eje z y $\omega=2 \pi f$, siendo $f$ las frecuencias que buscamos. 
Suponemos una solución para w del tipo

$$
\mathrm{w}(x, y)=\sum_{m=1}^{M} \sum_{n=1}^{N} A_{m n} X_{m}(x) Y_{n}(y),
$$

siendo $X_{m}(x)$ y $Y_{n}(y)$ funciones que satisfacen las condiciones de contorno en los cuatro lados de la placa.

Usando (2.48) en (2.44), podemos decir que

$$
\frac{\partial(U-T)}{\partial A_{m n}}=0 ; \quad m=1, \ldots, M ; \quad n=1, \ldots, N
$$

Si combinamos (2.48), (2.45), (2.46) y (2.49) obtenemos

$$
\begin{gathered}
\sum_{i=1}^{M} \sum_{j=1}^{N}\left\{D_{11} \int_{0}^{a} \frac{\mathrm{d}^{2} X_{i}}{\mathrm{~d} x^{2}} \frac{\mathrm{d}^{2} X_{m}}{\mathrm{~d} x^{2}} \mathrm{~d} x \int_{0}^{b} Y_{j} Y_{n} \mathrm{~d} y\right. \\
+D_{12}\left[\int_{0}^{a} \frac{\mathrm{d}^{2} X_{i}}{\mathrm{~d} x^{2}} X_{m} \mathrm{~d} x \int_{0}^{b} Y_{j} \frac{\mathrm{d}^{2} Y_{n}}{\mathrm{~d} y^{2}} \mathrm{~d} y+\int_{0}^{a} X_{i} \frac{\mathrm{d}^{2} X_{m}}{\mathrm{~d} x^{2}} \mathrm{~d} x \int_{0}^{b} \frac{\mathrm{d}^{2} Y_{j}}{\mathrm{~d} y^{2}} Y_{n} \mathrm{~d} y\right] \\
+D_{22} \int_{0}^{a} X_{i} X_{m} \mathrm{~d} x \int_{0}^{b} \frac{\mathrm{d}^{2} Y_{j}}{\mathrm{~d} y^{2}} \frac{\mathrm{d}^{2} Y_{n}}{\mathrm{~d} y^{2}} \mathrm{~d} y \\
+4 D_{66} \int_{0}^{a} \frac{\mathrm{d} X_{i}}{\mathrm{~d} x} \frac{\mathrm{d} X_{m}}{\mathrm{~d} x} \mathrm{~d} x \int_{0}^{b} \frac{\mathrm{d} Y_{j}}{\mathrm{~d} y} \frac{\mathrm{d} Y_{n}}{\mathrm{~d} y} \mathrm{~d} y \\
+2 D_{16}\left[\int_{0}^{a} \frac{\mathrm{d}^{2} X_{i}}{\mathrm{~d} x^{2}} \frac{\mathrm{d} X_{m}}{\mathrm{~d} x} \mathrm{~d} x \int_{0}^{b} Y_{j} \frac{\mathrm{d} Y_{n}}{\mathrm{~d} y} \mathrm{~d} y+\int_{0}^{a} \frac{\mathrm{d} X_{i}}{\mathrm{~d} x} \frac{\mathrm{d}^{2} X_{m}}{\mathrm{~d} x^{2}} \mathrm{~d} x \int_{0}^{b} \frac{\mathrm{d} Y_{j}}{\mathrm{~d} y} Y_{n} \mathrm{~d} y\right] \\
{\left[\int_{0}^{a} \frac{\mathrm{d} X_{i}}{\mathrm{~d} x} X_{m} \mathrm{~d} x \int_{0}^{b} \frac{\mathrm{d} Y_{j}}{\mathrm{~d} y} \frac{\mathrm{d}^{2} Y_{n}}{\mathrm{~d} y^{2}} \mathrm{~d} y+\int_{0}^{a} X_{i} \frac{\mathrm{d} X_{m}}{\mathrm{~d} x} \mathrm{~d} x \int_{0}^{b} \frac{\mathrm{d}^{2} Y_{j}}{\mathrm{~d} y^{2}} \frac{\mathrm{d} Y_{n}}{\mathrm{~d} y} \mathrm{~d} y\right]} \\
\left.-\rho \omega^{2} \int_{0}^{a} X_{i} X_{m} \mathrm{~d} x \int_{0}^{b} Y_{j} Y_{n} \mathrm{~d} y\right\} A_{i j}=0 ; \\
m=1, \ldots, M ; n=1, \ldots, N .
\end{gathered}
$$

Nos encontramos ante un sistema de ecuaciones homogéneo, cuya solución sólamente será distinta de la trivial si el determinante de la matriz de coeficientes es cero. Las soluciones que obtengamos al igualar este determinante a cero, serán las que nos den las frecuencias propias de vibración de la placa, una vez determinadas las funciones $X_{m}(x)$ e $Y_{n}(y)$.

Si la placa está simplemente apoyada en sus cuatro lados podemos tomar

$$
\begin{aligned}
& X_{m}(x)=\sin \frac{m \pi x}{a}, \\
& Y_{n}(y)=\sin \frac{n \pi y}{b} .
\end{aligned}
$$


Si la placa está empotrada en sus cuatro lados podemos tomar

$$
\begin{gathered}
X_{m}(x)=\cosh \frac{\lambda_{m} x}{a}-\cos \frac{\lambda_{m} x}{a}-\gamma_{m}\left(\sinh \frac{\lambda_{m} x}{a}-\sin \frac{\lambda_{m} x}{a}\right), \\
Y_{n}(y)=\cosh \frac{\lambda_{n} y}{a}-\cos \frac{\lambda_{n} y}{a}-\gamma_{n}\left(\sinh \frac{\lambda_{n} y}{a}-\sin \frac{\lambda_{n} y}{a}\right),
\end{gathered}
$$

donde $\lambda_{m}$ y $\lambda_{n}$ son las soluciones de la ecuación

$$
\cos \left(\lambda_{i}\right) \cosh \left(\lambda_{i}\right)=1
$$

$\mathrm{y}$

$$
\gamma_{i}=\frac{\cos \left(\lambda_{i}\right)-\cosh \left(\lambda_{i}\right)}{\sin \left(\lambda_{i}\right)+\sinh \left(\lambda_{i}\right)}
$$

Si la placa tiene dos lados contiguos simplemente apoyados y los otros dos lados empotrados, podemos tomar $X_{m}(x)$ e $Y_{n}(y)$ de las ecuaciones $(2.53)$ y $(2.54)$, pero ahora $\lambda_{m}$ y $\lambda_{n}$ son las soluciones de la ecuación

$$
\tan \left(\lambda_{i}\right)-\tanh \left(\lambda_{i}\right)=0
$$

y

$$
\gamma_{i}=\frac{\sin \left(\lambda_{i}\right)-\sinh \left(\lambda_{i}\right)}{\cosh \left(\lambda_{i}\right)-\cos \left(\lambda_{i}\right)}
$$

Introduciendo $X_{m}(x)$ e $Y_{n}(y)$ según las condiciones de contorno en el sistema de ecuaciones (2.50), y considerando lo anteriormente dicho para que este sistema tenga solución, podemos obtener las frecuencias propias de la placa.

\subsection{Notación para placas multidireccionales}

Las secuencias de apilado en placas se pueden describir fácilmente, para capas del mismo material y espesor, y distintas orientaciones de las fibras, como es el caso habitual. Cada capa vendrá representada por un número, que indica el ángulo (en grados) que forman las fibras respecto a los ejes naturales de la placa. Así, por ejemplo [0/90/0] define una placa de tres capas, a cero, noventa y cero grados respectivamente. Si alguna capa estuviera repetida, se indica mediante un subíndice. Por ejemplo $\left[0 / 90_{3} / 0\right]$ indica que la capa con las fibras a 90 grados está repetida tres veces. Para placas simétricas, como por ejemplo $\left[0 / 90_{2} / 0\right]$, podemos abreviar la notación usando $[0 / 90]_{s}$, donde el subíndice $s$ denota que la secuencia se repite simétricamente. Otras configuraciones como [0/45/-45] pueden ser abreviadas como $[0 / \pm 45]$. Si lo que se repite es un conjunto de placas, como 
$[0 / \pm 45 / 0 / \pm 45]_{s}$, podemos abreviar como $[0 / \pm 45]_{2 s}$.

Por ejemplo, la disposición $\left[0_{3} / 90_{2} / 45 /-45_{3}\right]_{s}$ se esquematiza en la Figura 2.4.

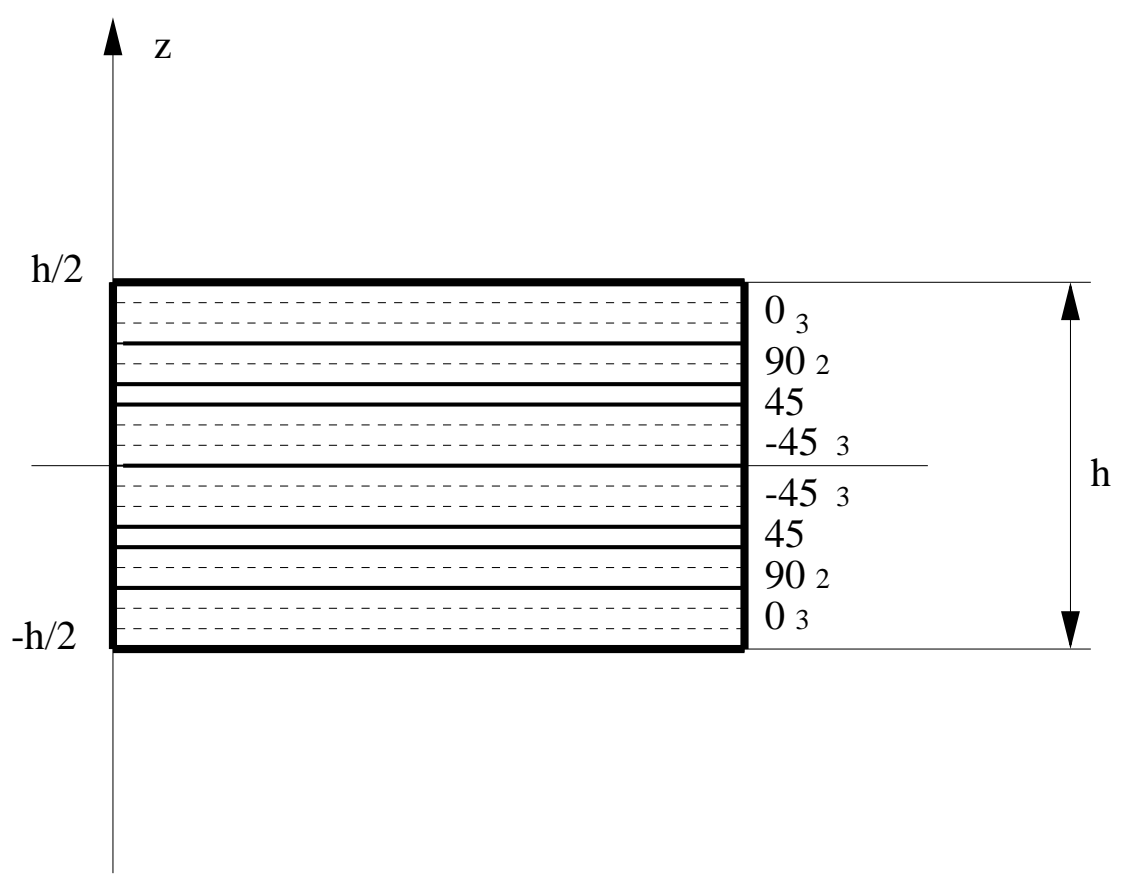

Figura 2.4: Ejemplo de apilado definido según el código $\left[0_{3} / 90_{2} / 45 /-45_{3}\right]_{s}$ 


\section{Capítulo 3}

\section{El método de los elementos finitos}

Como se ha comentado en la introducción, en este trabajo se va a realizar un estudio numérico de placas de material compuesto mediante el Método de los Elementos Finitos (MEF). Por ello, en el presente Capítulo se hace un repaso histórico del MEF y se formula el método de manera general. En la última parte del mismo se explican los diferentes programas de elementos finitos utilizados.

\subsection{Historia del MEF}

El nacimiento del MEF proviene de contribuciones independientes en los campos de la

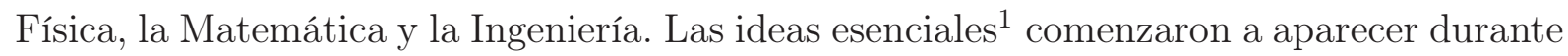
la década de los 40:

- Matemática: En 1943 Courant definió ecuaciones sobre una región triangularizada para resolver un problema de torsión en Elasticidad. En 1946 Schoenberg da a luz la teoría de los splines, recomendando su uso como polinomios para aproximación e interpolación. En 1959 Greenstadt dividió un dominio en celdas, asignando una función diferente a cada una y aplicando principios variacionales. White y Friedrichs utilizaron elementos triangulares para resolver ecuaciones diferenciales mediante principios variacionales también.

- Física: Prager y Synge interpretaron geométricamente los principios del mínimo de la Teoría clásica de Elasticidad. Synge usó funciones lineales definidas sobre una región triangularizada con el procedimiento variacional de Ritz. Por otro lado, McMahon resolvió un problema electrostático en tres dimensiones usando elementos tetraédricos y funciones lineales.

\footnotetext{
${ }^{1}$ En [12] se pueden encontrar todas las referencias históricas que se describen a continuación.
} 
- Ingeniería: En 1941, Hrenikoff propuso que el comportamiento elástico de una placa podía ser similar, bajo determinadas condiciones de carga, al de un sistema de una dimensión constituido por varillas y vigas conectadas mediante un número finito de puntos. El problema resultante podría entonces abordarse con métodos computacionales conocidos. McHenry y Newmark, más tarde, refinaron esta idea.

Con la introducción comercial de computadoras de alta velocidad, en los años 50, se reformularon de manera matricial los trabajos, análisis y procedimientos realizados hasta entonces, para conseguir una computación automática y eficiente. En este campo destacaron Langefors en 1952 y Argyris en 1954-55. En 1956 Turner, Clough, Martin y Topp dieron un paso más modelando el ala de un avión de alta velocidad mediante el ensamblaje de pequeños paneles de forma triangular.

Los ingenieros reconocieron rápidamente una nueva, potente y práctica herramienta, marcando el comienzo del constante crecimiento del MEF. En la siguiente década se produjo una profusión de escritos acerca de aplicaciones ingenieriles prácticas en los campos de Ingeniería Civil y Aeroespacial. El nombre de MEF apareció, por primera vez, en 1960 en unos escritos de Clough.

Los trabajos de Melosh y Besseling en 1963 y Jones y Veubeke en 1964 mostraron que el MEF podía ser identificado como una forma del principio variacional de Ritz utilizando funciones elementales. En 1965 Zienkiewicz y Cheung ampliaron el campo de aplicación del MEF al demostrar que era aplicable a todo problema que se pudiese expresar de forma variacional.

Resumiendo, el desarrollo del MEF se puede dividir en tres grandes bloques:

- Hasta 1955 se genera el concepto de Elemento Finito.

- Desde 1955 a 1965 se formaliza el concepto de Elemento Finito con numerosas aplicaciones estructurales.

- A partir de 1965 madura esta técnica y se aplica a muchos otros campos. Hasta 1976 aparecen unas 7000 publicaciones. Los matemáticos comienzan a establecer el método sobre firmes fundamentos, con pruebas rigurosas de convergencia, errores y estabilidad. Los algoritmos numéricos se refinan, estableciendo las ecuaciones de los elementos finitos en forma de banda. Se desarrollan generadores de mallas automáticos, gráficos interactivos y otras capacidades de pre y post-proceso. 


\section{2. $\quad$ El Método de los Elementos Finitos (MEF)}

En esta sección se hace una breve descripción del MEF. Para un estudio más profundo ver [12] y [7].

\subsubsection{Definición del Método}

El MEF es una técnica matemática para obtener soluciones numéricas aproximadas de ecuaciones en derivadas parciales. Los pasos seguidos por el MEF para resolver un problema ${ }^{2}$ son:

1. El dominio se divide en un número finito de partes, elementos, cuyo comportamiento se especifica mediante un número finito de parámetros asociados a ciertos puntos característicos denominados nodos. Estos nodos son los puntos de unión de cada elemento con sus adyacentes.

2. La solución del sistema completo sigue las reglas de los problemas discretos, ensamblándose las matrices de todos los elementos.

3. Las incógnitas del problema dejan de ser funciones matemáticas y pasan a ser valores escalares o vectoriales de estas funciones en los nodos.

4. El comportamiento en el interior de cada elemento queda definido a partir del comportamiento de los nodos mediante las denominadas funciones de forma o funciones de interpolación.

En resumen, el MEF se basa en transformar un cuerpo de naturaleza continua en un modelo discreto aproximado: discretización del modelo. El conocimiento de lo que sucede en el interior de este cuerpo aproximado se obtiene mediante la interpolación de los valores conocidos en los nodos. Es, por tanto, una aproximación de los valores de una función a partir del conocimiento de un número determinado y finito de puntos.

\subsubsection{Aplicación del Método}

Como se ha dicho antes, los aspectos prácticos del MEF se han desarrollado en el seno de la Mecánica Estructural. Por esto, la forma más intuitiva de comprender el método, a la vez que la más usada, es aplicándolo a una placa sometida a tensión plana.

\footnotetext{
${ }^{2}$ Debido a su evolución en el seno de la Mecánica Estructural, la mayor parte de la terminología usada está en el contexto de este área.
} 
Sea un elemento finito $e$ definido por sus nodos $\left(i=1, \ldots, N_{n}\right)$ y por su contorno. Los desplazamientos $\mathbf{u}$ de cualquier punto del elemento se aproximan por

$$
\mathbf{u} \approx \mathbf{N} \mathbf{a}=\sum_{i=1}^{N_{n}} N_{i} \mathbf{a}_{i}^{e}
$$

donde $N_{i}$ son las funciones de forma y $\mathbf{a}_{i}^{e}$ son los desplazamientos nodales ${ }^{3}$ del elemento e considerado.

Conocidos los desplazamientos en todos los puntos del elemento, se pueden determinar las deformaciones con ayuda de las relaciones cinemáticas o de compatibilidad. Para el caso estructural

$$
\varepsilon=\varepsilon_{\nabla} \mathbf{u},
$$

donde $\varepsilon_{\nabla}$ es un operador lineal adecuado. Si se sustituye (3.1) en (3.2), se obtiene

$$
\varepsilon=\mathbf{B} \mathbf{a}
$$

donde

$$
\mathbf{B}=\varepsilon_{\nabla} \mathbf{N}
$$

Una vez determinadas las deformaciones en todo punto, con ayuda de la ecuación constitutiva (2.5) se pueden obtener las tensiones.

Se definen las fuerzas nodales (q) como las fuerzas que actúan sobre los nodos y que son estáticamente equivalentes a las tensiones en el contorno y a las fuerzas volumétricas que actúan sobre el elemento. Hay que tener en cuenta que las fuerzas nodales tienen que tener tantas componentes como los desplazamientos nodales. Haciendo uso de la ecuación de equilibrio mecánico

$$
\nabla \cdot \sigma+b=0
$$

se obtiene

$$
\mathbf{q}^{e}=\int_{\Omega^{e}} \mathbf{B}^{T} \sigma d \Omega-\int_{\Omega^{e}} \mathbf{N}^{T} b d \Omega
$$

donde $\sigma$ denota a las tensiones, $\mathbf{b}$ a las fuerzas por unidad de volumen y el superíndice $e$ indica que es a nivel de elemento. Esta relación entre fuerzas nodales, tensiones en el contorno y fuerzas distribuidas se determina mediante el principio de los trabajos virtuales (ver [12] para demostración) y es válida con carácter general, independientemente de las

\footnotetext{
${ }^{3}$ Los desplazamientos nodales se notan como matrices de una columna ya que tienen tantas componentes como grados de libertad, a veces de diferente naturaleza, tenga cada nodo.
} 
relaciones constitutivas entre tensiones y deformaciones. Si las tensiones obedecen a una ley lineal, como la relación constitutiva de la ecuación (2.5), la ecuación (3.6) se puede reescribir como

$$
\mathbf{q}^{e}=\mathbf{K}^{e} \mathbf{a}^{e}+\mathbf{f}^{e}
$$

donde

$$
\mathbf{K}^{e}=\int_{\Omega^{e}} \mathbf{B}^{T} \mathbf{C ~ B} d \Omega \quad ; \quad \mathbf{f}^{\mathbf{e}}=-\int_{\Omega^{e}} \mathbf{N}^{T} \mathbf{b} d \Omega
$$

siendo $\mathbf{C}$ la matriz elástica constitutiva de (2.5), $\mathbf{K}^{e}$ la matriz de rigidez y $\mathbf{f}^{e}$ las fuerzas externas.

\subsubsection{Otros Detalles}

\section{Funciones de Forma}

Las funciones de forma o de interpolación tienen que valer la unidad en los nodos en los que están asociadas y ser nulas en el resto. Además, han de cumplir las siguientes propiedades:

- Derivabilidad: Si el operador $\varepsilon_{\nabla}$ es de orden $m$, la función de forma deberá ser $m$-veces derivable.

- Integrabilidad: Una vez realizada la $m$-ésima derivada, la función de forma tendrá que ser integrable.

- Completitud: Si la función de forma escogida es polinómica (lo más habitual) para que la función se aproxime hasta el término $m$-ésimo a la solución real, el polinomio debe ser completo.

\section{Estimación del Error}

Las posibles fuentes de error cuando se emplea el MEF son muy diversas y se pueden clasificar en:

- Errores de modelización

- En la modelización de las cargas exteriores.

- En la modelización de las condiciones de contorno.

- Propiedades de los materiales.

- Errores de discretización 
- Al aproximar geometrías complejas mediante elementos. Estos errores se corrigen refinando la malla.

- Como norma general, hay que usar elementos pequeños en las zonas donde haya variación de la magnitud que estamos calculando y grandes donde las variaciones sean más suaves. Además, no puede haber saltos bruscos entre elementos, sino que se requiere cierta progresividad.

- Errores de computación

- En la integración numérica.

- En la resolución del sistema de ecuaciones.

\subsubsection{Funcionamiento de un Programa de Elementos Finitos}

Un programa para cálculo mediante MEF tiene que disponer de tres módulos de trabajo:

- Pre-Procesador: Se prepara el modelo para el cálculo, siguiendo los siguientes pasos:

- Geometría del modelo.

- Mallado del modelo.

- Selección del tipo de elemento o elementos.

- Selección del material o materiales.

- Aplicación de las cargas exteriores.

- Aplicación de las condiciones de contorno.

- Calculador: Es la parte del programa que realiza todo el cálculo del MEF y genera las soluciones. En este módulo se siguen los siguientes pasos:

- Se transfieren las cargas al modelo.

- Se generan las matrices de rigidez.

- Se resuelve la triangulación de la matriz.

- Se resuelve el sistema de ecuaciones.

- Se obtiene la solución.

- Post-Procesador: Es la herramienta que permite la representación gráfica de los resultados y donde se realizan los cálculos indirectos. 


\subsubsection{Programas de Elementos Finitos utilizados}

En este trabajo se han usado dos programas de elementos finitos diferentes, FEAP y Ansys.

- FEAP (Finite Element Analysis Program) es un programa de elementos finitos de propósito general diseñado por el profesor Robert L. Taylor, de la Universidad de Califonia, Berkeley, para uso en investigación y educación. El código fuente del programa (escrito en lenguaje FORTRAN) está disponible para compilarse en Windows, sistemas basados en UNIX (como Linux) y Mac OS X. Este código es muy transparente y relativamente fácil de entender, lo que lo convierte en una herramienta ideal para investigadores, ya que además permite crear tus propios elementos. Entre sus deventajas está el no disponer de interfaz gráfica con menús, la dificultad de uso, y el no disponer de forma nativa de elementos para simular placas multicapa, lo que ha obligado a usar otro software que sí lo tiene implementado.

- Ansys es un programa de elementos finitos de propósito general desarrolado por la empresa americana $A N S Y S$, Inc.. Es un software comercial, que dispone de una completa interfaz gráfica y multitud de elementos ya programados. Funciona en Windows y Linux. Sin embargo, no hay acceso al código fuente, por lo que no es posible cambiar los elementos existentes ni añadirle nuevos. Se puede usar sin interfaz gráfica, lo que, si bien añade dificultad de uso (igualando o incluso superando a FEAP en este aspecto) resulta recomendable debido a la rapidez que se gana a la hora de modificar parámetros y la versatilidad a la hora de obtener los resultados del programa. El uso de este software ha sido posible gracias a una colaboración con el Departamento de Mecánica de Estructuras e Ingeniería Hidráulica de la Universidad de Granada. 



\section{Capítulo 4}

\section{Placa unidireccional}

En este capítulo se comparan los resultados obtenidos mediante simulación numérica con resultados teóricos, en el caso particular de una placa unidireccional. Los resultados se dividen en simulaciones con cargas estáticas y con cargas dinámicas.

\subsection{Cargas estáticas}

En primer lugar se presentan casos estáticos de carga. Para ello, se somete la placa a diversos esfuerzos y se obtienen las deformaciones producidas, comparando las obtenidas con el MEF y las teóricas, calculadas implementando las fórmulas correpondientes del Capítulo 2 en GNU Octave, programa libre (bajo licencia GPL) de cálculo computacional que usa un lenguaje similar al conocido programa comercial Matlab.

En todos los casos de cargas estáticas se ha usado una placa de material compuesto tipo T300/5208 Carbon/Epoxy, con las siguientes propiedades:

\begin{tabular}{|c|c|c|c|c|c|c|c|}
\hline$E_{1}(\mathrm{~Pa})$ & $E_{2}(\mathrm{~Pa})$ & $\nu_{12}$ & $G_{12}(\mathrm{~Pa})$ & $\rho\left(\mathrm{kg} / \mathrm{m}^{3}\right)$ & $h(\mathrm{~m})$ & $a(\mathrm{~m})$ & $b(\mathrm{~m})$ \\
\hline $181 \times 10^{9}$ & $10.3 \times 10^{9}$ & 0.28 & $7.17 \times 10^{9}$ & 1600 & $10^{-3}$ & 1 & 1 \\
\hline
\end{tabular}

donde $E_{1}$ es el módulo de Young en la dirección de las fibras, $E_{2}$ el módulo de Young en la direcciones perpendiculares a las fibras, $\nu_{12}$ el coeficiente de Poisson, $G_{12}$ el módulo cortante, $\rho$ la densidad, y $h, a$ y $b$ el espesor, el ancho y el largo de la placa. Se considera que la placa está libre de condiciones de contorno en todos los casos.

\subsubsection{Tracción}

El primer caso que hemos estudiado consiste en someter la placa a esfuerzos de tracción. Un ejemplo de este caso se puede ver en la Figura 4.1, donde podemos ver la placa original (en rojo), la placa tras la deformación (en verde) y la dirección de la fuerza inicialmente 
aplicada (en azul). En la Tabla 4.1 se pueden observar los resultados tantos teóricos como de simulación con FEAP de las deformaciones unitarias $\epsilon_{x}, \epsilon_{y}$ y $\epsilon_{s}$ al aplicar una fuerza de $100 \mathrm{~N}$ a ambos lados de la placa en la dirección del eje $x$, como se ve en la Figura 4.1. Los valores teóricos se han obtenido mediante la ecuacion matricial (2.43). Se puede observar que los resultados de la simulación son completamente satisfactorios, asi como el hecho de que, tal y como se comentaba al final de la sección 2.1.3, en las placas con fibras a cero y noventa grados $\epsilon_{s}$ es igual a cero, pero en las placas con fibras a otros ángulos no.

\begin{tabular}{|c|c|c|c|c|}
\hline & \multicolumn{2}{|c|}{ [0] } & \multicolumn{2}{|c|}{ [36] } \\
\hline & Teoría & FEAP & Teoría & FEAP \\
\hline$\epsilon_{x}$ & $5.5249 \mathrm{e}-07$ & $5.5248 \mathrm{e}-07$ & $4.4794 \mathrm{e}-06$ & $4.4794 \mathrm{e}-06$ \\
\hline$\epsilon_{y}$ & $-1.5470 \mathrm{e}-07$ & $-1.5470 \mathrm{e}-07$ & $-9.1819 \mathrm{e}-07$ & $-9.1817 \mathrm{e}-07$ \\
\hline \multirow[t]{3}{*}{$\epsilon_{s}$} & 0 & 0 & $-4.8502 \mathrm{e}-06$ & $-4.8502 \mathrm{e}-06$ \\
\hline & \multicolumn{2}{|c|}{445} & \multicolumn{2}{|c|}{ [90] } \\
\hline & Teoría & FEAP & Teoría & FEAP \\
\hline$\epsilon_{x}$ & $5.9747 \mathrm{e}-06$ & $5.9747 \mathrm{e}-06$ & $9.7087 \mathrm{e}-06$ & $9.7088 \mathrm{e}-06$ \\
\hline$\overline{\epsilon_{y}}$ & $-9.9879 \mathrm{e}-07$ & $-9.9879 \mathrm{e}-07$ & $-1.5470 \mathrm{e}-07$ & $-1.5470 \mathrm{e}-07$ \\
\hline$\epsilon_{s}$ & $-4.5781 \mathrm{e}-06$ & $-4.5781 \mathrm{e}-06$ & 0 & 0 \\
\hline
\end{tabular}

Tabla 4.1: Deformación de una placa sometida a un esfuerzo estático de tracción. Comparación de los resultados teóricos y de simulación con FEAP

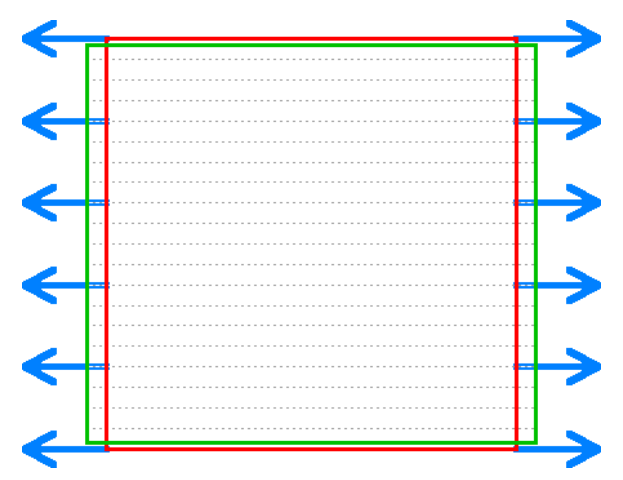

Figura 4.1: Ejemplo de placa [0] sometida a un esfuerzo estático de tracción

\subsubsection{Compresión}

El segundo caso que se presenta consiste en someter la placa a esfuerzos estáticos de compresión, tal y como se muestra en la Figura 4.2, con el mismo código de colores de la Figura 4.1. En la Tabla 4.2 se pueden observar los resultados tantos teóricos como de 
simulación con FEAP de las deformaciones unitarias $\epsilon_{x}, \epsilon_{y}$ y $\epsilon_{s}$ al aplicar una fuerza de $100 \mathrm{~N}$ a ambos lados de la placa en la dirección del eje $x$, como se ve en la Figura 4.2. Se puede observar que los resultados son exactamente iguales a los de la Tabla 4.1, pero de signo contrario.

\begin{tabular}{|c|c|c|c|c|}
\hline & \multicolumn{2}{|c|}{$[0]$} & \multicolumn{2}{|c|}{$[36]$} \\
\hline & Teoría & FEAP & Teoría & FEAP \\
\hline$\epsilon_{x}$ & $-5.5249 \mathrm{e}-07$ & $-5.5248 \mathrm{e}-07$ & $-4.4794 \mathrm{e}-06$ & $-4.4794 \mathrm{e}-06$ \\
\hline$\overline{\epsilon_{y}}$ & $1.5470 \mathrm{e}-07$ & $1.5470 \mathrm{e}-07$ & $9.1819 \mathrm{e}-07$ & $9.1819 \mathrm{e}-07$ \\
\hline$\epsilon_{s}$ & 0 & 0 & $4.8502 \mathrm{e}-06$ & $4.8502 \mathrm{e}-06$ \\
\hline & \multicolumn{2}{|c|}{ 45] } & \multicolumn{2}{|c|}{ [90] } \\
\hline & Teoría & FEAP & Teoría & FEAP \\
\hline$\epsilon_{x}$ & $-5.9747 \mathrm{e}-06$ & $-5.9747 \mathrm{e}-06$ & $-9.7087 \mathrm{e}-06$ & $-9.7088 \mathrm{e}-06$ \\
\hline$\overline{\epsilon_{y}}$ & $9.9879 \mathrm{e}-07$ & $9.9881 \mathrm{e}-07$ & $1.5470 \mathrm{e}-07$ & $1.5470 \mathrm{e}-07$ \\
\hline$\epsilon_{s}$ & $4.5781 \mathrm{e}-06$ & $4.5781 \mathrm{e}-06$ & 0 & 0 \\
\hline
\end{tabular}

Tabla 4.2: Deformación de una placa sometida a un esfuerzo estático de compresión. Comparación de los resultados teóricos y de simulación con FEAP

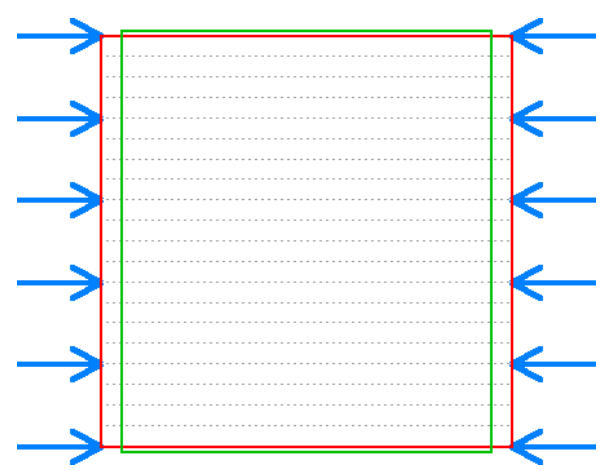

Figura 4.2: Ejemplo de placa [0] sometida a un esfuerzo estático de compresión

\subsubsection{Cortante}

En el tercer caso la placa se ha sometido a esfuerzos estáticos cortantes. Un ejemplo de este tipo de esfuerzos y sus efectos se puede ver en la Figura 4.3, con el mismo código de colores de la Figura 4.1. En la Tabla 4.3 se pueden observar los resultados tantos teóricos como de simulación con FEAP de las deformaciones unitarias $\epsilon_{x}, \epsilon_{y}$ y $\epsilon_{s}$ al aplicar una fuerza de $100 \mathrm{~N}$ a los cuatro lados de la placa, de forma similar a la Figura 4.3. Se puede observar que los resultados de la simulación son completamente satisfactorios, asi como el 
hecho de que, tal y como se comentaba al final de la sección 2.1.3, en las placas con fibras a cero y noventa $\epsilon_{x}$ y $\epsilon_{y}$ son iguales a cero y la placa no sufre cambio en sus dimensiones, pero en las placas con fibras a otros ángulos esto no es así.

\begin{tabular}{|c|c|c|c|c|}
\hline & \multicolumn{2}{|c|}{ [0] } & \multicolumn{2}{|c|}{ [36] } \\
\hline & Teoría & FEAP & Teoría & FEAP \\
\hline$\overline{\epsilon_{x}}$ & 0 & 0 & $-4.8502 \mathrm{e}-06$ & $-4.8502 \mathrm{e}-06$ \\
\hline$\epsilon_{y}$ & 0 & 0 & $-3.8579 \mathrm{e}-06$ & $-3.8580 \mathrm{e}-06$ \\
\hline \multirow[t]{3}{*}{$\epsilon_{s}$} & $1.3947 \mathrm{e}-05$ & $1.3947 \mathrm{e}-05$ & $1.0893 \mathrm{e}-05$ & $1.0893 \mathrm{e}-05$ \\
\hline & \multicolumn{2}{|c|}{ [45] } & \multicolumn{2}{|c|}{ [90] } \\
\hline & Teoría & FEAP & Teoría & FEAP \\
\hline$\epsilon_{x}$ & $-4.5781 \mathrm{e}-06$ & $-4.5781 \mathrm{e}-06$ & 0 & 0 \\
\hline$\overline{\epsilon_{y}}$ & $-4.5781 \mathrm{e}-06$ & $-4.5782 \mathrm{e}-06$ & 0 & 0 \\
\hline$\epsilon_{s}$ & $1.0571 \mathrm{e}-05$ & $1.0571 \mathrm{e}-05$ & $1.3947 \mathrm{e}-05$ & $1.3947 \mathrm{e}-05$ \\
\hline
\end{tabular}

Tabla 4.3: Deformación de una placa sometida a un esfuerzo estático cortante. Comparación de los resultados teóricos y de simulación con FEAP

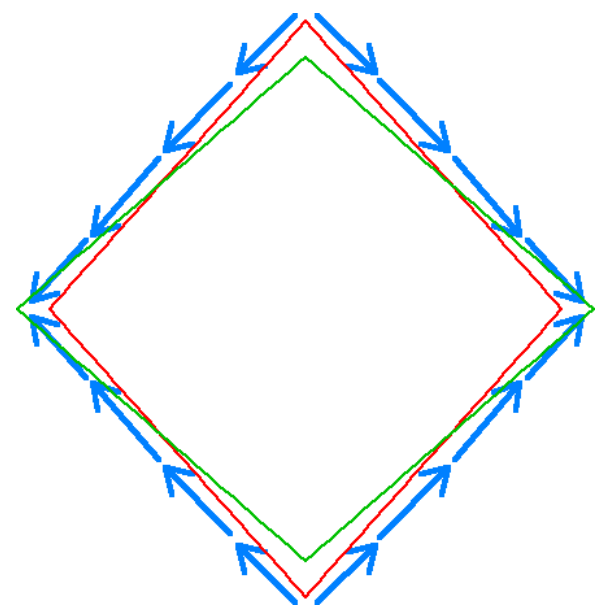

Figura 4.3: Ejemplo de placa [0] sometida a un esfuerzo estático cortante 


\subsection{Cargas dinámicas}

En esta sección se analiza la validez de las simulaciones mediante el MEF para placas ortótropas unidireccionales sometidas a cargas dinámicas. Se han realizado dos tipos de análisis:

- Análisis modal: Cálculo de las frecuencias y modos propios de vibración de la placa, comparando los resultados teóricos con los de simulación mediante los programas FEAP y Ansys, usando en ambos programas un mallado cuadrado de 36x36 elementos. El elemento usado fue de tipo Shell, y viene implementado de forma nativa en ambos programas.

- Análisis transitorio: Cálculo de la respuesta temporal de vibración en cualquier punto de la placa sometida a cargas transitorias puntuales. En este caso se comparan los resultados numéricos obtenidos con FEAP con los resultados teóricos. El mallado usado en FEAP ha sido de 48x48, y el elemento de tipo Shell, al igual que en el caso anterior.

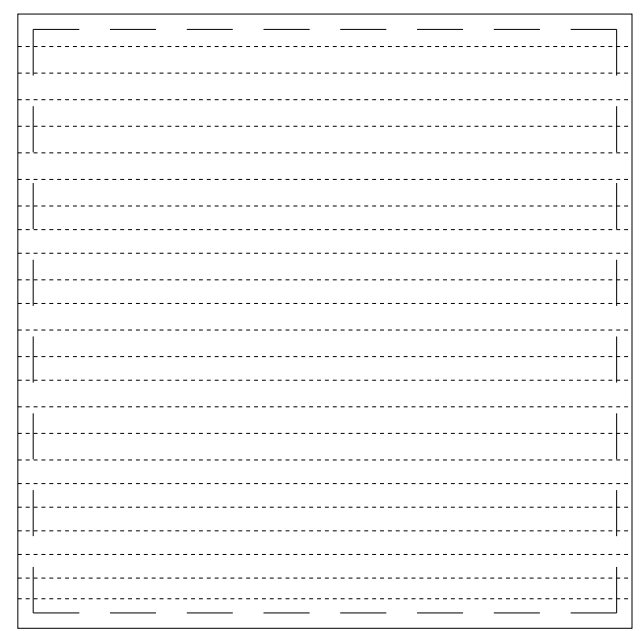

Figura 4.4: Ejemplo de placa [0] simplemente apoyada

\subsubsection{Vibraciones naturales: Análisis modal}

\section{a) Placa $[0]$ s-s-s-s}

En primer lugar se presenta el caso de una placa [0] cuadrada ortótropa, simplemente apoyada en sus cuatro lados, véase la Figura 4.4. Esto es, impedimos el desplazamiento en los cuatro lados de la placa, pero no los giros. La placa en cuestión tiene las siguientes propiedades: 


\begin{tabular}{|c|c|c|c|c|c|c|c|}
\hline$E_{1}(\mathrm{~Pa})$ & $E_{2}(\mathrm{~Pa})$ & $\nu_{12}$ & $G_{12}(\mathrm{~Pa})$ & $\rho\left(\mathrm{kg} / \mathrm{m}^{3}\right)$ & $h(\mathrm{~m})$ & $a(\mathrm{~m})$ & $b(\mathrm{~m})$ \\
\hline $1.2 \times 10^{8}$ & $1.2 \times 10^{7}$ & 0.513 & $3 \times 10^{6}$ & 1000 & $10^{-2}$ & 1 & 1 \\
\hline
\end{tabular}

Se han calculado las 32 primeras frecuencias propias haciendo uso de la expresión (2.50), y se han comparado con las obtenidas mediante simulación numérica con los programas FEAP y Ansys. Los resultados se pueden ver en la Tabla 4.4. Se observa que los resultados numéricos son bastante satisfactorios, siendo en general los de FEAP algo mejores que los de Ansys. También se puede observar que entre los modos 18 y 30 Ansys introduce una frecuencia más que FEAP y la teoría, posiblemente la frecuencia $f_{24}$. Este es un hecho a tener en cuenta a la hora de identificar modos de vibración, la simulación en alguno de los programas usados puede introducir módos espúreos, o bien, no proporcionar algunos modos que existan en la realidad.

\begin{tabular}{|c|c|c|c|}
\hline & Teoría & FEAP & Ansys \\
\hline$f_{1}$ & 1.81 & 1.81 & 1.81 \\
\hline$f_{2}$ & 2.94 & 2.93 & 2.93 \\
\hline$f_{3}$ & 5.26 & 5.25 & 5.24 \\
\hline$f_{4}$ & 6.54 & 6.54 & 6.49 \\
\hline$f_{5}$ & 7.26 & 7.24 & 7.20 \\
\hline$f_{6}$ & 8.69 & 8.67 & 8.65 \\
\hline$f_{7}$ & 8.91 & 8.86 & 8.83 \\
\hline$f_{8}$ & 11.74 & 11.67 & 11.64 \\
\hline$f_{9}$ & 13.18 & 13.14 & 13.09 \\
\hline$f_{10}$ & 14.49 & 14.48 & 14.23 \\
\hline$f_{11}$ & 15.09 & 15.04 & 14.81 \\
\hline$f_{12}$ & 15.80 & 15.69 & 15.65 \\
\hline$f_{13}$ & 16.34 & 16.23 & 16.04 \\
\hline$f_{14}$ & 18.52 & 18.35 & 18.19 \\
\hline$f_{15}$ & 18.69 & 18.63 & 18.52 \\
\hline$f_{16}$ & 21.02 & 20.87 & 20.78 \\
\hline
\end{tabular}

\begin{tabular}{|c|c|c|c|}
\hline & Teoría & FEAP & Ansys \\
\hline$f_{17}$ & 21.86 & 21.59 & 21.47 \\
\hline$f_{18}$ & 25.21 & 25.13 & 24.84 \\
\hline$f_{19}$ & 25.63 & 25.60 & 24.92 \\
\hline$f_{20}$ & 26.18 & 26.05 & 25.36 \\
\hline$f_{21}$ & 26.42 & 26.08 & 25.95 \\
\hline$f_{22}$ & 27.24 & 27.05 & 26.40 \\
\hline$f_{23}$ & 27.36 & 27.14 & 26.98 \\
\hline$f_{24}$ & 29.04 & 28.71 & 27.39 \\
\hline$f_{25}$ & 31.78 & 31.28 & 28.15 \\
\hline$f_{26}$ & 32.21 & 31.72 & 30.82 \\
\hline$f_{27}$ & 32.76 & 32.62 & 31.60 \\
\hline$f_{28}$ & 34.76 & 34.47 & 32.26 \\
\hline$f_{29}$ & 35.62 & 34.93 & 34.18 \\
\hline$f_{30}$ & 39.18 & 38.55 & 34.58 \\
\hline$f_{31}$ & 39.96 & 39.75 & 38.08 \\
\hline$f_{32}$ & 40.48 & 39.89 & 38.37 \\
\hline
\end{tabular}

Tabla 4.4: Frecuencias propias de una placa [0] simplemente apoyada obtenidas teóricamente y con los programas FEAP y Ansys, todos los valores en $\mathrm{Hz}$

\section{b) Placa [0] c-c-c-c}

El segundo ejemplo que se presenta es el de una placa [0] cuadrada ortótropa, empotrada en sus cuatro lados, esto es, impedimos tanto los desplazamientos como los giros, como se puede ver en la Figura 4.5. Las propiedades de la placa son:

\begin{tabular}{|c|c|c|c|c|c|c|c|}
\hline$E_{1}(\mathrm{~Pa})$ & $E_{2}(\mathrm{~Pa})$ & $\nu_{12}$ & $G_{12}(\mathrm{~Pa})$ & $\rho\left(\mathrm{kg} / \mathrm{m}^{3}\right)$ & $h(\mathrm{~m})$ & $a(\mathrm{~m})$ & $b(\mathrm{~m})$ \\
\hline $1.2 \times 10^{8}$ & $1.2 \times 10^{7}$ & 0.722 & $6 \times 10^{6}$ & 1000 & $10^{-2}$ & 1 & 1 \\
\hline
\end{tabular}


$\mathrm{Al}$ igual que en el caso anterior, se han calculado las primeras frecuencias propias teóricamente, haciendo uso de la expresión (2.50), y se han comparado con las obtenidas mediante simulación numérica. Los resultados se pueden ver en la Tabla 4.5. Por brevedad, y como ejemplo, sólo se presentan las frecuencias $f_{1}-f_{6}, f_{10}, f_{20} \mathrm{y}$ $f_{30}$. Al igual que en el caso anterior los resultados son bastante satisfactorios, siendo también en este caso, en general, los resultados de FEAP un poco mejores que los de Ansys.

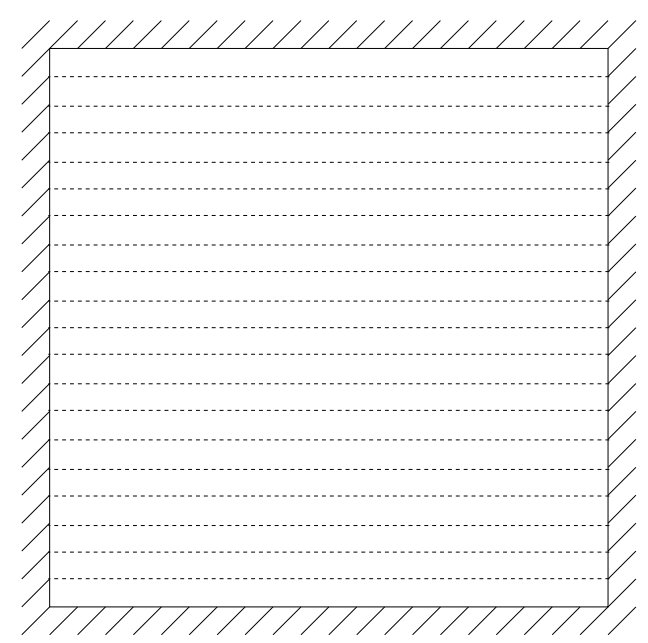

Figura 4.5: Ejemplo de placa [0] empotrada

\begin{tabular}{|c|c|c|c|}
\hline & Teoría & FEAP & Ansys \\
\hline$f_{1}$ & 4.01 & 4.00 & 3.98 \\
\hline$f_{2}$ & 5.35 & 5.33 & 5.32 \\
\hline$f_{3}$ & 7.96 & 7.92 & 7.91 \\
\hline$f_{4}$ & 10.40 & 10.37 & 10.26 \\
\hline$f_{5}$ & 11.43 & 11.37 & 11.29 \\
\hline$f_{6}$ & 11.79 & 11.73 & 11.71 \\
\hline$f_{10}$ & 20.07 & 20.02 & 19.67 \\
\hline$f_{20}$ & 33.67 & 33.23 & 32.86 \\
\hline$f_{30}$ & 47.17 & 46.82 & 46.50 \\
\hline
\end{tabular}

Tabla 4.5: Frecuencias propias de una placa [0] empotrada (en $\mathrm{Hz})$

\section{c) Placa $[0]$ c-c-s-s}

El tercer ejemplo que se presenta es el de una placa de propiedades similares a la anterior pero con dos de sus lados contiguos simplemente apoyados, y los otros dos anclados, como se puede ver en la Figura 4.6. Los resultados para este caso se 


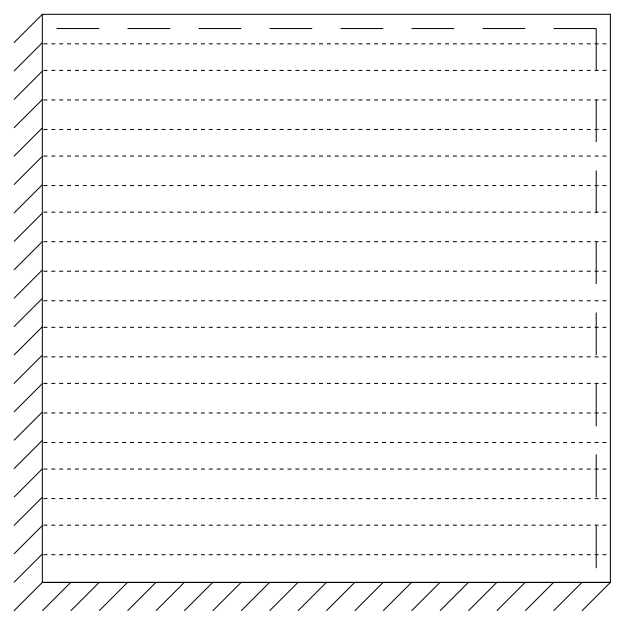

Figura 4.6: Ejemplo de placa [0] empotrada-simplemente apoyada

pueden observar en la Tabla 4.6. Al igual que en los casos anteriores, los resultados son satisfactorios, siendo algo mejores los de FEAP.

\begin{tabular}{|c|c|c|c|}
\hline & Teoría & FEAP & Ansys \\
\hline$f_{1}$ & 2.86 & 2.85 & 2.84 \\
\hline$f_{2}$ & 4.17 & 4.16 & 4.15 \\
\hline$f_{3}$ & 6.71 & 6.68 & 6.67 \\
\hline$f_{4}$ & 8.47 & 8.45 & 8.39 \\
\hline$f_{5}$ & 9.48 & 9.44 & 9.39 \\
\hline$f_{6}$ & 10.40 & 10.35 & 10.33 \\
\hline$f_{10}$ & 17.34 & 17.30 & 17.08 \\
\hline$f_{20}$ & 30.32 & 30.17 & 29.60 \\
\hline$f_{30}$ & 44.67 & 44.29 & 43.30 \\
\hline
\end{tabular}

Tabla 4.6: Frecuencias propias de una placa [0] empotrada-simplemente apoyada (en $\mathrm{Hz}$ )

\section{d) Placa c-c-c-c con diferentes ángulos}

Hasta ahora se han considerado placas con fibras a cero grados respecto al eje X, [0]. Presentamos ahora los resultados obtenidos con placas en los que las fibras están en otras direcciones. Para ello se ha considerado una placa cuadrada empotrada, con las fibras a diferentes ángulos, comparando sus cuatro primeras frecuencias propias, obtenidas teóricamente (usando la ecuacion (2.50)) y numéricamente. Las propiedades de la placa en este caso son las siguientes:

\begin{tabular}{|c|c|c|c|c|c|c|c|}
\hline$E_{1}(\mathrm{~Pa})$ & $E_{2}(\mathrm{~Pa})$ & $\nu_{12}$ & $G_{12}(\mathrm{~Pa})$ & $\rho\left(\mathrm{kg} / \mathrm{m}^{3}\right)$ & $h(\mathrm{~m})$ & $a(\mathrm{~m})$ & $b(\mathrm{~m})$ \\
\hline $10^{11}$ & $10^{10}$ & 0.3 & $2.5 \times 10^{9}$ & 1000 & $10^{-2}$ & 1 & 1 \\
\hline
\end{tabular}


Los resultados se pueden ver en la Tabla 4.7.

\begin{tabular}{|c|c|c|c|c|c|c|}
\hline & \multicolumn{3}{|c|}{ [0] } & \multicolumn{3}{|c|}{ [15] } \\
\hline & Teoría & FEAP & Ansys & Teoría & FEAP & Ansys \\
\hline$f_{1}$ & 110.54 & 110.51 & 109.59 & 106.53 & 106.46 & 105.64 \\
\hline$f_{2}$ & 143.65 & 143.42 & 142.71 & 145.35 & 145.08 & 144.32 \\
\hline$f_{3}$ & 214.02 & 213.57 & 212.87 & 219.74 & 219.22 & 218.23 \\
\hline$f_{4}$ & 289.46 & 289.41 & 283.63 & 274.20 & 274.06 & 269.02 \\
\hline & \multicolumn{3}{|c|}{ [30] } & \multicolumn{3}{|c|}{ [45] } \\
\hline & Teoría & FEAP & Ansys & Teoría & FEAP & Ansys \\
\hline$f_{1}$ & 98.46 & 98.34 & 97.70 & 94.58 & 94.42 & 93.84 \\
\hline$f_{2}$ & 153.01 & 152.62 & 151.61 & 161.45 & 161.10 & 159.81 \\
\hline$f_{3}$ & 233.90 & 233.06 & 231.25 & 217.06 & 215.76 & 213.19 \\
\hline$f_{4}$ & 239.20 & 238.64 & 235.16 & 240.77 & 239.53 & 237.26 \\
\hline
\end{tabular}

Tabla 4.7: Frecuencias propias de una placa cuadrada empotrada con fibras a diferentes ángulos (en $\mathrm{Hz})$

Se ha considerado también una placa similar a la anterior, pero rectangular, sus propiedades son las siguientes:

\begin{tabular}{|c|c|c|c|c|c|c|c|}
\hline$E_{1}(\mathrm{~Pa})$ & $E_{2}(\mathrm{~Pa})$ & $\nu_{12}$ & $G_{12}(\mathrm{~Pa})$ & $\rho\left(\mathrm{kg} / \mathrm{m}^{3}\right)$ & $h(\mathrm{~m})$ & $a(\mathrm{~m})$ & $b(\mathrm{~m})$ \\
\hline $10^{11}$ & $10^{10}$ & 0.3 & $2.5 \times 10^{9}$ & 1000 & $10^{-2}$ & 2 & 1 \\
\hline
\end{tabular}

Los resultados se muestran en la Tabla 4.8. Como puede verse, al igual que en todos los casos estudiados con anterioridad, los resultados con FEAP son ligeramente mejores que los obtenidos con Ansys.

\section{e) Placas [90] y [45] c-c-c-c: modos propios}

En esta sección se presentan los modos propios obtenidos numéricamente comparados con los modos representados de forma esquemática y los resultados experimentales, obtenidos de [11].

La placa considerada es cuadrada, ortótropa, y tiene las siguientes propiedades:

\begin{tabular}{|c|c|c|c|c|c|c|c|}
\hline$E_{1}(\mathrm{~Pa})$ & $E_{2}(\mathrm{~Pa})$ & $\nu_{12}$ & $G_{12}(\mathrm{~Pa})$ & $\rho\left(\frac{k g}{\mathrm{~m}^{3}}\right)$ & $h(\mathrm{~m})$ & $a(\mathrm{~m})$ & $b(\mathrm{~m})$ \\
\hline $213,7 \times 10^{9}$ & $18,6 \times 10^{9}$ & 0.28 & $5,2 \times 10^{9}$ & 2052 & $1,08 \times 10^{-3}$ & 0.30 & 0.30 \\
\hline
\end{tabular}

Se han considerado dos direcciones de la fibras: [90] y [45]. Los resultados se pueden ver en las Tablas 4.9 y 4.10, donde se presentan, por filas, los modos dibujados de 


\begin{tabular}{|c|c|c|c|c|c|c|}
\hline & \multicolumn{3}{|c|}{ [0] } & \multicolumn{3}{|c|}{ [15] } \\
\hline & Teoría & FEAP & Ansys & Teoría & FEAP & Ansys \\
\hline$f_{1}$ & 43.07 & 43.08 & 43.04 & 44.64 & 44.60 & 44.54 \\
\hline$f_{2}$ & 81.21 & 81.15 & 80.81 & 79.27 & 79.15 & 78.86 \\
\hline$f_{3}$ & 96.06 & 96.00 & 95.86 & 101.55 & 101.45 & 101.22 \\
\hline \multirow[t]{4}{*}{$f_{4}$} & 122.16 & 121.83 & 121.67 & 121.93 & 121.62 & 121.23 \\
\hline & & & & & & \\
\hline & \multicolumn{3}{|c|}{$[26,6]$} & \multicolumn{3}{|c|}{ [45] } \\
\hline & Teoría & FEAP & Ansys & Teoría & FEAP & Ansys \\
\hline$f_{1}$ & 48.74 & 48.69 & 48.58 & 64.01 & 63.83 & 63.52 \\
\hline$f_{2}$ & 78.07 & 77.78 & 77.51 & 81.76 & 81.17 & 80.84 \\
\hline$f_{3}$ & 115.79 & 115.48 & 115.00 & 109.98 & 108.71 & 108.29 \\
\hline \multirow[t]{4}{*}{$f_{4}$} & 117.41 & 116.87 & 116.35 & 146.32 & 144.19 & 143.57 \\
\hline & \multirow{2}{*}{\multicolumn{3}{|c|}{$[60]$}} & & & \\
\hline & & & & \multicolumn{3}{|c|}{ [75] } \\
\hline & Teoría & FEAP & Ansys & Teoría & FEAP & Ansys \\
\hline$f_{1}$ & 82.41 & 82.33 & 81.70 & 98.09 & 98.05 & 97.09 \\
\hline$f_{2}$ & 91.58 & 91.05 & 90.54 & 102.93 & 102.62 & 101.85 \\
\hline$f_{3}$ & 109.52 & 108.32 & 107.93 & 113.86 & 113.16 & 112.69 \\
\hline \multirow[t]{4}{*}{$f_{4}$} & 137.19 & 134.89 & 134.56 & 133.46 & 132.00 & 131.84 \\
\hline & \multirow{2}{*}{\multicolumn{3}{|c|}{ [90] }} & & & \\
\hline & & & & & & \\
\hline & Teoría & FEAP & Ansys & & & \\
\hline$f_{1}$ & 104.08 & 104.09 & 102.99 & & & \\
\hline$f_{2}$ & 107.82 & 107.58 & 106.70 & & & \\
\hline$f_{3}$ & 116.67 & 116.11 & 115.57 & & & \\
\hline$f_{4}$ & 133.13 & 132.23 & 132.09 & & & \\
\hline
\end{tabular}

Tabla 4.8: Frecuencias propias de una placa rectangular empotrada con fibras a diferentes ángulos (en $\mathrm{Hz})$

forma esquemática, los experimentales, los que proporciona FEAP y los que proporciona Ansys, acompañados de su frecuencia propia (en el caso del esquemático, la frecuencia analítica correspondiente). Como se puede observar, para ambas placas la correspondencia en modos es bastante satisfactoria, sin embargo no es así en frecuencias, sobre todo las experimentales. Esto puede deberse a la dificultad de hacer experimentos con placas empotradas, y lo rudimentario de éstos. Para más detalles del experimento ver [2]. 


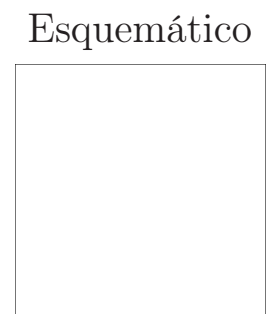

125

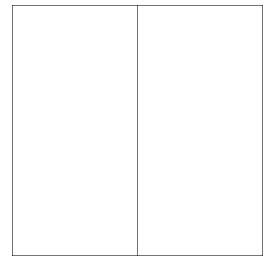

159

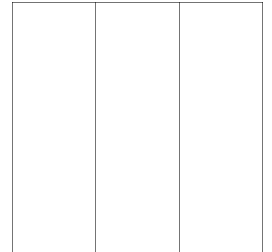

232

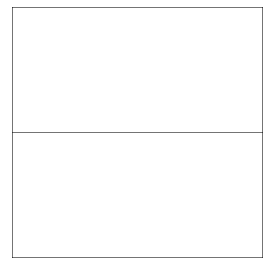

329

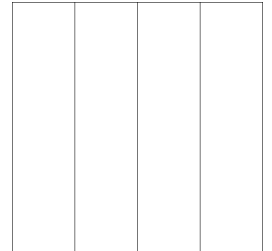

343

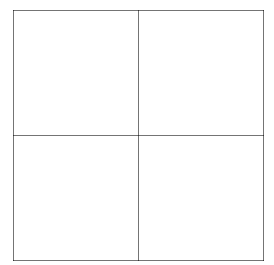

350

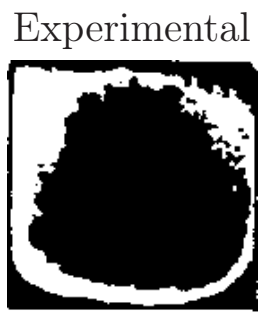

107

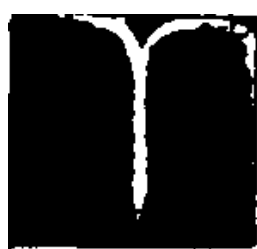

123

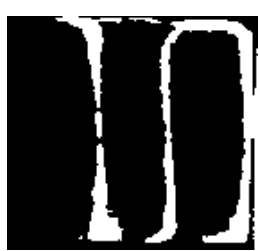

204

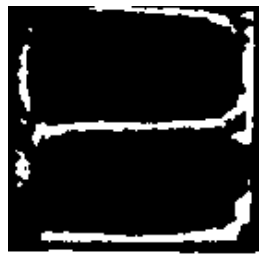

233

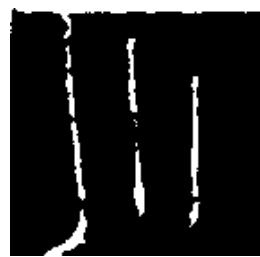

343

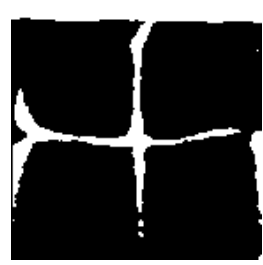

301
FEAP

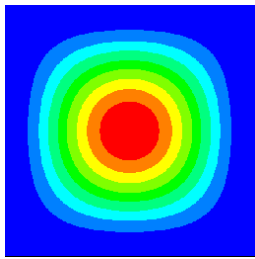

129.6

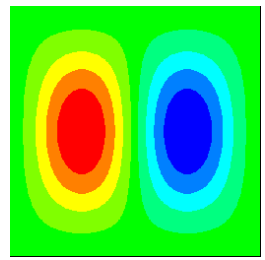

164.5

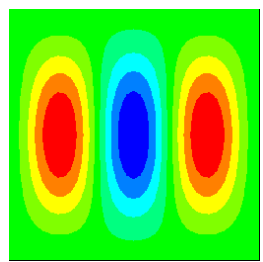

240.1

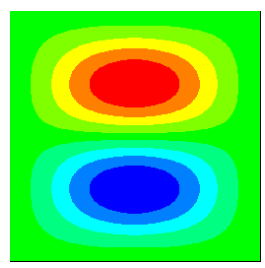

341.5

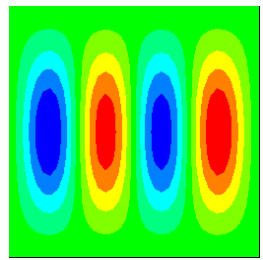

362.2

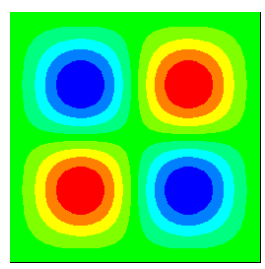

356.0
Ansys

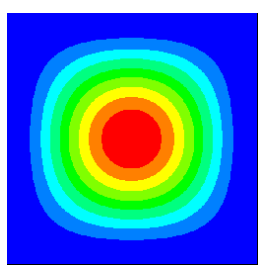

129.6

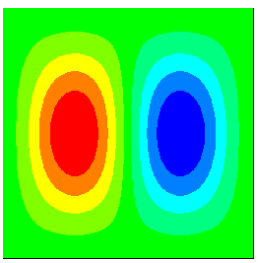

164.6

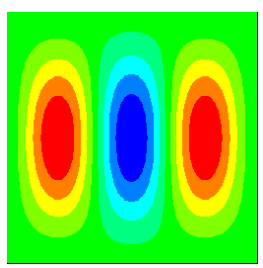

240.5

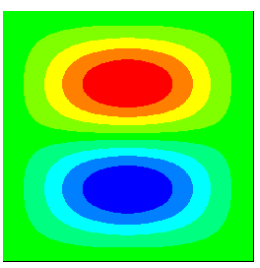

340.6

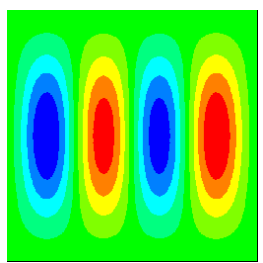

362.1

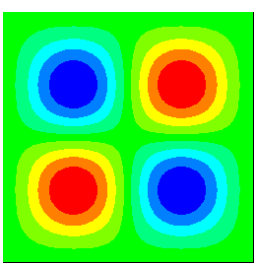

356.5 


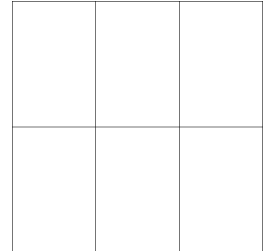

397

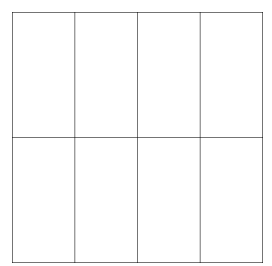

482

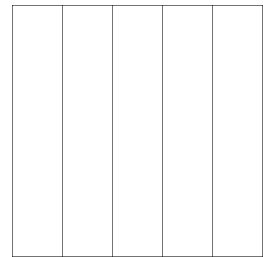

490

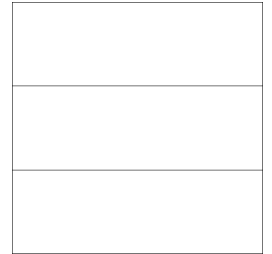

639

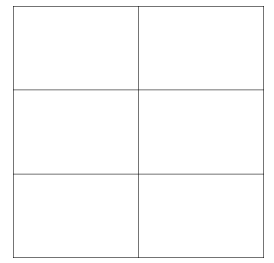

655

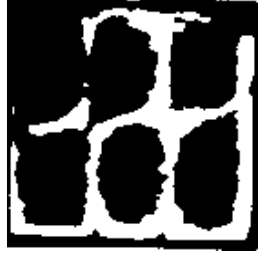

360

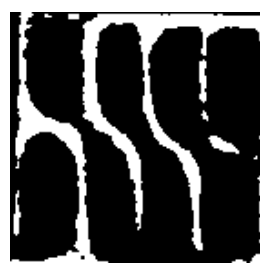

451

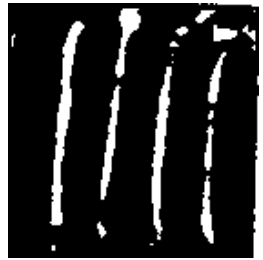

475

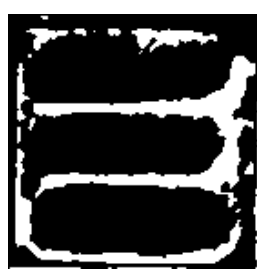

555

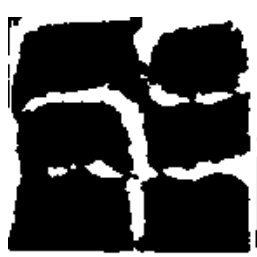

545

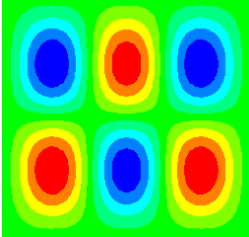

410.6

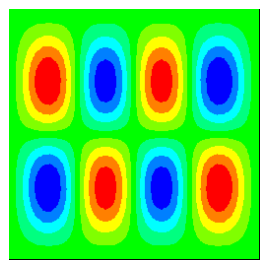

496.8

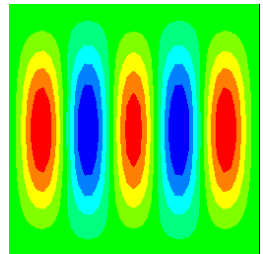

508.3

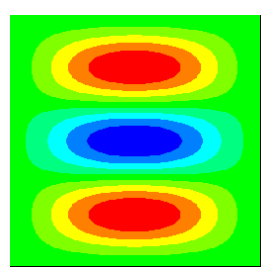

663.6

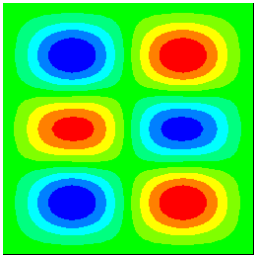

678.9

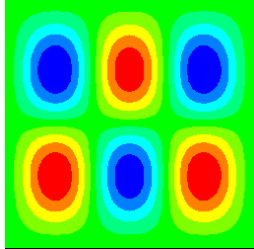

411.7

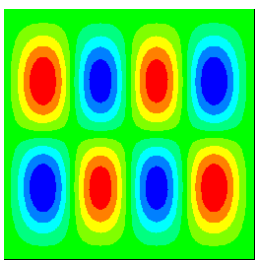

499.2

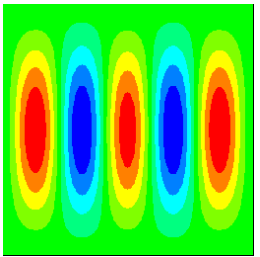

508.9

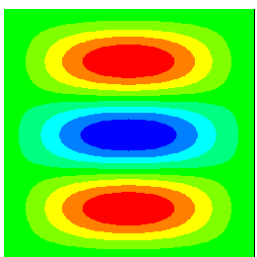

660.2

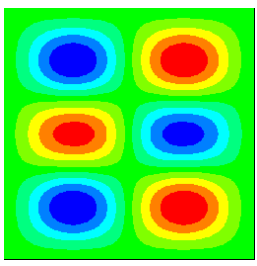

677.1

Tabla 4.9: Modos propios y frecuencias propias (en $\mathrm{Hz}$ ) de una placa [90] cuadrada empotrada 

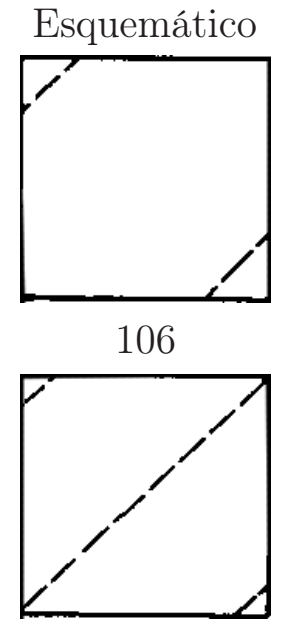

178
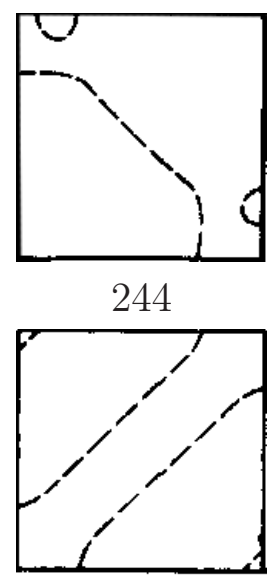

264

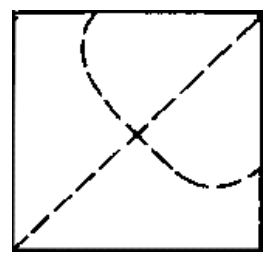

359

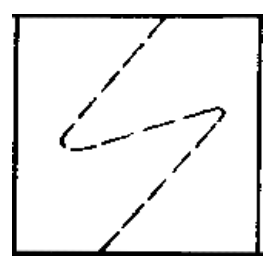

365

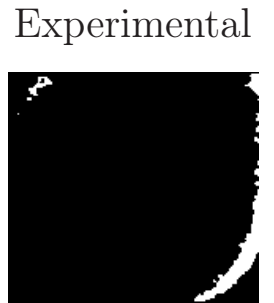

92

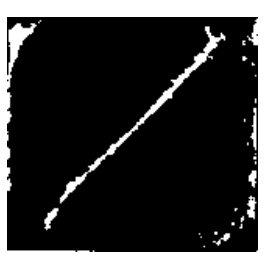

156

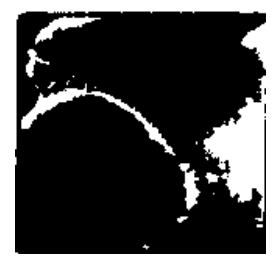

215

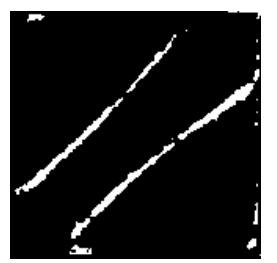

239

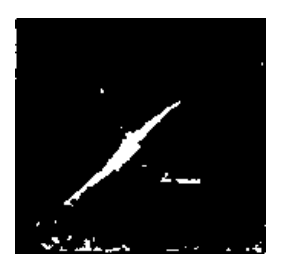

329

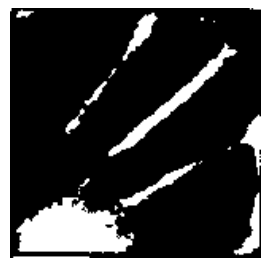

334

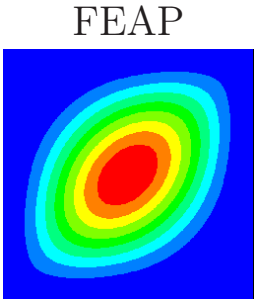

109.4

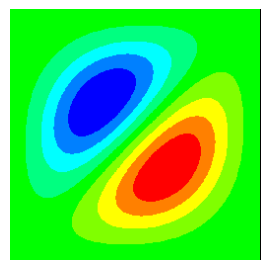

184.7

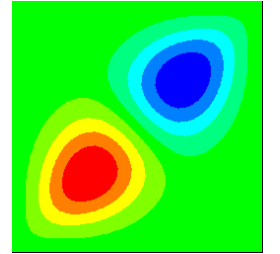

251.4

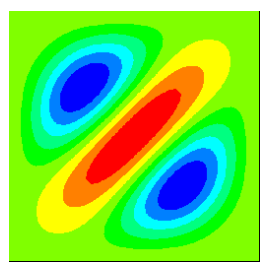

273.0

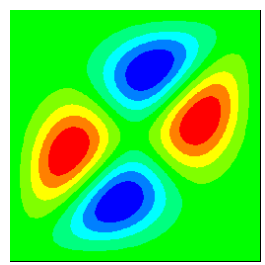

372.0

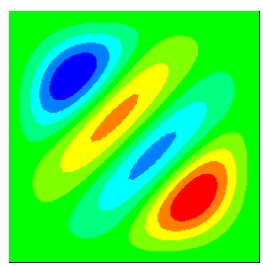

376.7
Ansys

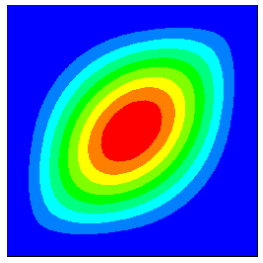

109.37

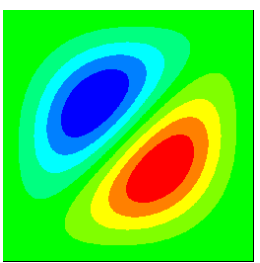

184.67

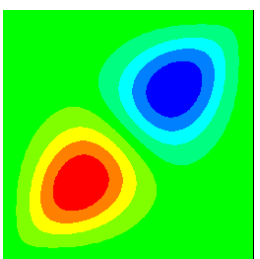

251.28

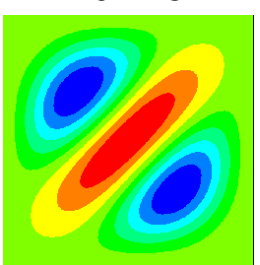

273.08

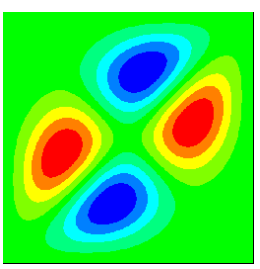

371.38

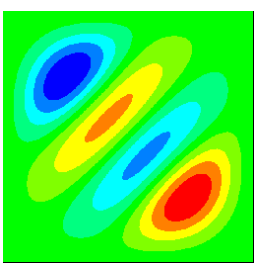

377.07

Tabla 4.10: Modos propios y frecuencias propias (en Hz) de una placa [45] cuadrada empotrada 


\subsubsection{Vibracions forzadas: Análisis transitorio}

Para el análisis de vibraciones forzadas se ha usado la misma placa que en el apartado a) de la sección anterior, es decir, una placa [0] cuadrada ortótropa, simplemente apoyada en sus cuatro lados, como la mostrada en la Figura 4.4, con las siguientes propiedades:

\begin{tabular}{|c|c|c|c|c|c|c|c|}
\hline$E_{1}(\mathrm{~Pa})$ & $E_{2}(\mathrm{~Pa})$ & $\nu_{12}$ & $G_{12}(\mathrm{~Pa})$ & $\rho\left(\mathrm{kg} / \mathrm{m}^{3}\right)$ & $h(\mathrm{~m})$ & $a(\mathrm{~m})$ & $b(\mathrm{~m})$ \\
\hline $1.2 \times 10^{8}$ & $1.2 \times 10^{7}$ & 0.513 & $3 \times 10^{6}$ & 1000 & $10^{-2}$ & 1 & 1 \\
\hline
\end{tabular}

La placa se ha excitado mediante cuatro tipo de fuerzas: ruido blanco gaussiano, pulso gaussiano, seno de baja frecuencia $(2.5 \mathrm{~Hz})$ y seno de alta frecuencia $(40 \mathrm{~Hz})$. En todos las simulaciones el punto de excitación ha sido $(0.417,0.25) \mathrm{m}$ y se ha obtenido la señal de respuesta en dos puntos distintos, $(0.1,0.021) \mathrm{m}$ (punto 1) y $(0.73,0.917) \mathrm{m}$ (punto 2). Puesto que no existe solución analítica, para validar el resultado numérico se ha realizado la Transformada de Fourier y se ha comparado la posición de los picos con los valores de las frecuencias propias de la placa, mostradas en la Tabla 4.4. En todos los casos se ha usado un periodo de muestreo de $0.01 \mathrm{~s}$, y el tiempo total de medida fué de $10 \mathrm{~s}$ (1000 datos), lo que nos da una discretización en el espectro de frecuencias de $0.1 \mathrm{~Hz}$, y un intervalo de 0 a $50 \mathrm{~Hz}$, suficiente para comparar con las frecuencias de la Tabla 4.4. En las Figuras 4.7, 4.8, 4.9 y 4.10 se presentan los cuatro casos estudiados. En cada una de ellas podemos ver la fuerza con la que se ha excitado, y los desplazamientos verticales de la placa en los dos puntos de medida, así como sus respectivas Transformadas de Fourier.

En la Figura 4.7 podemos observar que la Transformada de Fourier de la excitación (ruido blanco gaussiano) es también un ruido blanco gaussiano. Sin embargo en la Transformada de Fourier de la respuesta transitoria de la placa se pueden observar los picos correspondientes a las frecuencias propias de la placa, aunque con un poco de ruido. Se observa también que los dos puntos de medida no nos dan las mismas amplitudes, lo que nos indica la importancia del punto de medida.

En la Figura 4.8 podemos observar que la Transformada de Fourier de un pulso gaussiano es otro pulso gaussiano de anchura inversamente proporcional a la anchura del pulso original. En cuanto a la Transformada de Fourier de la respuesta transitoria de la placa, se observa que este tipo de excitación nos da un espectro más limpio que el ruido gaussiano, y permite una mejor identificación de picos, pero por contra excita con menor amplitud las frecuencias altas. La posición de los picos coincide con gran exactitud con las frecuencias propias de la placa, expuestas en la Tabla 4.4.

En la Figura 4.9 vemos que al excitar con un seno de frecuencia baja sólo se excitan las frecuencias más bajas, observándose, aparte de los picos correspondientes a las frecuencias propias, un pico de $2.5 \mathrm{~Hz}$ correspondiente a la excitación. Sólo se muestra la Transformada 
de Fourier hasta $10 \mathrm{~Hz}$, ya que no se excitan frecuencias más altas. Se observan picos en las posiciones $1.8 \mathrm{~Hz}, 2.9 \mathrm{~Hz}, 5.3 \mathrm{~Hz}, 6.5 \mathrm{~Hz}$ y $8.7 \mathrm{~Hz}$, que coincide exactamente con las frecuencias propias de la placa.

Por último, en la Figura 4.10 vemos que al excitar con un seno de frecuencia alta (del orden del modo 30) se excitan muchas más frecuencias, pero especialmente las cercanas a la frecuencia de excitación. Se puede observar un pico muy energético en $40 \mathrm{~Hz}$, que corresponde a la frecuencia de excitación.
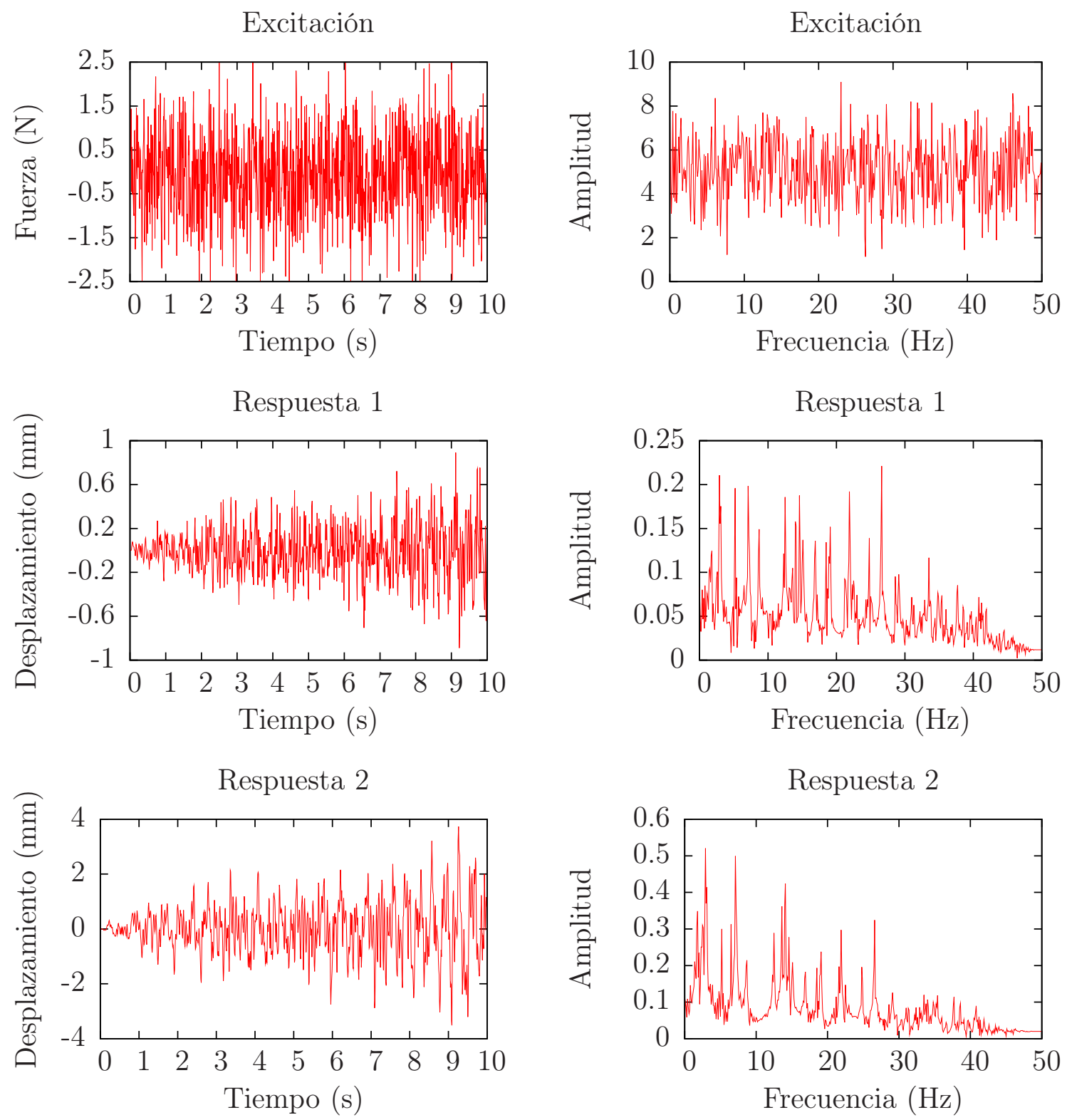

Figura 4.7: Excitación y respuesta en dos puntos de una placa [0] simplemente apoyada en los dominios del tiempo y la frecuencia. Excitación: ruido gaussiano 
Excitación

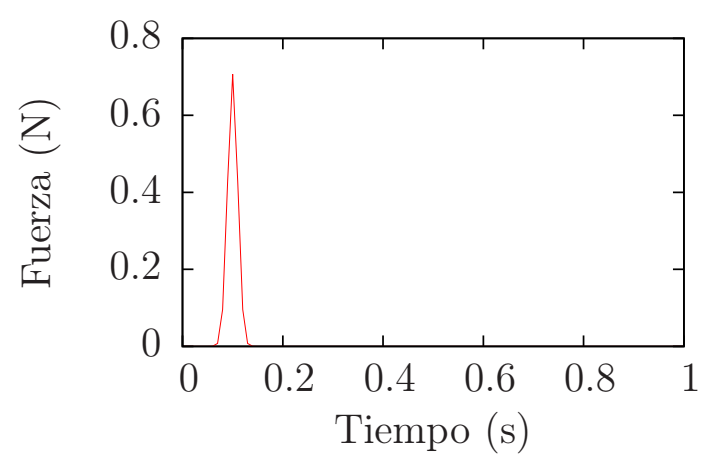

Respuesta 1

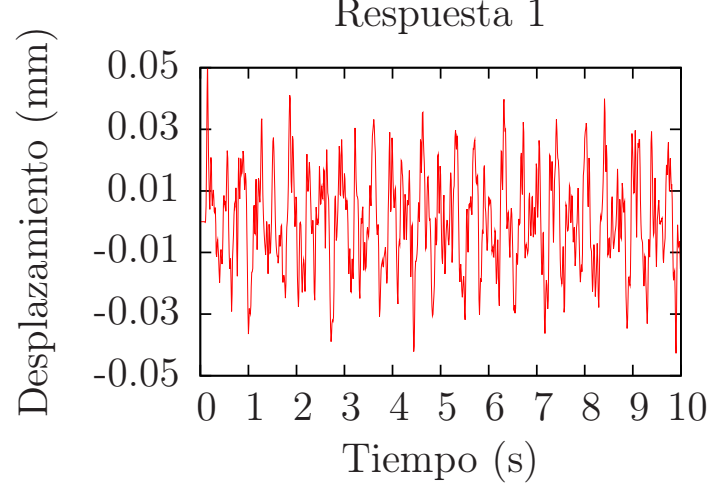

Respuesta 2

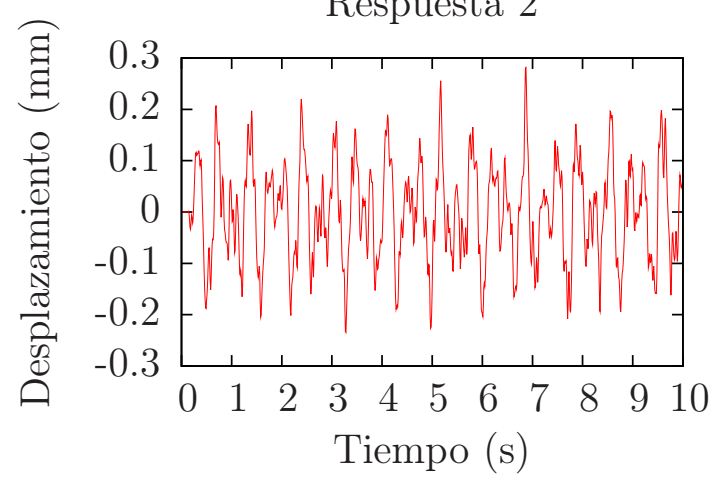

Excitación

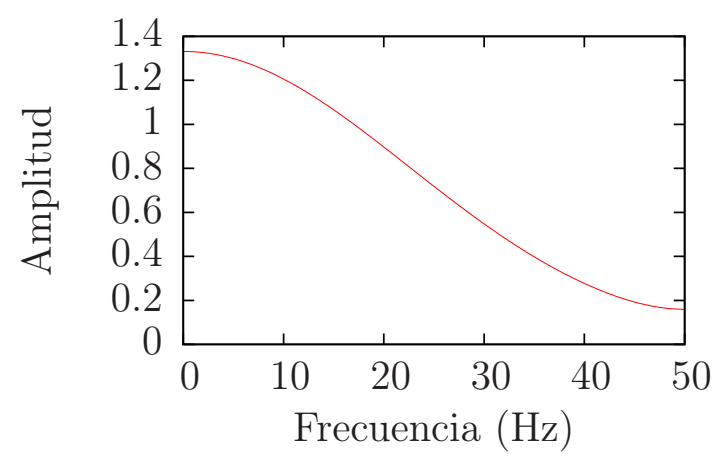

Respuesta 1

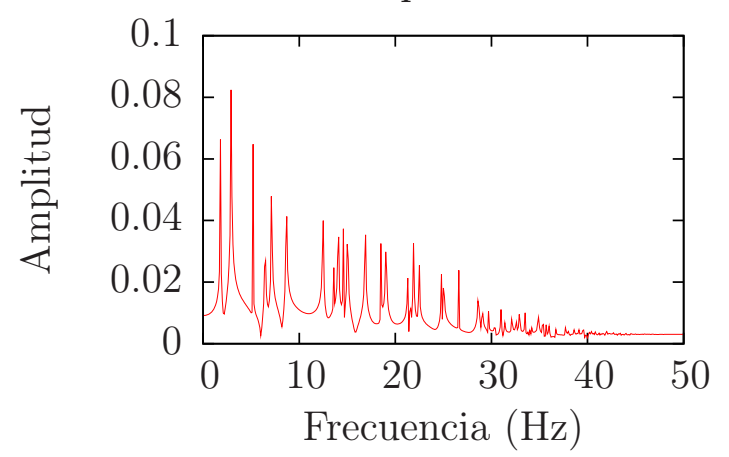

Respuesta 2

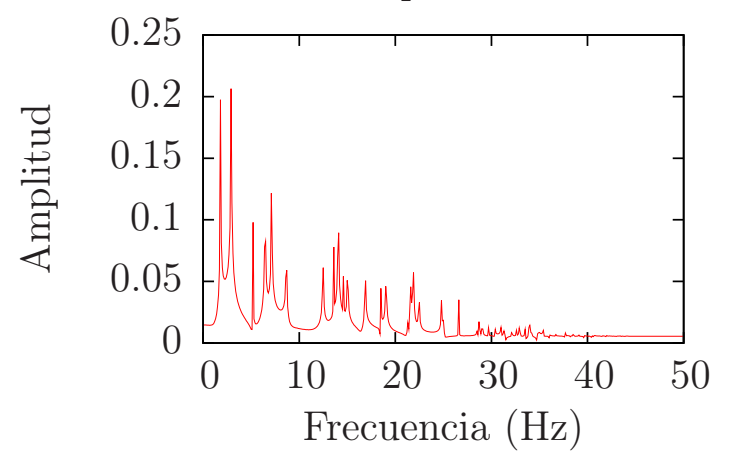

Figura 4.8: Excitación y respuesta en dos puntos de una placa [0] simplemente apoyada en los dominios del tiempo y la frecuencia. Excitación: Pulso gaussiano 
Excitación

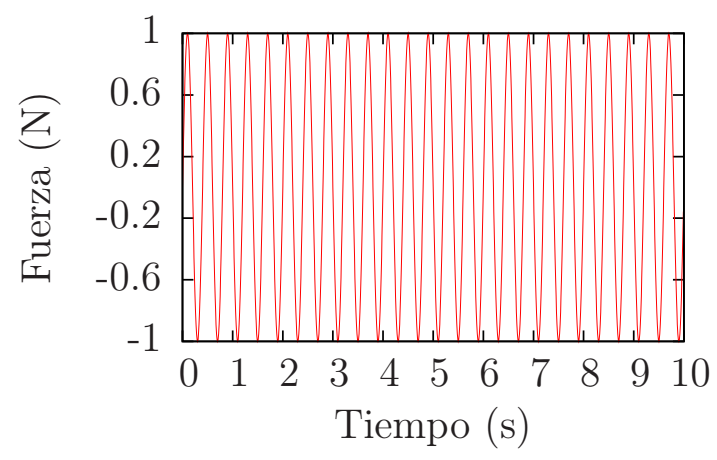

Respuesta 1

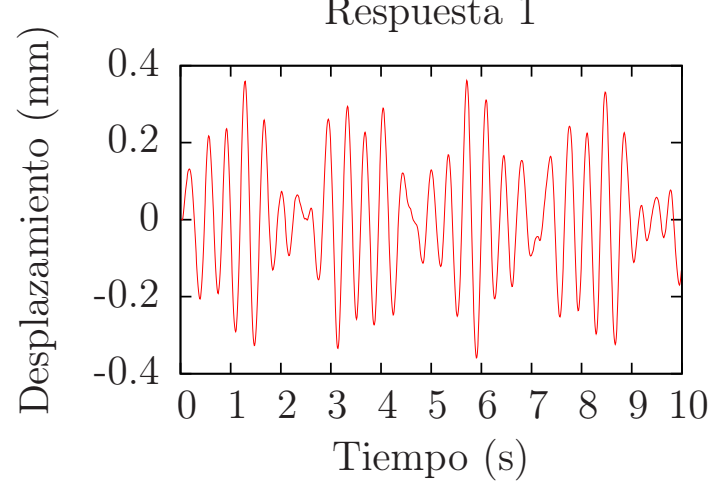

Respuesta 2

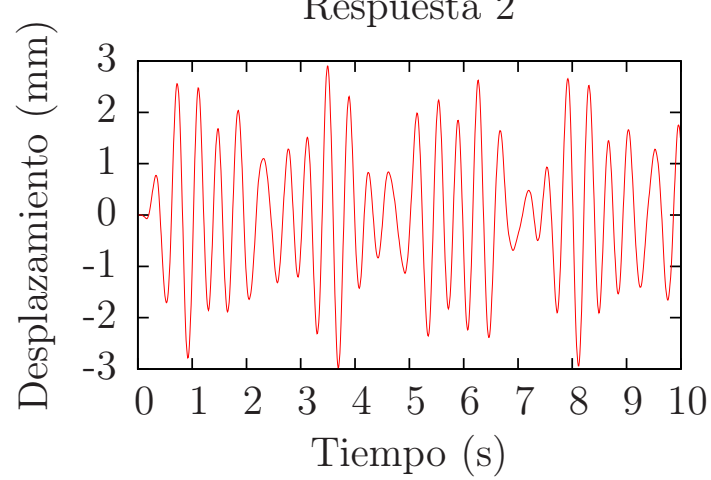

Excitación

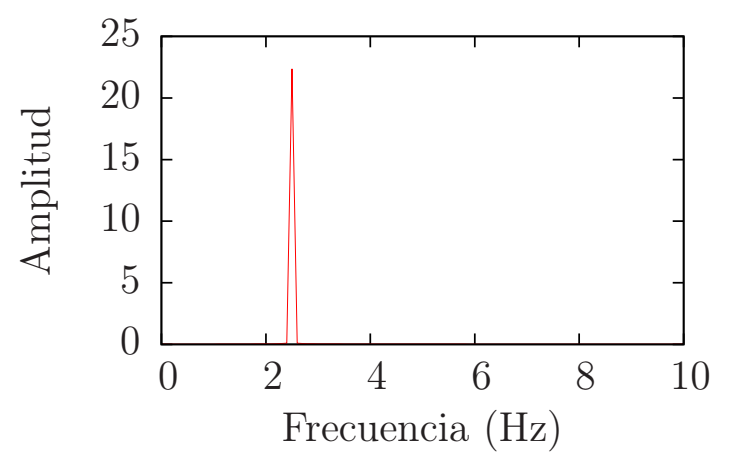

Respuesta 1

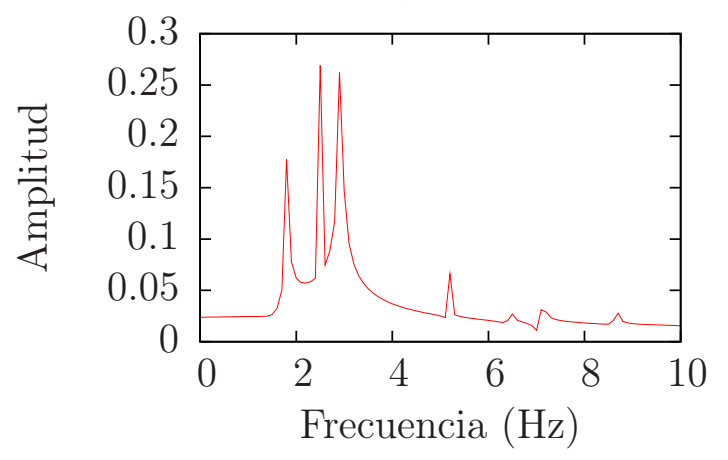

Respuesta 2

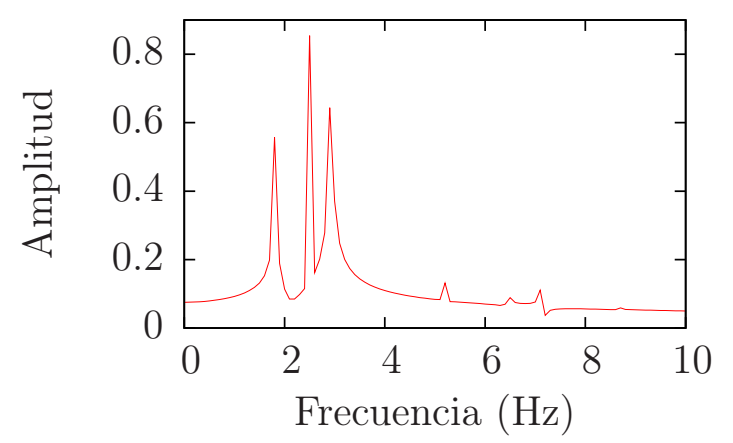

Figura 4.9: Excitación y respuesta en dos puntos de una placa [0] simplemente apoyada en los dominios del tiempo y la frecuencia. Excitación: Seno de $2.5 \mathrm{~Hz}$ 
Excitación

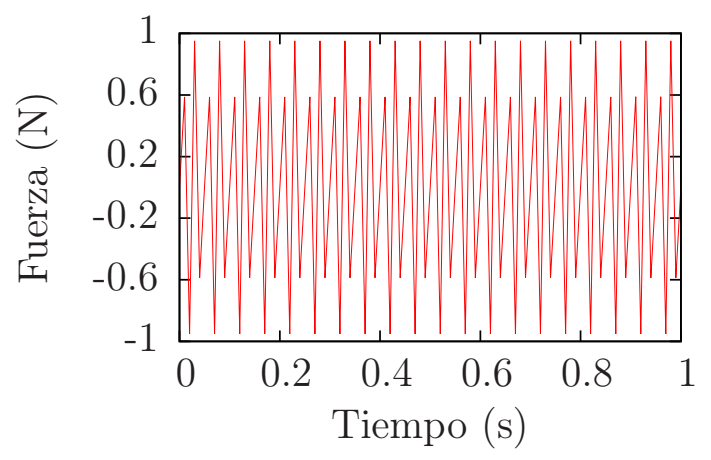

Respuesta 1

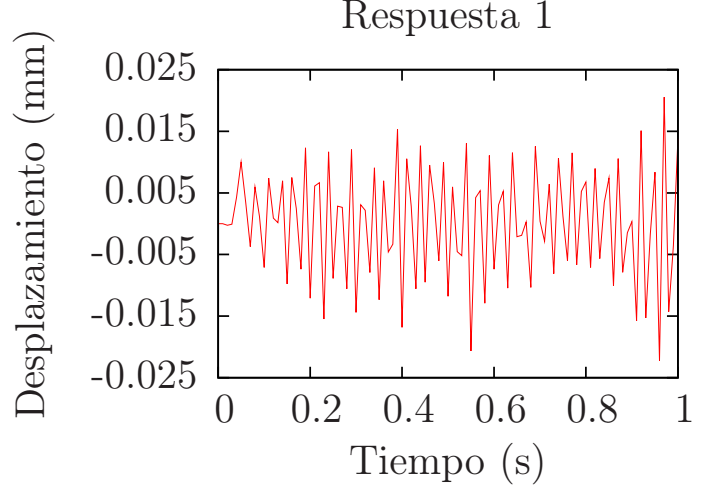

Respuesta 2

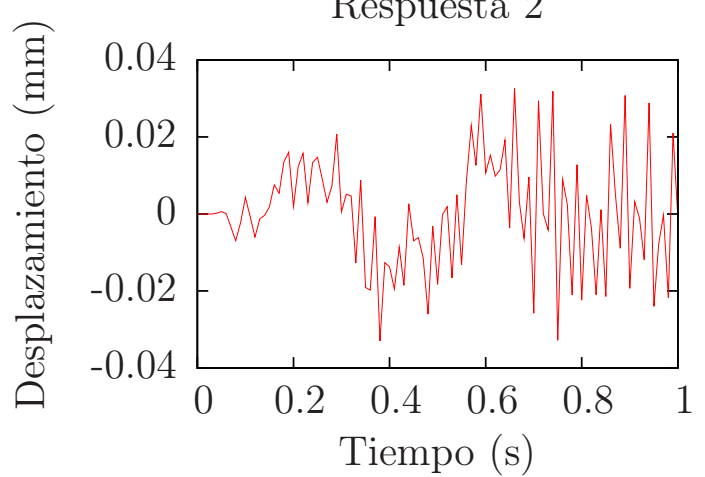

Excitación

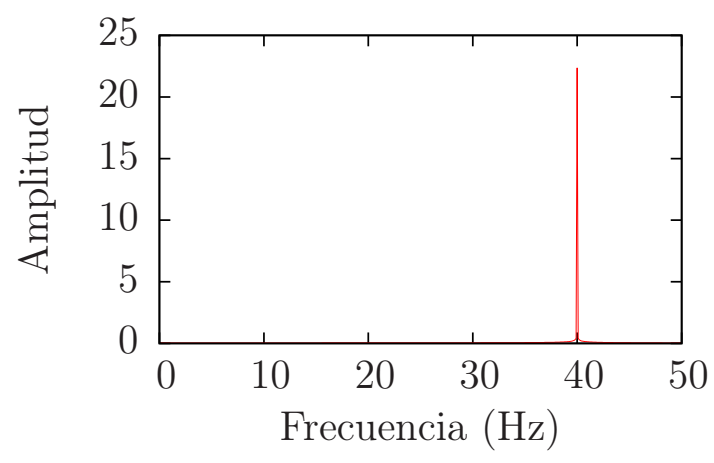

Respuesta 1

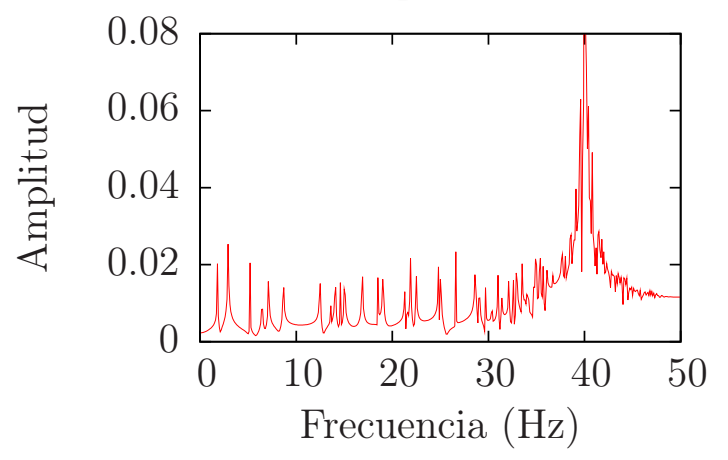

Respuesta 2

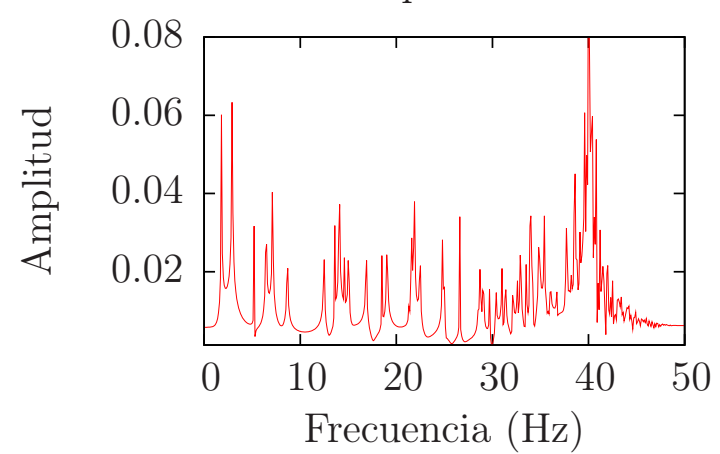

Figura 4.10: Excitación y respuesta en dos puntos de una placa [0] simplemente apoyada en los dominios del tiempo y la frecuencia. Excitación: Seno de $40 \mathrm{~Hz}$ 


\section{Capítulo 5}

\section{Placa multidireccional}

En este capítulo se comparan los resultados obtenidos mediante simulación numérica usando el programa Ansys con los resultados experimentales obtenidos en el proyecto DIDA, citado en el Capítulo 1, en el caso particular de una placa multidireccional de CFRP.

\subsection{Descripción de la placa y el experimento}

Para realizar el experimento se usó una placa fabricada en el Departamento de Materiales y Producción Aeroespacial de la Escuela Técnica Superior de Ingeniería Aeronáutica de la Universidad Politécnica de Madrid. El material usado fué tipo T300/5208 Carbon/Epoxy. La placa estaba compuesta de 16 capas, cada una de ellas con las siguientes propiedades:

\begin{tabular}{|c|c|c|c|c|c|c|c|}
\hline$E_{1}(\mathrm{~Pa})$ & $E_{2}(\mathrm{~Pa})$ & $\nu_{1}$ & $G_{12}(\mathrm{~Pa})$ & $\rho\left(\mathrm{kg} / \mathrm{m}^{3}\right)$ & $h(\mathrm{~m})$ & $a(\mathrm{~m})$ & $b(\mathrm{~m})$ \\
\hline $1.1709 \times 10^{11}$ & $7.7079 \times 10^{9}$ & 0.3076 & $5.388 \times 10^{9}$ & 1900 & $2 \times 10^{-4}$ & 0.501 & 0.501 \\
\hline
\end{tabular}

La secuencia y los ángulos de apilado de la placa fueron:

$$
\text { [-54.3/35.7/-9.34/35.7/-54.3/-54.3/35.7/-9.34/-54.3/35.7] }
$$

Los experimentos se realizaron en el Departamento de Mecánica de Estructuras e Ingeniería Hidráulica de la Universidad de Granada, por investigadores participantes en el proyecto DIDA. La placa se encontraba en posición vertical suspendida de dos hilos ligeros, de forma que se considera con condiciones de contorno libres. Sobre ella se marcaron 85 puntos o nodos donde se colocaron los acelerómetros para medir las aceleraciones. En la Figura 5.1 se puede ver un esquema con los puntos y las dimensiones de la placa.

Con una puntera de acero estandarizada para ensayos de impacto (fabricada por la empresa Brüel \& Kjaer) se golpeó en el nodo 23, realizando un único impacto en cada 


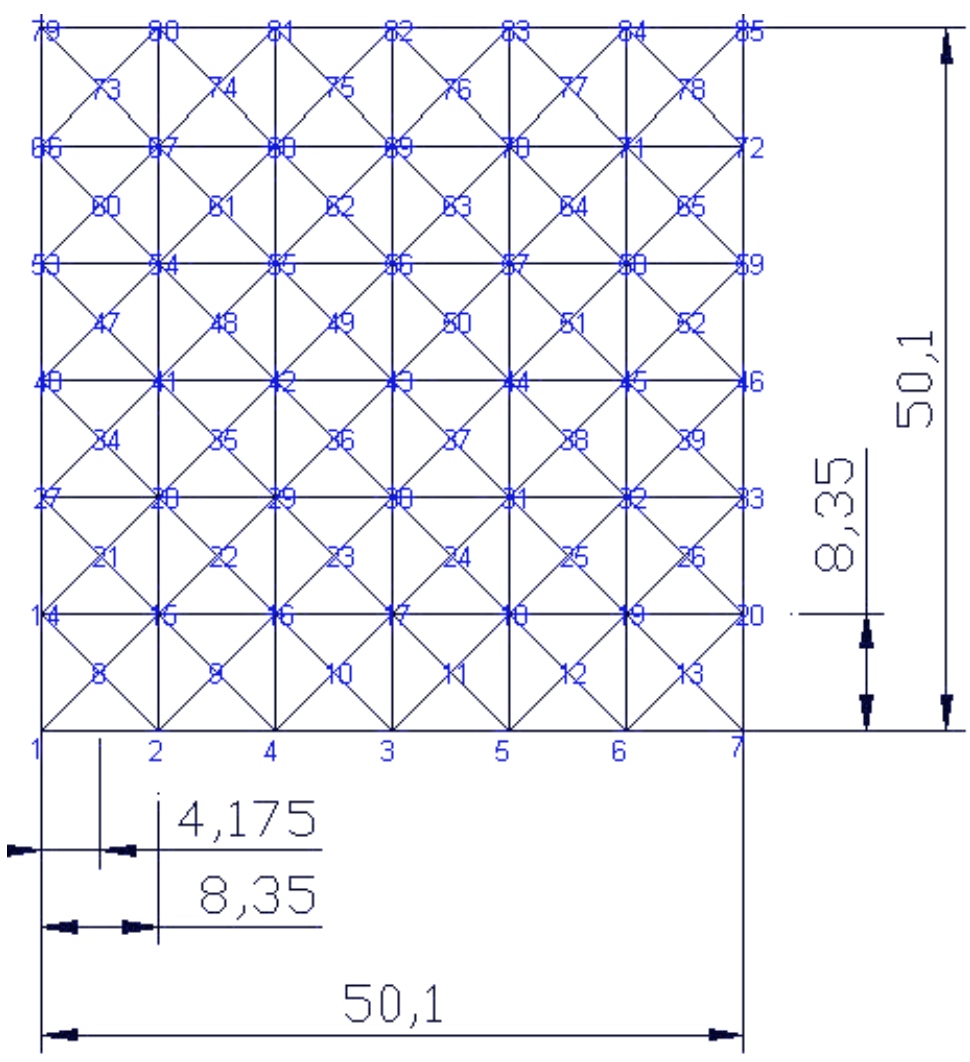

Figura 5.1: Esquema de la disposición de puntos en los que se colocaron los acelerómetros durante el experimento y dimensiones en $\mathrm{cm}$

ensayo. Se utilizaron 7 acelerómetros para medir a la vez la aceleración en 7 puntos, los dos primeros se dejaron fijos en los nodos 8 y 76 y el resto se fueron cambiando hasta medirse las aceleraciones en todas las posiciones, lo que requirió un total de 17 distribuciones de los acelerómetros distintas. En el punto 23, el del impacto, el acelerómetro se colocó por la parte trasera de la placa. Cada distribución se ensayó 5 veces distintas para asegurar la fiabilidad de los resultados obtenidos. En la Tabla 5.1 se especifican las distintas posiciones de los acelerómetros en las distintas configuraciones.

Los acelerómetros usados fueron tipo $4371 \mathrm{~V}$, fabricados por la empresa Brüel \& Kjaer. $\mathrm{Su}$ frecuencia límite superior es de $12.6 \mathrm{KHz}$, la de resonancia $15 \mathrm{KHz}$, su masa es de 11 g y su sensibilidad $9.71 \mathrm{pC} / \mathrm{g}$.

En la Figura 5.2 se muestra una fotografía de la placa durante el experimento en la que se pueden observar, además de la propia placa, los acelerómetros (colocados en la configuración 1), la puntera de acero para el impacto (colocada sobre una barra vertical móvil), los hilos que sujetaban la placa y las marcas de los puntos dónde se colocaron los acelerómetros. 


\begin{tabular}{|c|c|c|c|c|c|c|c|}
\hline Configuración & Acel. 1 & Acel. 2 & Acel. 3 & Acel. 4 & Acel. 5 & Acel. 6 & Acel. 7 \\
\hline 1 & 8 & 76 & 25 & 28 & 37 & 58 & 61 \\
\hline 2 & 8 & 76 & 31 & 34 & 43 & 72 & 67 \\
\hline 3 & 8 & 76 & 10 & 13 & 47 & 39 & 62 \\
\hline 4 & 8 & 76 & 19 & 16 & 38 & 78 & 48 \\
\hline 5 & 8 & 76 & 12 & 32 & 41 & 57 & 66 \\
\hline 6 & 8 & 76 & 18 & 35 & 44 & 65 & 80 \\
\hline 7 & 8 & 76 & 11 & 45 & 55 & 60 & 85 \\
\hline 8 & 8 & 76 & 2 & 26 & 46 & 56 & 73 \\
\hline 9 & 8 & 76 & 4 & 24 & 59 & 54 & 70 \\
\hline 10 & 8 & 76 & 17 & 33 & 50 & 53 & 81 \\
\hline 11 & 8 & 76 & 5 & 22 & 49 & 52 & 69 \\
\hline 12 & 8 & 76 & 3 & 36 & 40 & 74 & 84 \\
\hline 13 & 8 & 76 & 21 & 42 & 68 & 77 & 79 \\
\hline 14 & 8 & 76 & 7 & 9 & 51 & 71 & 75 \\
\hline 15 & 8 & 76 & 15 & 27 & 64 & 83 & 6 \\
\hline 16 & 8 & 76 & 1 & 20 & 29 & 63 & 82 \\
\hline 17 & 8 & 76 & 30 & 14 & 23 & & \\
\hline
\end{tabular}

Tabla 5.1: Disposición de los acelerómetros en cada una de las 17 configuraciones

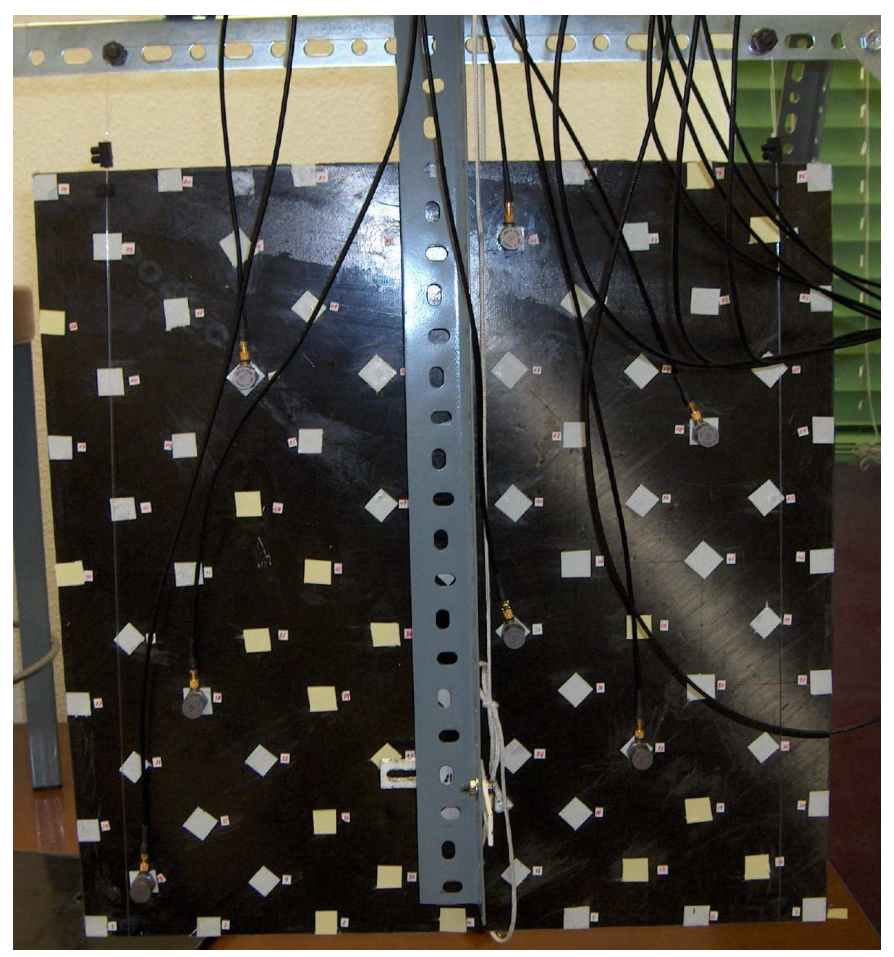

Figura 5.2: Fotografía de la placa durante el experimento. Los acelerómetros se encuentran en la configuración 1 


\subsection{Resultados}

\subsubsection{Señales de vibración}

En total en cada uno de los cinco ensayos se midieron y registraron 117 señales de vibración (aceleración en función del tiempo). Llamaremos $O_{i}^{j}(t)$ a cada una de dichas señales, donde $i$ indica el nodo donde estaba el acelerómetro y $j$ indica el número de ensayo $(j=1, \ldots, 5)$. En la Figura 5.3 se presenta, a modo de ejemplo, la señal temporal registrada en el nodo 53 durante el ensayo $1\left(O_{53}^{1}\right)$, y su Transformada de Fourier.

Como puede verse se trata de señales altamente oscilantes (con un rico contenido en frecuencias), cuya amplitud decae experimentalmente tras el impacto, a medida que la placa va dejando de vibrar por amortiguamiento.

En su Transformada de Fourier se puede observar en primer lugar la aparición de un pico de muy baja frecuencia (en torno a 2 o $3 \mathrm{~Hz}$ ), que correponde con la oscilación global de la placa (movimiento de sólido rígido) y no a las vibraciones naturales de la misma.

La primera frecuencia propia de vibración aparece en un pico muy energético entorno a 39 Hz. A partir de ahí, aparecen numerosos picos (unos más energéticos que otros) indicando las diferentes frecuencias propias de vibración de la placa. Nótese que la amplitud de cada pico depende del punto donde se midió la respuesta vibratoria. Si ese punto está cercano a un nodo del modo de vibración correspondiente, la amplitud será baja. Por el contario, si está cercano a un vientre, la amplitud será alta. Incluso puede ocurrir que que el punto de medida coincida con con un nodo, en cuyo caso dicha frecuencia propia no aparecería en la señal medida en dicho punto.

\subsubsection{Frecuencias y modos propios}

A partir de la ubicación de los picos en la Transformada de Fourier del cunjunto de señales $O_{i}^{j}(t)$ en cada ensayo, se obtuvieron las frecuencias propias de la placa. El resultado está recogido en la Tabla 5.2, en la que se observa que no hay mucha dispersión entre los 5 ensayos. Se presenta también el valor medio de la frecuencias propias.

En la Tabla 5.3 se comparan estas frecuencias medias con las obtenidas numéricamente con el programa Ansys, usando un mallado cuadrado de 36x36 elementos, y un elemento de la biblioteca estándar de Ansys llamado Shell99.

Como puede verse existe un gran acuerdo entre experimentos y simulación, incluso en modos de orden alto.

Mediante la localización de los picos de la Transformada de Fourier de cada una de las señales $O_{i}^{j}(t)$, así como con estas señales se obtuvieron los modos de vibración para cada 


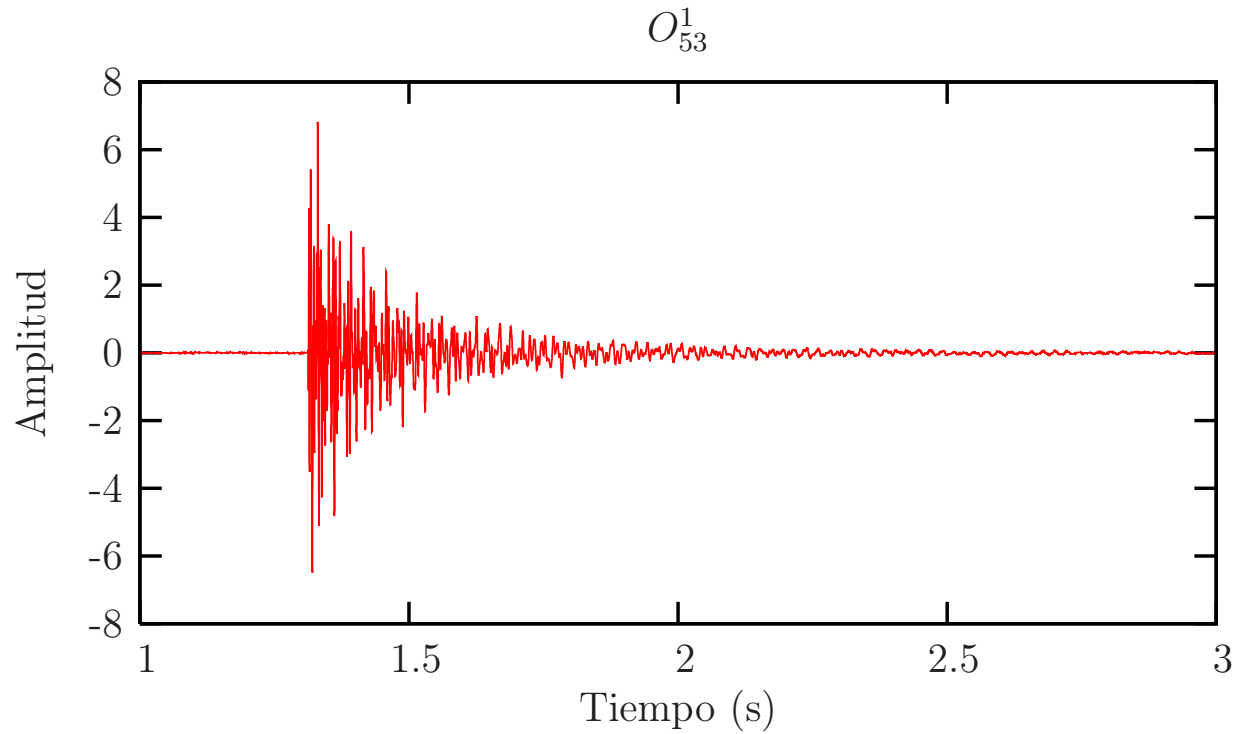

(a)

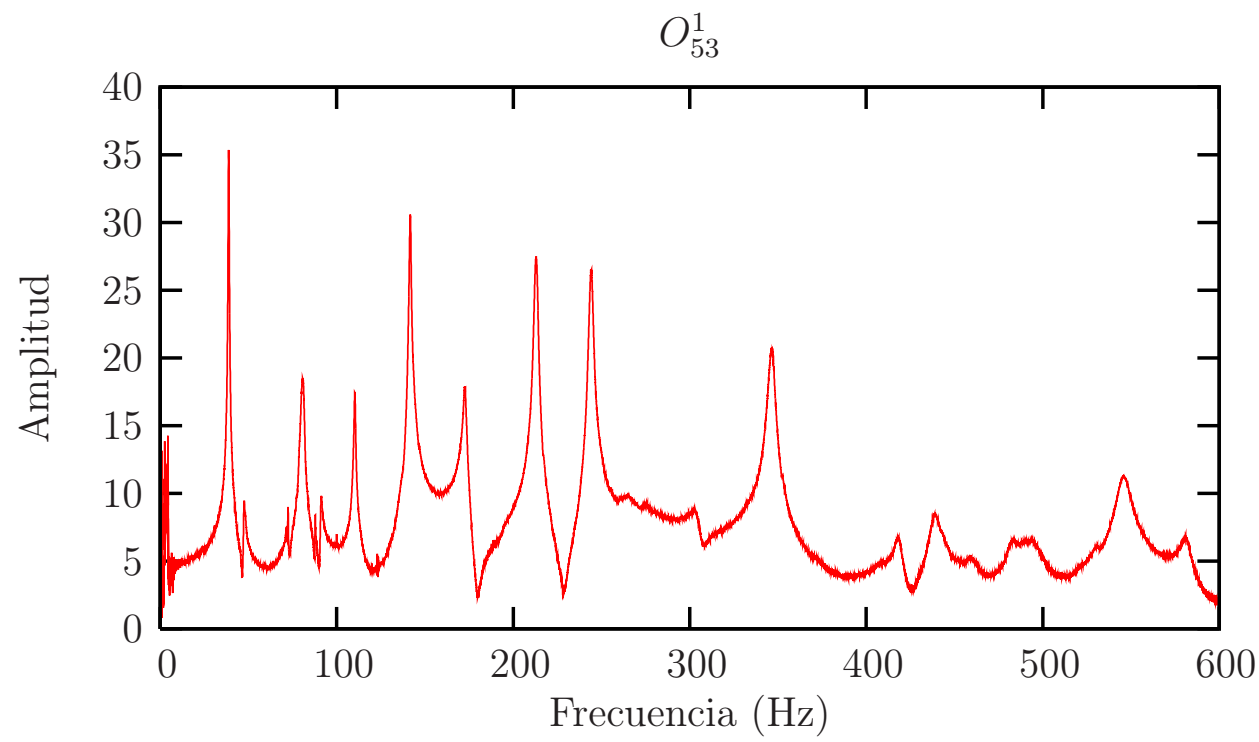

(b)

Figura 5.3: (a) Señal de vibración registrada por el acelerómetro situado en el punto 53 durante el ensayo 1 (b) Transformada de Fourier de dicha señal

uno de los ensayos. Esto se realizó con el programa comercial de análisis modal Artemis, siguiendo el algoritmo descrito en [5].

La media sobre los cinco ensayos, junto a la comparación visual de los modos, nos permitió hacer un identificación de los modos de vibración experimentales, buscando su correspondencia con los modos numéricos obtenidos con Ansys. Los resultados de esta identificación se pueden ver en la Tabla 5.3. 


\begin{tabular}{|c|c|c|c|c|c|c|}
\hline & Ensayo 1 & Ensayo 2 & Ensayo 3 & Ensayo 4 & Ensayo 5 & Media \\
\hline Modo 1 & 39.2 & 39.1 & 38.8 & 39.3 & 39.0 & 39.1 \\
\hline Modo 2 & 47.9 & 48.0 & 48.0 & 48.0 & 48.0 & 48.0 \\
\hline Modo 3 & 79.0 & 79.1 & 79.1 & 78.7 & 78.7 & 78.9 \\
\hline Modo 4 & 92.0 & 92.8 & 91.9 & 90.9 & 91.7 & 91.8 \\
\hline Modo 5 & 141.3 & 141.3 & 141.1 & 141.2 & 141.2 & 141.2 \\
\hline Modo 6 & 171.9 & 171.8 & 171.8 & 171.8 & 171.6 & 171.8 \\
\hline Modo 7 & 187.1 & 188.1 & 186.3 & 188.4 & 187.8 & 187.5 \\
\hline Modo 8 & 196.0 & 196.4 & 196.1 & 194.1 & 194.3 & 195.4 \\
\hline Modo 9 & 213.3 & 213.2 & 213.2 & 213.2 & 213.2 & 213.2 \\
\hline Modo 10 & 242.7 & 243.1 & 241.5 & 243.0 & 240.5 & 242.2 \\
\hline Modo 11 & 271.3 & 269.9 & 273.2 & 271.0 & 271.7 & 271.4 \\
\hline Modo 12 & 313.1 & 313.2 & 314.0 & 314.0 & 314.0 & 313.7 \\
\hline Modo 13 & 349.8 & 350.6 & 350.6 & 350.7 & 346.9 & 349.7 \\
\hline Modo 14 & 351.4 & 352.1 & 351.9 & 352.4 & 350.7 & 351.7 \\
\hline Modo 15 & 420.6 & 421.5 & 422.3 & 422.3 & 422.2 & 421.8 \\
\hline Modo 16 & 434.6 & 434.6 & 434.2 & 434.3 & 430.4 & 433.6 \\
\hline Modo 17 & 552.6 & 553.5 & 554.3 & 552.9 & 553.8 & 553.4 \\
\hline Modo 18 & 612.1 & 615.0 & 611.3 & 611.7 & 616.2 & 613.3 \\
\hline
\end{tabular}

Tabla 5.2: Frecuencias propias de la placa obtenidas en cada uno de los ensayos y su media, en $\mathrm{Hz}$

\begin{tabular}{|c|c|c|}
\hline & Experimental & Numérico \\
\hline Modo 1 & 39.1 & 39.4 \\
\hline Modo 2 & 48.0 & 47.7 \\
\hline Modo 3 & 78.9 & 79.4 \\
\hline Modo 4 & 91.8 & 95.1 \\
\hline Modo 5 & No aparece & 110.6 \\
\hline Modo 6 & 141.2 & 138.4 \\
\hline Modo 7 & 171.8 & 175.3 \\
\hline Modo 8 & 187.5 & 197.7 \\
\hline Modo 8 & 195.4 & 197.7 \\
\hline Modo 9 & 213.2 & 214.6 \\
\hline
\end{tabular}

\begin{tabular}{|c|c|c|}
\hline & Experimental & Numérico \\
\hline Modo 10 & 242.2 & 250.4 \\
\hline Modo 11 & 271.4 & 264.3 \\
\hline Modo 12 & 271.4 & 287.7 \\
\hline Modo 13 & 313.7 & 313.2 \\
\hline Modo 14 & 349.7 & 343.9 \\
\hline Modo 15 & 351.7 & 348.3 \\
\hline Modo 17 & 433.6 & 427.8 \\
\hline Modo 18 & 421.8 & 430.8 \\
\hline Modo 22 & 553.4 & 548.7 \\
\hline Modo 24 & 613.7 & 627.0 \\
\hline
\end{tabular}

Tabla 5.3: Frecuencias propias de la placa obtenidas de forma experimental y numérica, en $\mathrm{Hz}$ 
Seguidamente se presenta la correpondencia entre los modos experimentales y numéricos, desglosando los diferentes casos, siguiendo el orden de la Tabla 5.3.

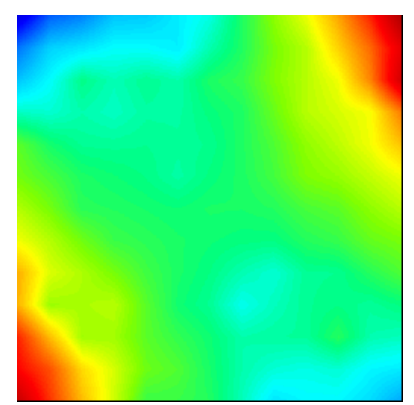

$39.1 \mathrm{~Hz}$

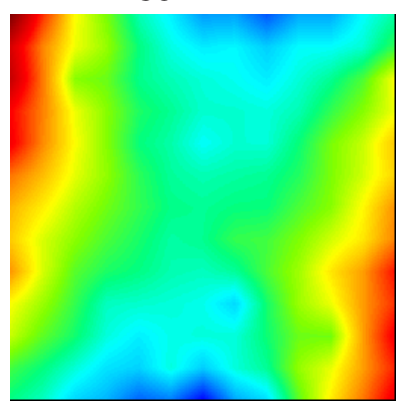

$48.0 \mathrm{~Hz}$

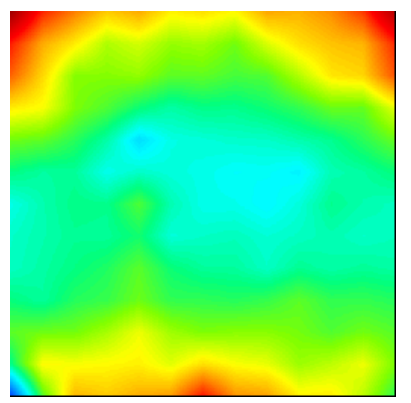

$78.9 \mathrm{~Hz}$

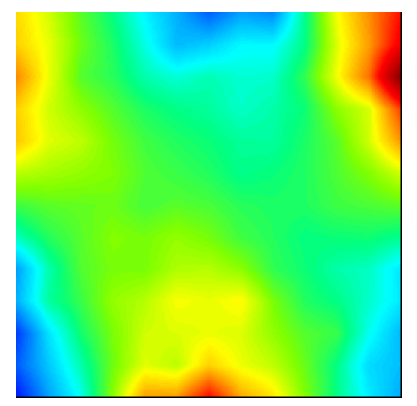

$91.8 \mathrm{~Hz}$

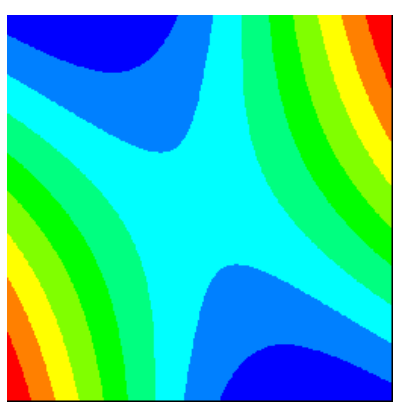

$39.44 \mathrm{~Hz}$

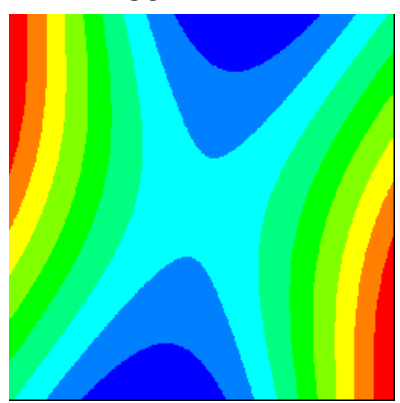

$47.7 \mathrm{~Hz}$

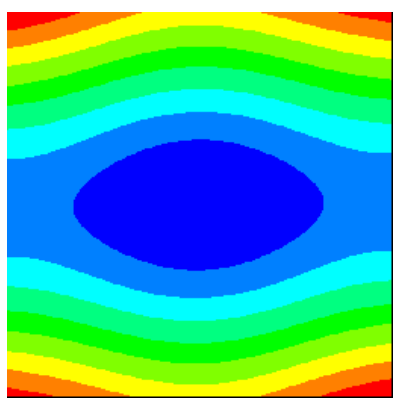

$79.4 \mathrm{~Hz}$

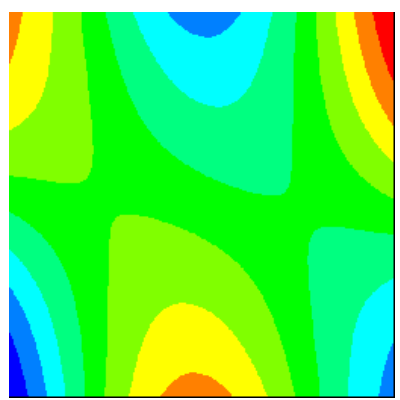

$95.1 \mathrm{~Hz}$

Figura 5.4: Modos de vibración 1, 2, 3 y 4. Izquierda experimental, derecha numérico

Como puede verse en la Figura 5.4 los primeros cuatro modos se ajustan bastante bien, tanto las frecuencias como la forma de los modos. 


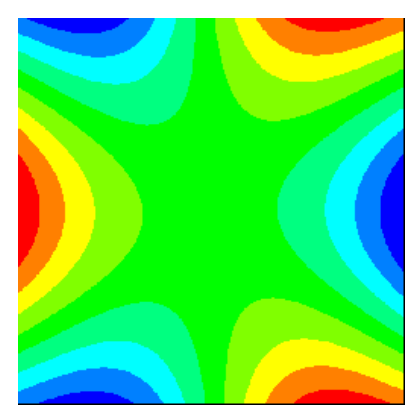

$110.7 \mathrm{~Hz}$

Figura 5.5: Modo de vibración 5, numérico

En la Figura 5.5 se muestra el modo 5, obtenido numéricamente. Este modo no aparece experimentalmente, probablemente porque debido a su forma (movimiento de los bordes) sea difícil de detectar mediante acelerómetros situados dentro de la placa. El hecho de que un modo de orden tan bajo como el quinto no se detecte experimentalmente no es, en principio, un resultado predecible.

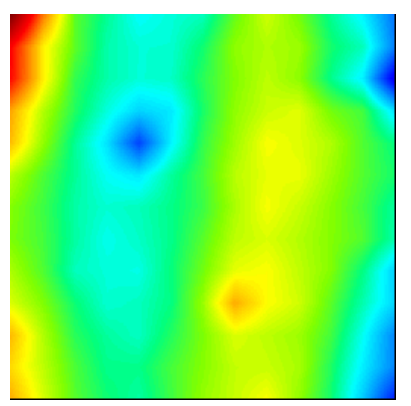

$141.2 \mathrm{~Hz}$

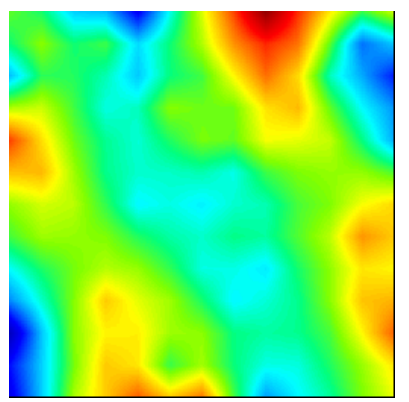

$171.8 \mathrm{~Hz}$

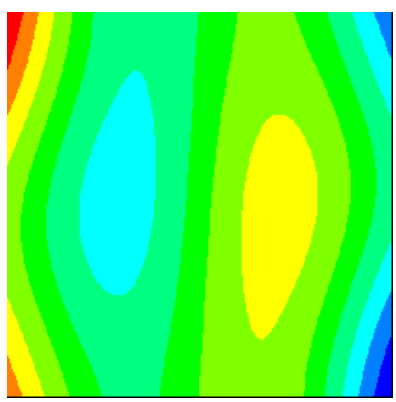

$138.4 \mathrm{~Hz}$

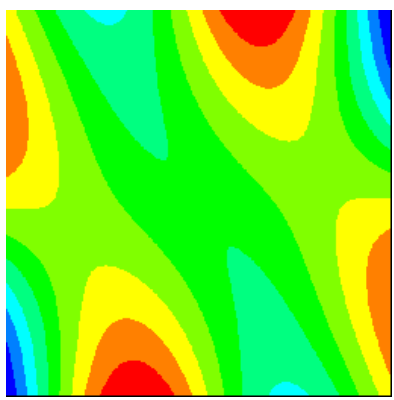

$175.3 \mathrm{~Hz}$

Figura 5.6: Modos de vibración 6 y 7. Izquierda experimental, derecha numérico

En la Figura 5.6 se presentan los modos 6 y 7, que como puede verse se ajustan bastante bien.

Los dos modos experimentales presentados en la Figura 5.7 parecen corresponder al mismo modo numérico. Viendo las frecuencias, es probable que el primero de ellos sea 
espúreo, y sea debido a un error experimental o a una falsa deteccion de pico.

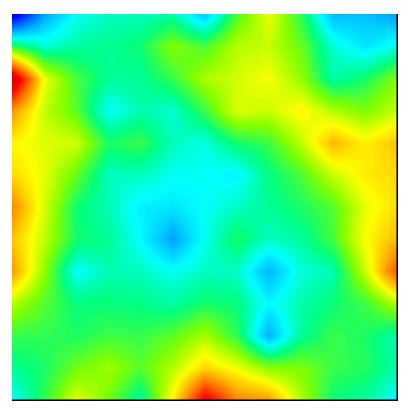

$187.5 \mathrm{~Hz}$

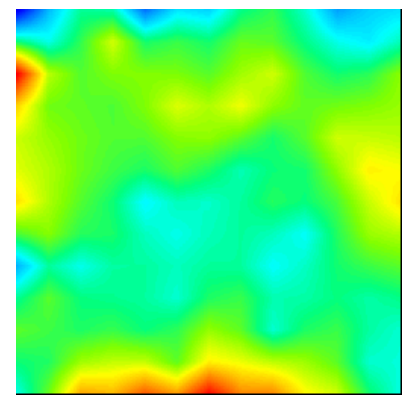

$195.4 \mathrm{~Hz}$

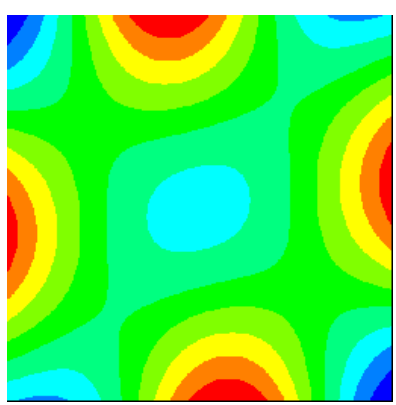

$197.7 \mathrm{~Hz}$

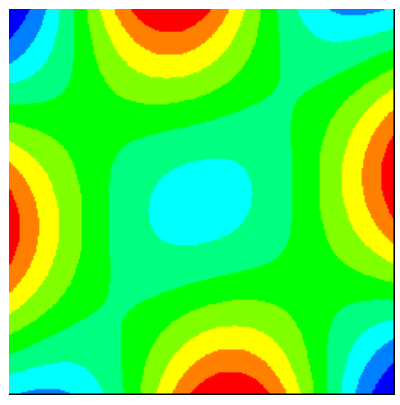

$197.7 \mathrm{~Hz}$

Figura 5.7: Modo de vibración 8. Izquierda experimental, derecha numérico

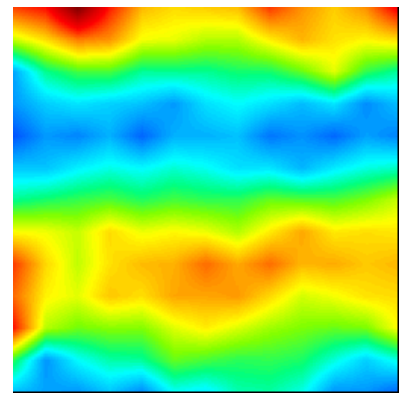

$213.2 \mathrm{~Hz}$

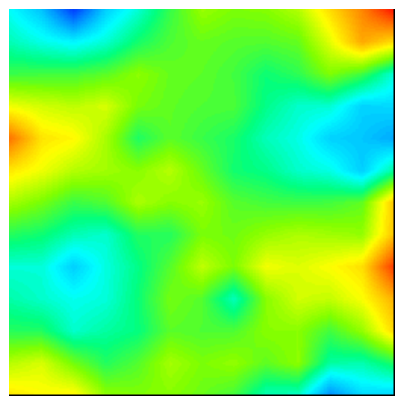

$242.2 \mathrm{~Hz}$

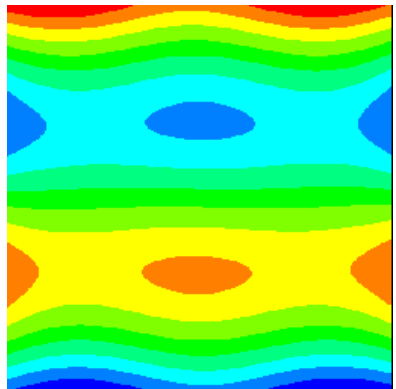

$214.6 \mathrm{~Hz}$

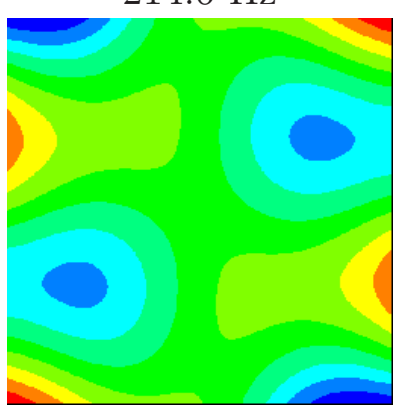

$250.4 \mathrm{~Hz}$

Figura 5.8: Modos de vibración 9 y 10. Izquierda experimental, derecha numérico 
En la Figura 5.8 se muestran los modos 9 y 10, que nuevamente se ajustan bastante bien.

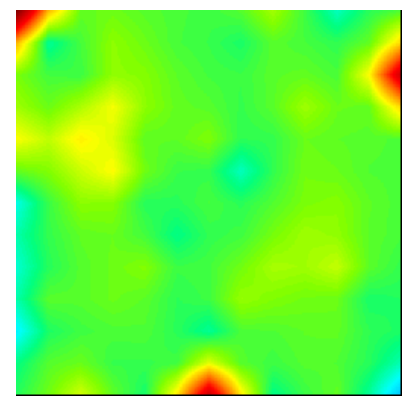

$271.4 \mathrm{~Hz}$

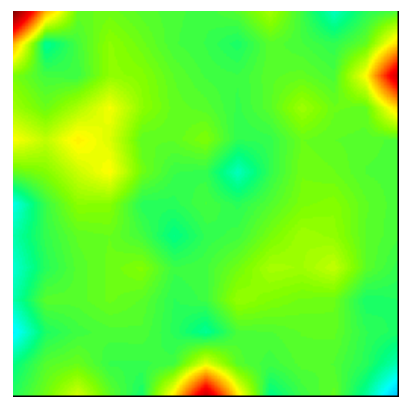

$271.4 \mathrm{~Hz}$

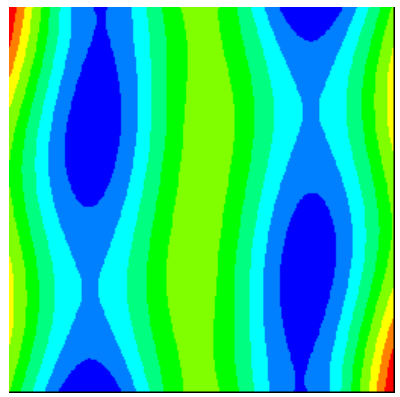

$264.3 \mathrm{~Hz}$

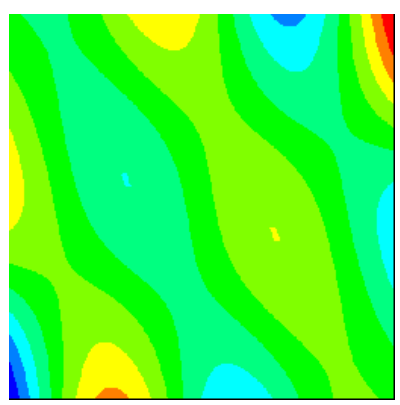

$287.7 \mathrm{~Hz}$

Figura 5.9: Modos de vibración 11 y 12. Izquierda experimental, derecha numérico

En la Figura 5.9 se muestra un modo experimental que no aparece numéricamente. A cambio aparecen dos modos numéricos, por lo que es probable que este modo experimental corresponda realmente a una mezcla de dos modos.

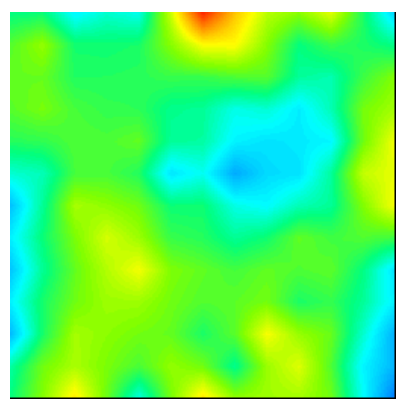

$313.7 \mathrm{~Hz}$

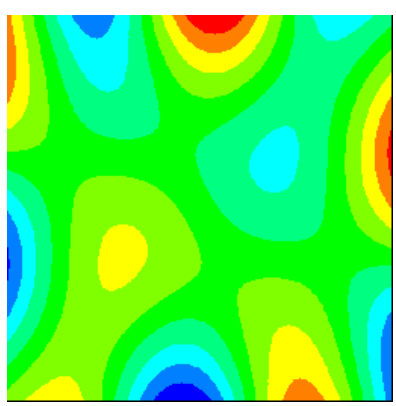

$313.2 \mathrm{~Hz}$

Figura 5.10: Modo de vibración 13. Izquierda experimental, derecha numérico

El modo 13, que se puede ver en la Figura 5.10, presenta un buen ajuste entre experimento y simulación.

En la Figura 5.11 se muestran los modos 14 y 15. Como vemos no se ajustan del todo bien. Quizás sean mezcla de los dos modos numéricos, con una mayor componente del 


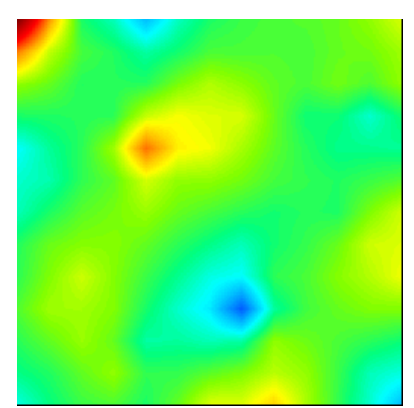

$349.7 \mathrm{~Hz}$

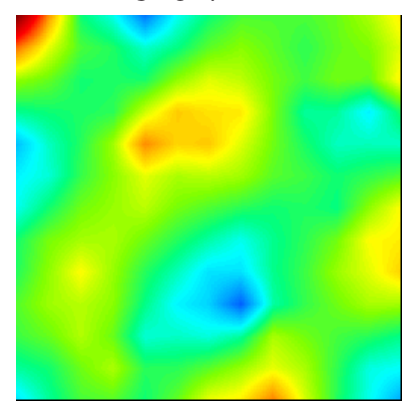

$351.7 \mathrm{~Hz}$

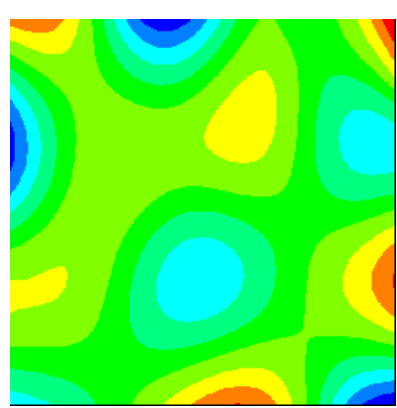

$343.9 \mathrm{~Hz}$

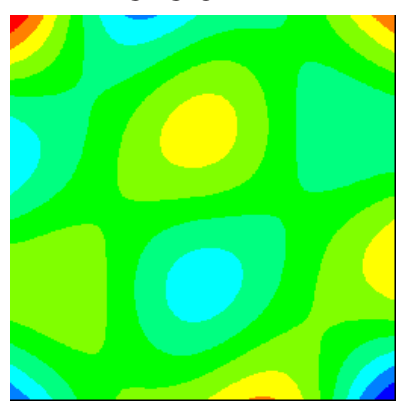

$348.3 \mathrm{~Hz}$

Figura 5.11: Modos de vibración 14 y 15. Izquierda experimental, derecha numérico

segundo, si bien es difícil de decir ya que los dos son parecidos y sus frecuencias son muy próximas.

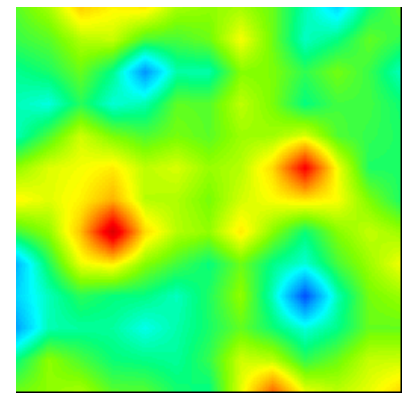

$421.8 \mathrm{~Hz}$

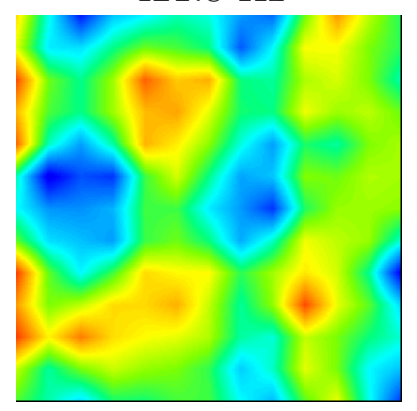

$433.6 \mathrm{~Hz}$

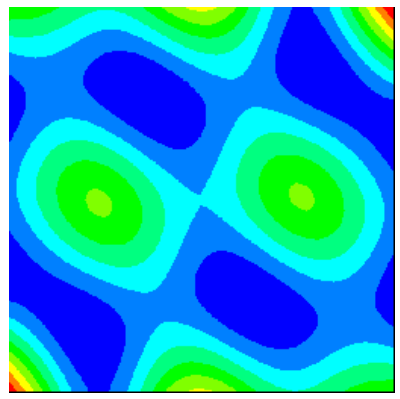

$430.8 \mathrm{~Hz}$

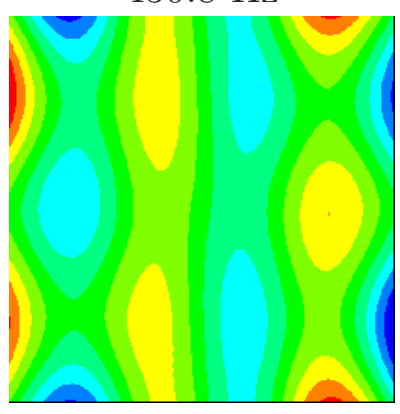

$427.8 \mathrm{~Hz}$

Figura 5.12: Modos de vibración 17 y 18. Izquierda experimental, derecha numérico 
En la Figura 5.12 aparecen los modos 17 y 18. Ocurre lo mismo que para los dos modos anteriores.

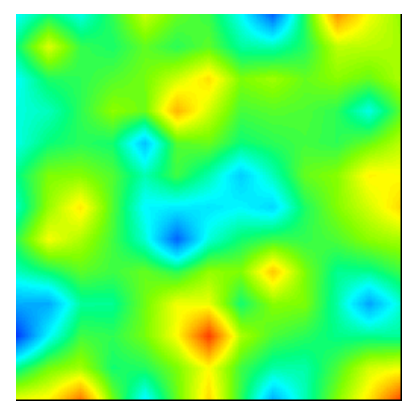

$553.4 \mathrm{~Hz}$

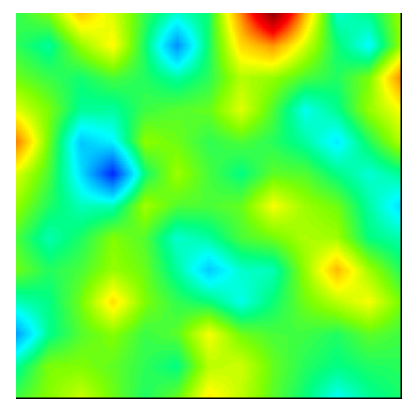

$613.3 \mathrm{~Hz}$

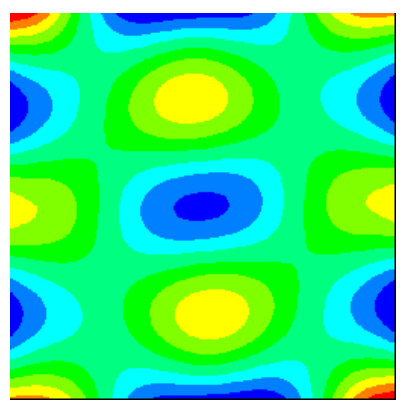

$548.7 \mathrm{~Hz}$

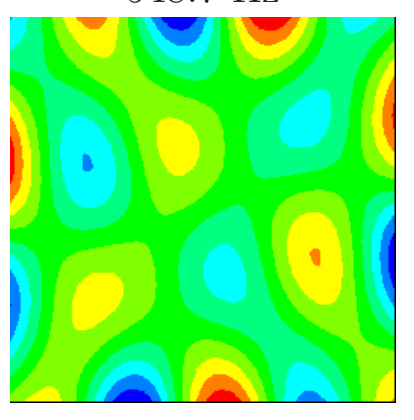

$627.0 \mathrm{~Hz}$

Figura 5.13: Modos de vibración 22 y 24. Izquierda experimental, derecha numérico

Por último, en la Figura 5.13 se muestran los dos últimos modos detectados experimentalmente. Como puede verse parecen ajustarse bastante bien, si bien a órdenes tan altos el ajuste es lógicamente más impreciso. 


\section{Bibliografía}

[1] J.L.P. Aparicio. An integrated micro-macromechanics analysis of progressive failure in cross-ply composite laminates. PhD thesis, Department of Mechanical Engineering, Stanford University, 1992.

[2] J. E. Ashton and J. D. Anderson. The natural modes of vibration of boron-epoxy plates. Shock and Vibration Bulletin, 39(4):81-91, 1969.

[3] F.K. Chang, editor. Structural Health Monitoring 2000, Proceedings of the 2nd Int. Workshop on SHM, Stanford, 1999. Tecnomic Publ. Co.

[4] F.K. Chang, J.L. Perez, and K.Y. Chang. Analysis of thick laminated composites. Journal of Composite Materials, 24(8):801-822, 1990.

[5] D. J. Ewins. Modal Testing: Theory, Practice and Application (Mechanical Engineering Research Studies Engineering Design Series). Taylor \& Francis Group, 2003.

[6] C.P. Fritzen, G. Mengelkamp, and A. Guemes. A cfrp plate with piezo-electric actuators and sensors as self-diagnosing intelligent structure. In Proceedings of the International Conference on Noise and Vibration Engineering (ISMA 2002), pages 185-191, 2002.

[7] T. J. R. Hughes. The Finite Element Method: Linear Static and Dynamic Finite Element Analysis. Prentice-Hall, New Jersey, 1987.

[8] R. M. Jones. Mechanics Of Composite Materials. Taylor \& Francis Group, 1998.

[9] R. Pascual-Jiménez. Model based structural damage assessment using vibrations measurements. PhD thesis, University of Liege, Belgium, 1999.

[10] A. Rytter. Vibration Based Inspection of Civil Engineering Structures. PhD thesis, Aalborg University, Denmark, 1993.

[11] J.M. Whitney. Structural Analysis of Laminated Anisotropic Plates. CRC Press, 1987. 
[12] O. C. Zienkiewicz, R. L. Taylor, and J. Z. Zhu. The Finite Element Method: Its Basis and Fundamentals, volume 1. Elsevier, United Kingdom, 6th edition, 2005. 